\title{
A stochastic multiscale model for acid mediated cancer invasion
}

\author{
Sandesh Hiremath* $^{*} \quad$ Christina Surulescu ${ }^{\dagger}$
}

June 30, 2014

\begin{abstract}
Cancer research is not only a fast growing field involving many branches of science, but also an intricate and diversified field rife with anomalies. One such anomaly is the consistent reliance of cancer cells on glucose metabolism for energy production even in a normoxic environment. Glycolysis is an inefficient pathway for energy production and normally is used during hypoxic conditions. Since cancer cells have a high demand for energy (e.g. for proliferation) it is somehow paradoxical for them to rely on such a mechanism. An emerging conjecture aiming to explain this behavior is that cancer cells preserve this aerobic glycolytic phenotype for its use in invasion and metastasis. We follow this hypothesis and propose a new model for cancer invasion, depending on the dynamics of extra- and intracellular protons, by building upon the existing ones. We incorporate random perturbations in the intracellular proton dynamics to account for uncertainties affecting the cellular machinery. Finally, we address the well-posedness of our setting and use numerical simulations to illustrate the model predictions.
\end{abstract}

\section{Introduction}

A recent approach in cancer therapy is to consider the role of tumor microenvironment in the onset of malignancy in tumors. Gatenby \& Gillies [12] suggested that environmental conditions may drive the selection of the cancerous phenotype. Hypoxia and acidity, for instance, are factors that can trigger the progression from benign to malignant growth [9, 44]. To survive in their environment, tumor cells upregulate certain proton extrusion mechanisms. This boosts apoptosis in normal cells, thereby allowing the neoplastic tissue to extend into the available space. Tumour acidification was recognised as an intrinsic property of both poor vasculature and altered cancer cell metabolism. Moreover, the pH directly influences the metastatic potential of tumor cells 1, 30.

Starting from these facts, Gatenby \& Gawlinski [8] proposed a model for the acid-mediated tumor invasion which uses reaction-diffusion partial differential equations (RD-PDEs) to describe the interaction between the density of normal cells, tumor cells, and the concentration of $\mathrm{H}^{+}$ions produced by the latter. Traveling waves were used in this framework to explain the aggressive action of cancer cells on their surroundings [7]. Further developments of Gatenby \& Gawlinski's model involve both vascular and avascular growth of multicellular tumor spheroids, assuming rotational symmetry, for which existence and qualitative properties of the solutions were investigated [36]. In 28] the model in [8] for acid-mediated tumor invasion was reconsidered, wherein crowding effects (due to competition with cancer cells) in the growth of normal cells was taken into account. The global existence of a unique solution was proved via an iteration argument.

All the models mentioned above consider macroscopic dynamics of cancer and normal cell populations which are coupled - still on the macrolevel - with the evolution of extracellular $\mathrm{H}^{+}$ concentration and possibly also with the concentration of MDEs 29. It is clear, however, that subcellular, microlevel proton dynamics are actually regulating and are influenced significantly by the events on the higher (i.e., macroscopic and mesoscopic) levels [27, 38, 44. Mathematical models studying the interdependence between the activity of several membrane ion transport systems

\footnotetext{
*hiremath@mathematik.uni-kl.de
}

†surulescu@mathematik.uni-kl.de 
and the changes in the peritumoral space were proposed by Webb et al. [46, 45. They also involve intracellular proton buffering, effects on the expression/activation of MMPs and proton removal by vasculature. [45] also accounts for the influence of alkaline intracellular $\mathrm{pH}$ on the growth of tumor cells. Such a model can thus be seen as a first step towards a multiscale setting. The actual invasive behavior, however, can only be assessed when spatial dependence is taken into account. This requires a higher dimensional and more complex modeling framework that couples the subcellular dynamics at microscopic scale with the population dynamics at the macroscopic scale. Micro-macro models of this type (in a different, but related context) were proposed and analysed e.g., in [32, 31, 37].

Stochasticity is a relevant feature inherent to many biological processes occurring on all modeling levels. In particular, it seems to greatly influence subcellular dynamics and individual cell behavior. Models taking this into account were proposed and analysed in various contexts (cell dispersal, intracellular signaling, radio-oncological treatment) in [39, 40, 41]. In the framework of acid-mediated tumor invasion, too, experiments suggest stochasticity in $\mathrm{pH}$ dynamics; this refers to variations and uncertainties (essentially due to a random environment) in the behavior of each cell even though they all follow the same biochemical mechanisms. The distribution of intracellular $\mathrm{pH}\left(\mathrm{pH}_{i}\right)$ at any value of extracellular $\mathrm{pH}\left(\mathrm{pH}_{e}\right)$ was found to be broader than what was predicted by theoretical models based on machine noise and stochastic variation in the activity of membrane-based mechanisms regulating $\mathrm{pH}_{i}$ [27. Moreover, excess current fluctuations have been observed in the gating of the ion channels [19].

Motivated by these facts we propose here a stochastic multiscale model for cancer invasion, to be developed in Section 2 and analysed w.r.t. well posedness in Section 3 . Further, in Section 4 we perform some simulation results to illustrate its performance and eventually discuss in Section 5 the results and comment on the potential of this new model class.

\section{Model set-up}

In this section we set up a phenomenological model for the acid mediated tumor invasion. To this aim we indentify four main quantities and account for their dynamics: $H$ denotes the proton concentration and refers only to cancer cells, as we are interested in the effect of tumor-induced acidity. Thereby, we take into account both the intracellular protons (whose concentration we denote with $H_{i}$ ) and the extracellular ones, having concentration $H_{e}$. The other two quantities are the tumor cell density $C$ and the normal cell density $N$.

\subsection{Microscopic dynamics: the intracellular proton concentration}

The dynamics of intracellular protons is described by the following random differential equation:

$$
\partial_{t} H_{i}=-T_{1}\left(H_{i}, H_{e}\right)-T_{2}\left(H_{i}, H_{e}\right)+T_{3}\left(H_{i}\right)+S_{1}(v)-Q\left(H_{i}\right)+F\left(\chi_{t}, H_{i}\right)
$$

$T_{1}, T_{2}$, and $T_{3}$ are real valued functions representing NDCBE, NHE, and AE transporters, respectively. 1 To acquire a concrete form for these transporter terms - in the absence of numerical data - we followed e.g., [46] and tried to mimic for $T_{1}$ and $T_{2}$ functions the qualitative curves obtained experimentally in [4] for the efflux of protons by NDCBE and NHE in MGU-1 cell lines. For the $T_{3}$ function we adopted the approach in [46] and made it a monotone decreasing function of $H_{i}$, since the AE acts as a counter-mechanism for the alkalinization of cytoplasm. Furthermore, $Q$ denotes the function representing the loss of free protons due to intracellular buffering (e.g., by organelles). The function $S_{1}$ in (1) represents the observed constant acid production rate in cancer cells due to aerobic glycolysis. It is parameterized by tissue vasculature $(v)$. The qualitative features of all these functions are depicted in Figure 1 and Figure 2.

As a cell is a complex machinery influenced by a plethora of biochemical and background processes, a phenomenological deterministic model is prone to be highly idealized and fails to account for the complex behavior of the intracellular environment and its interactions with the cell's surroundings. One approach of remove this drawback would be to use random terms serving as an

\footnotetext{
${ }^{1} \mathrm{NDCBE}\left(\mathrm{Na}^{+}\right.$dependent $\mathrm{Cl}^{-}-\mathrm{HCO}_{3}^{-}$exchanger $), \mathrm{NHE}\left(\mathrm{Na}^{+}\right.$and $H^{+}$exchanger $)$and $\mathrm{AE}\left(\mathrm{Cl}^{-}-\mathrm{HCO}_{3}^{-}\right.$or anion exchanger) are specific transporters present on the cell membrane.
} 
Figure 1: Functions representing NDCBE and NHE transporters
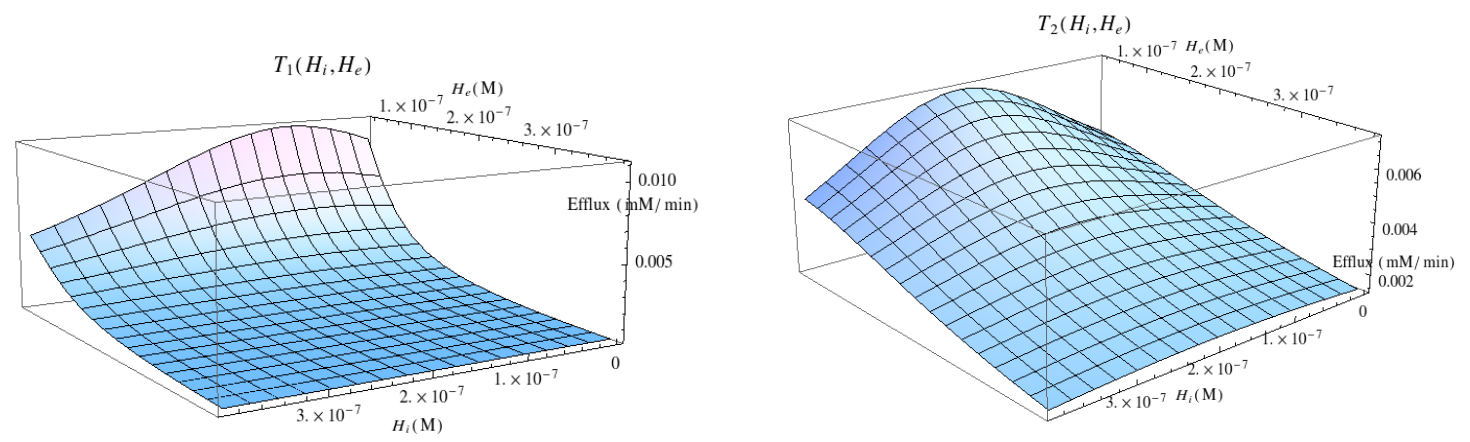

Figure 2: Functions representing AE transporter and proton production and loss terms
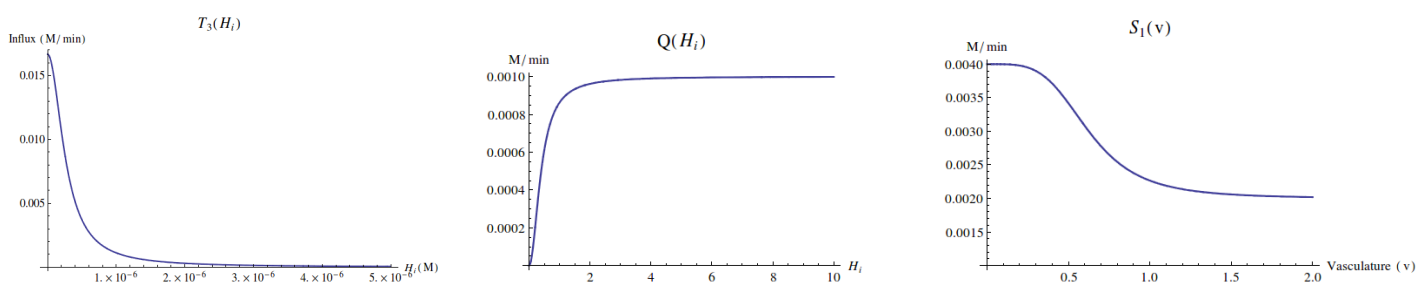

ensemble of uncertainties influencing the proton dynamics. Here we consider a state dependent noise of the following form $2^{2}$

$$
F\left(\chi_{t}, H_{i}\right):=F_{1}\left(H_{i}\right) F_{2}\left(B_{t}^{a, b}\right):=\vartheta H_{i} B_{t}^{a, b},
$$

where $B_{t}^{a, b}$ is a Bownian bridge process starting at $a$ and ending at $b$. Thereby, $a, b \in \mathbb{R}$ and $\vartheta$ are some independent parameters.

\subsection{Extracellular proton concentration}

The next quantity of interest in our model is the extracellular proton concentration $H_{e}(t, x)$, which is modeled to satisfy the following equation:

$$
\partial_{t} H_{e}=T_{1}\left(H_{i}, H_{e}\right)+T_{2}\left(H_{i}, H_{e}\right)-T_{3}\left(H_{i}\right)-S_{2}(v) H_{e}+D_{1} \Delta H_{e}
$$

The transport functions $T_{1}, T_{2}$ and $T_{3}$ are as mentioned above. The function $S_{2}$ is used to describe the removal of acid (protons) from the extracellular (interstitial) space by vasculature and takes the form

$$
S_{2}(v):=a_{5} \kappa v .
$$

We include diffusion as a way to describe acidity patterns in the peritumoral region. The parameter $D_{1}>0$ represents the diffusion coefficient of the extracellular protons. To shorten the notation, we denote by $T\left(H_{i}, H_{e}\right)$ the efflux of protons as a combined effect of $T_{1}, T_{2}$, and $T_{3}$ i.e.

$$
T\left(H_{i}, H_{e}\right):=T_{1}\left(H_{i}, H_{e}\right)+T_{2}\left(H_{i}, H_{e}\right)-T_{3}\left(H_{i}\right) .
$$

We collect the parameters involved in the dynamics of $H_{i}$ and $H_{e}$ into the vector $\Xi:=(v, \kappa)^{T}$. Their values are chosen with the aim of achieving the long time behavior of a reverse $\mathrm{pH}$ gradient.

\subsection{Cell dynamics on the macroscale}

Since we want to study the effect of proton concentrations on cancer cell invasion we need to characterise the dynamics of tumor cell density $C(t, x)$ and normal cell density $N(t, x)$. The

\footnotetext{
${ }^{2}$ other choices involving e.g., an Ornstein-Uhlenbeck process or a bounded function of a Brownian motion are conceivable as well
} 
Figure 3: Cancer proliferation function as a function of $H_{i}$.

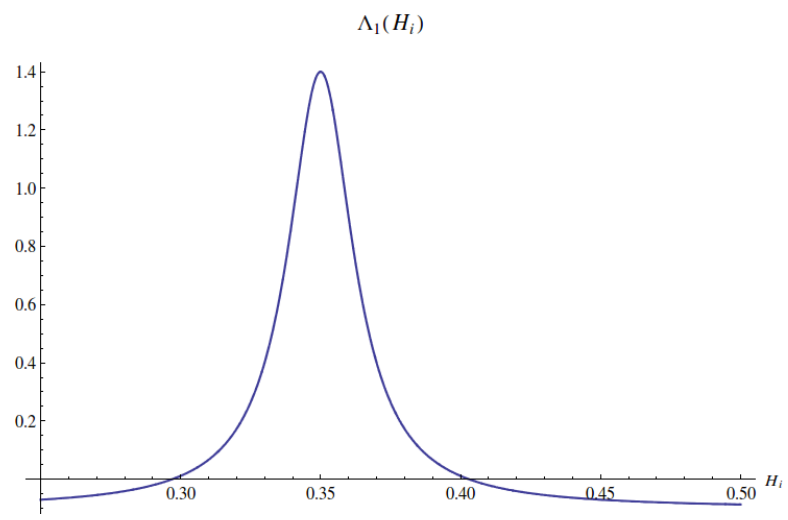

former is supposed to satisfy the reaction-diffusion equation

$$
\partial_{t} C=\omega_{1} \Lambda_{1}\left(H_{i}\right) C\left(1-\eta_{C} \frac{C}{K_{C}}\right)-\omega_{1} \Lambda_{1}^{+}\left(H_{i}\right) \eta_{N} \frac{C N}{K_{N}}+\nabla \cdot\left(D_{2}(C, N) \nabla C\right),
$$

where $\Lambda_{1}^{+}\left(H_{i}\right):=\max \left(\Lambda_{1}\left(H_{i}\right), 0\right)$ is the positive part of $\Lambda_{1}\left(H_{i}\right)$. We use a (modified) LotkaVolterra type reaction term to model inter- and intra-species competition between cancer cells and normal cells. This can be explicitly written as $\omega_{1} C\left(1-\eta_{C} \frac{C}{K_{C}}-\eta_{N} \frac{N}{K_{N}}\right)$. The proliferation function $\Lambda_{1}\left(H_{i}\right)$ represents the influence of intracellular proton concentration on the growth of cancer cells. According to [44, 35, relatively high $p H_{i}$ fosters cell division and provides resistance to cell apoptosis. Hence (see [4]), higher $p H_{i}$ may cause a reentry of the cell into the mitotic phase or suppression of mitotic arrest. These features are incorporated by introducing a rate function (also termed proliferation or switching function) $\Lambda_{1}$ for the damped logistic growth above.

According to [5], low $\mathrm{pH}_{i}$ activates DNase II ${ }^{3}$ which in turn leads to cell apoptosis. However, there seems to be also a positive correlation between (too) high $p H_{i}$ values and cell apoptosis [5]. Thus, though not true in every sense, it is interesting to study the effect of $\Lambda_{1}$ which takes positive values for all but toxic values of $H_{i}$. The form of the function is show in Figure 3 . Since $\Lambda_{1}\left(H_{i}\right) \in \mathbb{R}$, for the negative part of $\Lambda_{1}\left(H_{i}\right)$ the influence of normal cell population is ignored. This means that when $\Lambda_{1}\left(H_{i}\right)$ is negative only intra-specie competition is prevalent, as a result the normal cell density has no influence during decay of cancer density. Consequently, the Lotka-Volterra reaction term is modified as in the equation (6).

Cancer cells can spread through space and start affecting different areas of the tissue. For modeling cancer dispersal within a selected region of tissue we use a nonlinear operator of the form $\nabla$. $\left(D_{2}(C, N) \cdot \nabla\right)$. The diffusion coefficient is chosen to be inversely proportional to the normal cell density and the cancer cell density, since they act as obstacles and impede each other's movement:

$$
D_{2}(C, N):=\frac{\gamma}{1+\frac{C N}{K_{C} K_{N}}} .
$$

To complete the model we still need to describe the evolution of the normal cell density, which is supposed to decay during the tumor invasion. This decay, on the one hand, is directly proportional to the probability of the interaction between the two cell populations and, on the other hand, is accelerated by the increased acidity of the extracellular region. We introduce a decay function $\Lambda_{2}:=\log _{2.15}\left(1+H_{e}\right)$ to capture the influence of extracellular proton concentration. The rate of decay is a monotone function of $H_{e}$ and is chosen in such a way that -qualitatively- the decay is slow and quantitatively $\Lambda_{2}\left(H_{e}\right)>1$, for all $H_{e}>1$.15. In particular, $\Lambda_{2}\left(H_{e}\right)>1$ for $H_{e}=1.2$ hence for $p H_{e}=6.92081$.

The growth term is ignored, since replication or regeneration of normal cells happen on a much

\footnotetext{
${ }^{3}$ DNase stands for DeoxyriboNuclease, an enzyme that causes DNA fragmentation. DNase II is a type of DNase which becomes active in acidic conditions.
} 
larger time scale, thus having nearly zero overall influence on the time scale of interest in this work. Hence, the equation characterising the dynamics of normal cell density takes the form

$$
\partial_{t} N=-\omega_{2} \Lambda_{2}\left(H_{e}\right) \frac{C}{K_{C}} \frac{N}{K_{N}},
$$

where $\omega_{2}$ denotes the death rate of normal cells. A detailed explanation for the choice of the functions and a detailed reasoning for all equations proposed above can be found in [15].

Now let $I=(0, T] \subset \mathbb{R}^{+}$be a finite time interval and $\Omega \subset \mathbb{R}^{d}, d \in\{1,2,3\}$ be an open bounded spatial domain. Furthermore, let $(\Sigma, \mathcal{A}, \mathbb{P})$ be a complete probability space and let $\chi: I \times \Sigma \rightarrow$ $\mathcal{S}:=\mathbb{R}$ be a $\mathbb{P}$-a.s. continuous real-valued stochastic process. By $\mathcal{A}_{t}$ we denote the filtration generated by the processes $\chi_{t}$ and $\mathfrak{N}$ denotes the system of all $\mathbb{P}$-nullsets that are $\mathcal{A}_{0}$ measurable.

Then using the dimensionless identities in (94) and (95) and dropping the overhead lines therein we get for each $\varsigma \in \Sigma \backslash \mathfrak{N}$ the following non-dimensionalized system:

$$
\begin{gathered}
(\mathrm{SPDM})\left\{\begin{array}{l}
\partial_{t} H_{i}(\varsigma)=-T\left(H_{i}, H_{e}\right)+S_{1}-Q\left(H_{i}\right)+F\left(\chi_{t}(\varsigma), H_{i}\right) \text { in } I \times \Omega \\
H_{i}(0, \varsigma)=H_{i, 0}(\varsigma) \text { in } \Omega \\
\partial_{t} H_{e}(\varsigma)=T\left(H_{i}, H_{e}\right)-S_{2} H_{e}+\Delta H_{e} \quad \text { in } I \times \Omega \\
H_{e}(0, \varsigma)=H_{e, 0}(\varsigma), \quad \text { in } \Omega \\
\nabla H_{e}(\varsigma) \cdot \hat{n}=0 \quad \text { in } \in I \times \partial \Omega
\end{array}\right. \\
(\mathrm{CPDM})\left\{\begin{array}{l}
\partial_{t} C(\varsigma)=\omega_{1} \Lambda_{1}\left(H_{i}\right) C\left(1-\eta_{C} \frac{C}{K_{C}}\right)-\omega_{1} \Lambda_{1}^{+}\left(H_{i}\right) \eta_{N} \frac{C N}{K_{N}} \\
+\nabla \cdot(D(C, N) \nabla C) \quad \text { in } I \times \Omega \\
C(0, \varsigma)=C_{0}(\varsigma) \text { in } \Omega \\
\nabla C(\varsigma) \cdot \hat{n}=0 \quad \text { in } I \times \partial \Omega \\
\partial_{t} N(\varsigma)=-\Lambda_{2} \omega_{2} C N \quad \text { in } I \times \Omega \\
N(0, \varsigma)=N_{0}(\varsigma) \text { in } \Omega .
\end{array}\right.
\end{gathered}
$$

Thereby, SPDM stands for stochastic proton dynamics model and CPDM denotes the cell population dynamics model. Due to the presence of the stochastic term in the microscopic equation $8 \mathrm{a}$ and through the coupling with the rest of the equations, the result is a stochastic multiscale model (abbreviated as SMSM). Nonetheless, for fixed $\varsigma \in \Sigma \backslash \mathfrak{N}$ we have a deterministic system of equations, to which the standard theory of ODE and PDE is applicable. Next we prove the well-posedness of our model and finally perform numerical simulations to assess its qualitative behavior.

\section{Analysis of the stochastic multiscale model}

In this chapter we analyze the well posedness of the stochastic multiscale model for acid mediated cancer invasion. The analysis of (SPDM) and (CPDM) can be handled sequentially, since only the latter is coupled with the former and not the other way round. For simplicity of writing we denote

\section{Notation 1.}

$$
\begin{aligned}
R_{1}\left(H_{i}, H_{e}\right) & :=-T\left(H_{i}, H_{e}\right)+S_{1}-Q\left(H_{i}\right), \\
R_{2}\left(H_{i}, H_{e}\right) & :=T\left(H_{i}, H_{e}\right) \\
R_{3}\left(H_{i}, C, N\right) & :=\omega_{1} \Lambda_{1}\left(H_{i}\right) C\left(1-\eta_{C} \frac{C}{K_{C}}\right)-\omega_{1} \Lambda_{1}^{+}\left(H_{i}\right) \eta_{N} \frac{C N}{K_{N}}, \\
R_{4}\left(H_{e}, C, N\right) & :=-\Lambda_{2}\left(H_{e}\right) C N,
\end{aligned}
$$

where $T\left(H_{i}, H_{e}\right)$ is given in equation (5). (For notational convenience the growth rate $\omega_{1}$ and the decay rate $\omega_{2}$ are moved into the function $\Lambda_{1}\left(H_{i}\right)$ and $\Lambda_{2}\left(H_{2}\right)$, respectively.)

\subsection{Preliminaries}

Our first step here is to we refine the probability space to accommodate only a.s. bounded processes. 


\subsubsection{Boundedness of the random component $F_{2}\left(\chi_{t}\right)$ in the noise term $F$ (see (2))}

Definition 1 ( $\varepsilon$-acceptance set). Let $C_{F}>0$ be a fixed constant. The set

$$
\mathcal{G}(\varepsilon):=\left\{\varsigma: \mathbb{P}\left(\sup _{t \in[0, T]}\left|F_{2}\left(\chi_{t}(\varsigma)\right)\right|>C_{F}\right)<\varepsilon\right\}
$$

is defined to be the $\varepsilon$-acceptance set.

Definition 2 ( $\varepsilon$-exception set). The set

$$
\mathcal{E}(\varepsilon):=\Sigma \backslash \mathcal{G}(\varepsilon)
$$

is called the $\varepsilon$-exception set.

Further assume $F_{2}\left(\chi_{t}\right)$ is a semi-martingale, i.e., it can be written as

$$
F_{2}\left(\chi_{t}\right)=A_{t}+M_{t}
$$

where $M_{t}$ is a right continuous martingale and $A_{t}$ is a càdlàg process locally adapted and with bounded variation. By Doob's martingale inequality it follows then that

$$
\mathbb{P}\left(\sup _{t \in[0, T]}\left|F_{2}\left(\chi_{t}\right)\right|>C_{F}\right) \leq \frac{\mathbb{E}\left(M_{T}^{2}\right)}{\tilde{C}_{F}^{2}},
$$

where $\tilde{C}_{F}:=C_{F}-\sup _{t \in[0, T]}\left|A_{t}\right|$.

It is important to note that on the set $\mathcal{G}(\varepsilon), F_{2}\left(\chi_{t}\right) \leq C_{F}$ with the probability $1-\varepsilon$. As a result by choosing $\varepsilon \ll 1$ one can achieve "nearly" $\mathbb{P}$-a.s. boundedness. This motivates us to exclude the exception set, $\mathcal{E}(\varepsilon)$, from $\Sigma$, so that we can obtain a.s. boundedness. Following this idea we define a (sub)probability space $\left(\Sigma_{\varepsilon}, \mathcal{A}_{\varepsilon}, \mathbb{P}_{\varepsilon}\right)$, such that:

1. $\Sigma_{\epsilon}:=\Sigma \backslash \mathcal{E}(\varepsilon)$ is the new event space.

2. $\mathcal{A}_{\varepsilon} \subset \mathcal{A}$ is the corresponding $\sigma$-algebra of $\Sigma_{\varepsilon}$.

3. $\mathbb{P}_{\varepsilon} \ll \mathbb{P}$ is the new probability measure which is absolutely continuous with respect to $\mathbb{P}$. The requirement of absolute continuity ensures the property that all $\mathbb{P}$-null sets are also $\mathbb{P}_{\varepsilon}$-null sets.

This new probability space now contains only those sample paths of the process $\left(F_{2}\left(\chi_{t}\right)\right)_{t \in[0, T]}$ that are $\mathbb{P}_{\varepsilon}$-a.s. bounded. Thus in this sense, we define $C_{F}^{\varepsilon}$ to be the uniform upper bound for the process $\left(F_{2}\left(\chi_{t}\right)\right)_{t \in[0, T]}$.

Lemma 3.1. For a given small exception probability $\varepsilon$ such that $1 \gg \varepsilon>0$ and $T<\infty$, if there exists of a non-empty (sub) probability space $\left(\Sigma_{\varepsilon}, \mathcal{A}_{\varepsilon}, \mathbb{P}_{\varepsilon}\right)$, then there exists a constant $C_{F}^{\varepsilon}<\infty$ such that the semimartingale process $\left(F_{2}\left(\chi_{t}\right)\right)_{t \in[0, T]}$ restricted to $\Sigma_{\varepsilon}$ (denoted as $\left.\left(F_{2}^{\varepsilon}\left(\chi_{t}\right)\right)_{t \in[0, T]}\right)$ is $\mathbb{P}_{\varepsilon}$-a.s. uniformly bounded, such that

$$
F_{2}^{\varepsilon}\left(\chi_{t}(\varsigma)\right) \leq C_{F}^{\varepsilon}, \quad \forall t \in[0, T], \forall \varsigma \in \Sigma_{\varepsilon} .
$$

Proof. Clear from the construction procedure for $\left(\Sigma_{\varepsilon}, \mathcal{A}_{\varepsilon}, \mathbb{P}_{\varepsilon}\right)$ illustrated above.

Remark 1. An immediate consequence is that the noise term $F_{2}\left(\chi_{t}\right):=\vartheta \chi_{t}$, with $\chi_{t}:=B_{t}^{a, b}$ and

$$
B_{t}^{a, b}:=a+(b-a) \frac{t}{T}+W_{t}-W_{T} \frac{t}{T} \quad(t \in[0, T])
$$

is a $\mathbb{P}_{\varepsilon}$-a.s. uniformly bounded process. Indeed, $\left(B_{t}^{a, b}\right)$ is a semimartingale, which can be seen from the following Itô integral definition of a Brownian bridge process

$$
B_{t}^{a, b}:=a+(b-a) \frac{t}{T}+(T-t) \int_{0}^{t} \frac{1}{T-s} d W_{s}=A_{t}+M_{t},
$$


where $M_{t}:=(T-t) \int_{0}^{t} \frac{1}{T-s} d W_{s}$ is a martingale and $A_{t}:=a+(b-a) \frac{t}{T}$ is a deterministic function. Since the Gaussian process $B_{t}^{a, b}$ can be endowed with a complete probability space $(\Sigma, \mathcal{A}, \mathbb{P})$, for any $0<\varepsilon \ll 1$ one can extract a measurable space $\left(\Sigma_{\epsilon}, \mathcal{A}_{e}\right)$ satisfying the above properties and endow it with a probability measure for e.g. $\mathbb{P}_{\varepsilon}:=\mathbb{P} / \mathbb{P}\left(\Sigma_{\epsilon}\right)$ (just a renormalization of $\mathbb{P}$ ). Thus by above lemma the claim holds.

Next we introduce the function spaces in which we expect our solution processes $H_{i}, H_{e}, C$ and $N$ to lie in.

\subsubsection{Function spaces}

Consider $H_{i}(t)$ and $H_{e}(t)$ to be Hilbert space valued processes, i.e., $H_{i}(t, \varsigma)$ and $H_{e}(t, \varsigma)$ are functions in appropriate Hilbert spaces. Moreover, we take the processes themselves to be in some Hilbert space. From this perspective we define the following function spaces:

$$
\begin{aligned}
\mathbb{X} & :=\left(L^{2}\left(\Sigma_{\epsilon} ; \mathfrak{H}^{1,0}\right),\|\cdot\|_{\mathbb{X}}\right) ; \quad\|\mathrm{u}\|_{\mathbb{X}}:=\left(\mathbb{E}_{\varepsilon}\left(\|\mathrm{u}(\varsigma)\|_{\mathfrak{H}^{1,0}}^{2}\right)\right)^{\frac{1}{2}}, \\
\mathbb{Y} & :=\left(L^{2}\left(\Sigma_{\epsilon} ; \mathfrak{H}^{1,1}\right),\|\cdot\|_{\mathbb{Y}}\right) ; \quad \mathbb{Z}:=\left(L^{2}\left(\Sigma_{\epsilon} ; \mathfrak{H}^{1,2}\right),\|\cdot\|_{\mathbb{Z}}\right) \\
\|\mathrm{u}\|_{\mathbb{Y}} & :=\left(\mathbb{E}_{\varepsilon}\left(\|\mathrm{u}(\varsigma)\|_{L^{2}\left(0, T ; H^{1}(\Omega)\right)}^{2}+\left\|\partial_{t} \mathrm{u}(\varsigma)\right\|_{L^{2}\left(0, T ; L^{2}(\Omega)\right)}^{2}\right)\right)^{\frac{1}{2}}, \\
\|\mathrm{u}\|_{\mathbb{Z}} & :=\left(\mathbb{E}_{\varepsilon}\left(\|\mathrm{u}(\varsigma)\|_{L^{2}\left(0, T ; H^{2}(\Omega)\right.}^{2}+\left\|\partial_{t} \mathrm{u}(\varsigma)\right\|_{L^{2}\left(0, T ;\left(H^{1}(\Omega)\right)^{*}\right.}^{2}\right)\right)^{\frac{1}{2}},
\end{aligned}
$$

where

$$
\begin{aligned}
\mathfrak{H}^{1,0} & :=\left\{u \in L^{2}\left(0, T ; L^{2}(\Omega)\right), \partial_{t} u \in L^{2}\left(0, T ; L^{2}(\Omega)\right)\right\}, \\
\mathfrak{H}^{1,1} & :=\left\{u \in L^{2}\left(0, T ; H^{1}(\Omega)\right), \partial_{t} u \in L^{2}\left(0, T ; L^{2}(\Omega)\right)\right\}, \\
\mathfrak{H}^{1,2} & :=\left\{u \in L^{2}\left(0, T ; H^{2}(\Omega)\right), \partial_{t} u \in L^{2}\left(0, T ;\left(H^{1}(\Omega)\right)^{*}\right)\right\} .
\end{aligned}
$$

The Bochner spaces $L^{2}\left(0, T ; L^{2}(\Omega)\right)\left(\right.$ shortly $\left.\mathbb{L}_{\Omega, T}\right), L^{2}\left(0, T ; H^{1}(\Omega)\right), L^{2}\left(0, T ; H^{2}(\Omega)\right)$ and $\left.L^{2}\left(0, T ;\left(H^{1}(\Omega)\right)^{*}\right)\right)$ are all endowed with their respective standard norm. Finally, we also need the space

$$
\mathbb{L}_{\Sigma}:=L^{2}\left(\Sigma_{\varepsilon} ; L^{2}\left(0, T ; L^{2}(\Omega)\right)\right),\|\mathrm{u}\|_{\mathbb{L}_{\Sigma}}:=\left(\mathbb{E}_{\varepsilon}\left(\|\mathrm{u}(\varsigma)\|_{\mathbb{L}_{\Omega, T}}^{2}\right)\right)^{\frac{1}{2}} .
$$

Here $\mathbb{E}_{\varepsilon}$ is the expectation operator on the refined probability space $\left(\Sigma_{\epsilon}, \mathcal{A}_{\varepsilon}, \mathbb{P}_{\varepsilon}\right)$.

We want the solution $H_{i}$ and $N$ to the equations $8 \mathrm{a}$ and $(9 \mathrm{c})$, respectively, to lie in the space $\mathbb{X}$. This means that the processes $\left(H_{i}(t)\right)_{t \in[0, T]}$ and $(N(t))_{t \in[0, T]}$ lie in $L^{2}\left(\Sigma_{\epsilon}\right)$ and take values in $L^{2}(\Omega)$ for each fixed $t \in[0, T]$. Moreover, due to the embedding $\mathfrak{H}^{1,0} \hookrightarrow C\left(0, T ; L^{2}(\Omega)\right), H_{i}(t)$ and $N(t)$ are continuous as functions of $t \in[0, T]$ if $H_{i}(\varsigma) \in \mathfrak{H}^{1,0}$ and $N(\varsigma) \in \mathfrak{H}^{1,0}$. Thus, $H_{i} \in \mathbb{X}$ and $N \in \mathbb{X}$ imply that, for almost all $\varsigma \in \Sigma_{\epsilon}$, the function $H_{i}(t, \varsigma)$ is continuous in $L^{2}(\Omega)$ with respect to $t \in[0, T]$.

Similarly, we want the solutions $H_{e}$ and $C$ to the equations $(8 \mathrm{~b})$ and $(9 \mathrm{~b})$, respectively, to lie in the space $\mathbb{Y}$ and $\mathbb{Z}$ respectively. This means that the processes $\left(H_{e}(t)\right)_{t \in[0, T]}$ and $(C(t))_{t \in[0, T]}$ take values in $H^{1}(\Omega)$ and belong to $L^{2}\left(\Sigma_{\epsilon}\right)$. Moreover, $H_{e}(t)$ and $C(t)$ are weakly differentiable as functions of $t \in[0, T]$, i.e., $H_{e}(\varsigma) \in \mathfrak{H}^{1,1}$ and $C(\varsigma) \in \mathfrak{H}^{1,2}$. Since $\mathfrak{H}^{1,2}, \mathfrak{H}^{1,1} \hookrightarrow C\left([0, T] ; L^{2}(\Omega)\right)$, we notice that $H_{e} \in \mathbb{Y}$ and $C \in \mathbb{Z}$ implies that, for almost all $\varsigma \in \Sigma_{\epsilon}$, the functions $H_{e}(t, \varsigma)$ and $C(t, \varsigma)$ are continuous in $L^{2}(\Omega)$ with respect to $t \in[0, T]$.

\subsection{Analysis of the stochastic proton dynamics model (SPDM)}

Prior to existence and uniqueness theorems, we make the following

\section{Remark 2.}

- The reaction terms in $8 \mathrm{a}$ and $8 \mathrm{~b}$ are uniformly bounded and Lipschitz continuous. 
- From Lemma 3.1 the noise term $F^{\varepsilon}\left(\chi_{t}, H_{i}\right):=H_{i} F_{2}^{\varepsilon}\left(\chi_{t}\right)$ in $(2)$ is Lipschitz continuous.

Moreover, the following result can be easily verified:

Lemma 3.2. For a fixed parameter vector $\Xi$ the reaction terms $R_{1}\left(H_{i}(t, x, \varsigma), H_{e}(t, x, \varsigma)\right)$ and $R_{2}\left(H_{i}(t, x, \varsigma), H_{e}(t, x, \varsigma)\right)$ in (10) and (11) are uniformly bounded for all $t \in[0, T], x \in \Omega, \varsigma \in \Sigma_{\varepsilon}$. Hence for $p \in[1, \infty], R_{1}, R_{2} \in L^{p}\left(\Sigma_{\varepsilon} ; L^{p}([0, T] \times \Omega)\right)$.

The main aim of this section is to show the existence of the weak solution to (SPDM) and prove its uniqueness. The idea is to find the pathwise weak solution of (SPDM), i.e., for each $\varsigma \in \Sigma_{\epsilon}$ to find $H_{i}(\varsigma) \in \mathfrak{H}^{1,0}$ and $H_{e}(\varsigma) \in \mathfrak{H}^{1,1}$. From this perspective, equations 8a and 8b are transformed into the following convenient form:

$$
\begin{gathered}
\partial_{t} H_{e}(t, x, \varsigma)-\Delta H_{e}=-S_{2} H_{e}+R_{2}\left(H_{i}, H_{e}\right) \\
H_{e}(0, x, \varsigma)=H_{e, 0}(x, \varsigma), \text { for each } x \in \Omega, \varsigma \in \Sigma_{\epsilon} \\
\frac{d}{d t} H_{i}(t, x, \varsigma)=R_{1}\left(H_{i}, H_{e}\right)+H_{i} F_{2}\left(\chi_{t}\right), \text { for each } x \in \Omega, \varsigma \in \Sigma_{\epsilon} \\
H_{i}(0, x, \varsigma)=H_{i, 0}(x, \varsigma)
\end{gathered}
$$

In order to prove the uniqueness and existence of the pathwise weak solution we first construct a sequence of solutions and then show that it converges in some appropriate sense. This will occupy the rest of this section.

\subsubsection{Iterative construction of a solution sequence for (SPDM)}

We start constructing a sequence of weak solutions for the equations $8 \mathrm{a}$ and $8 \mathrm{~b}$. First we introduce some abbreviations.

Notation 2. Let $H_{e}^{m} \in\left(H_{e}^{m}\right)_{m \in \mathbb{N}^{*}}$ and $H_{i}^{m} \in\left(H_{i}^{m}\right)_{m \in \mathbb{N}^{*}}$. For the reaction term $R_{1}\left(H_{i}^{m}, H_{e}^{m}\right)$ we use the short notation

$$
R_{1}^{m}:=R_{1}\left(H_{i}^{m}, H_{e}^{m}\right), \quad \forall m \in \mathbb{N}^{*}
$$

and for the reaction term $R_{2}\left(H_{i}^{m}, H_{e}^{m-1}\right)$ we denote

$$
R_{2}^{m}:=R_{2}\left(H_{i}^{m}, H_{e}^{m-1}\right), \quad \forall m \in \mathbb{N}^{*} .
$$

Approximate problem for (SPDM): Let $\left(H_{i}^{m}(\varsigma)\right)_{m \geq 0} \subset \mathfrak{H}^{1,0}$ be such that for each $x \in \Omega$ and $\varsigma \in \Sigma_{\varepsilon}$

$$
\begin{aligned}
\frac{d}{d t} H_{i}^{0}(t) & =R_{1}\left(H_{i, 0}, H_{e, 0}\right)+H_{i}^{0} F_{2}^{\varepsilon}\left(\chi_{t}\right) \\
\frac{d}{d t} H_{i}^{m}(t) & =R_{1}^{m-1}(t)+H_{i}^{m} F_{2}^{\varepsilon}\left(\chi_{t}\right) \quad \text { a.e. } x \in \Omega, m \geq 1 \\
H_{i}^{m}(0, x, \varsigma) & =H_{i, 0}(x, \varsigma), \quad \text { for each } x \in \Omega, \varsigma \in \Sigma_{\epsilon} .
\end{aligned}
$$

Let $\left(H_{e}^{m}(\varsigma)\right)_{m \geq 0} \subset \mathfrak{H}^{1,1}$ be the weak solution of

$$
\begin{aligned}
\partial_{t} H_{e}^{0}-\Delta H_{e}^{0} & =-S_{2} H_{e}^{0}+R_{2}\left(H_{i}^{0}, H_{e, 0}\right) \\
\partial_{t} H_{e}^{m}-\Delta H_{e}^{m} & =-S_{2} H_{e}^{m}+R_{2}^{m}, \quad \text { a.e. } x \in \Omega, m \geq 1 \\
H_{e}^{m}(0, x, \varsigma) & =H_{e, 0}(x, \varsigma), \quad \text { for each } x \in \Omega, \varsigma \in \Sigma_{\epsilon} .
\end{aligned}
$$

Now we show that for almost all $\varsigma \in \Sigma_{\epsilon}$ and for each (not just almost all) fixed $x \in \Omega$, the function $H_{i}^{m}(t)$ is the solution to the corresponding $m$-th equation 18 .

Lemma 3.3. For a.e. $\varsigma \in \Sigma_{\epsilon}$ and for each $x \in \Omega$, if there exists $T<\infty$ such that $H_{e}^{m-1}(\varsigma) \in \mathfrak{H}^{1,1}$ uniquely solves (19) and if $H_{i, 0}(\varsigma) \in L^{2}(\Omega)$, then there exists $H_{i}^{m} \in \mathbb{X}(m \geq 1)$ such that for each $\varsigma \in \Sigma_{\epsilon}$ the function $H_{i}^{m}(\varsigma) \in \mathfrak{H}^{1,0}$ solves uniquely the corresponding $m$-th equation (18). 
Proof. Let $m \geq 1$. Since $F_{2}\left(\chi_{t}\right)$ is $\mathbb{P}_{\varepsilon}$-a.s. uniformly bounded and we assume the existence of the function $H_{e}^{m-1}$, i.e., the unique solution to equation $(19)$, we get that for fixed $\varsigma \in \Sigma_{\varepsilon}, x \in \Omega$, equations (18) define linear inhomogeneous initial value problems. Thus, the unique solution to the $m$-th equation is given by

$$
H_{i}^{m}(t)=e^{\int_{0}^{t} F_{2}^{\varepsilon}\left(\chi_{s}\right) d s}\left(H_{i, 0}+\int_{0}^{t} e^{-\int_{0}^{s} F_{2}^{\varepsilon}\left(\chi_{r}\right) d r} R_{1}^{m-1}(s) d s\right)
$$

More precisely, for each fixed $x \in \Omega$ and $\varsigma \in \Sigma_{\varepsilon}$, we get

$$
H_{i}^{m}(t, x, \varsigma)=e^{\int_{0}^{t} F_{2}^{\varepsilon}\left(\chi_{s}\right) d s}\left(H_{i, 0}(x)+\int_{0}^{t} e^{-\int_{0}^{s} F_{2}^{\varepsilon}\left(\chi_{r}\right) d r} R_{1}^{m-1}(s, x, \varsigma) d s\right) .
$$

Moreover, because of uniform boundedness of the reaction term $R_{1}$ we get

$$
\begin{aligned}
\left|H_{i}^{m}(t, x, \varsigma)\right| & \leq e^{\int_{0}^{t} F_{2}^{\varepsilon}\left(\chi_{s}\right) d s}\left|H_{i, 0}+\int_{0}^{t} e^{-\int_{0}^{s} F_{2}^{\varepsilon}\left(\chi_{r}\right) d r} R_{1}^{m-1}(s, x, \varsigma) d s\right| \\
& \leq e^{C_{F}^{\varepsilon} t}\left|H_{i, 0}(x)\right|+e^{C_{F}^{\varepsilon} t}\left|C_{R_{1}} t\right|, \quad \forall x \in \Omega, \varsigma \in \Sigma_{\epsilon}
\end{aligned}
$$

with $C_{R_{1}}$ denoting the bound for $R_{1}$.

Similarly, for each $x \in \Omega$ and almost all $\varsigma \in \Sigma_{\varepsilon}$, we get that

$$
\left|\partial_{t} H_{i}^{m}(t, x, \varsigma)\right|=\left|R_{1}^{m-1}+H_{i}^{m}(t, x, \varsigma) F_{2}^{\varepsilon}\left(\chi_{t}\right)\right| \leq C_{R_{1}}+H_{i}^{m}(t, \cdot, \varsigma) C_{F}^{\varepsilon}, \quad \forall t \in[0, T],
$$

hence

$$
\left|\partial_{t}\left(H_{i}^{m}\right)\right| \leq\left|C_{R_{1}}+C_{F}^{\varepsilon} H_{i}^{m}\right| .
$$

As $H_{i, 0} \in L^{2}(\Omega)$, we get upon integrating

$$
\left\|H_{i}^{m}(\varsigma)\right\|_{\mathbb{L}_{\Omega, T}}^{2} \leq 2 T e^{2 C_{F}^{\varepsilon} T}\left\|H_{i, 0}\right\|_{L^{2}(\Omega)}^{2}+2 e^{2 C_{F}^{\varepsilon} T} C_{R_{1}}^{2} T^{2}|\Omega|^{2}<\infty
$$

and

$$
\left\|\partial_{t} H_{i}^{m}(\varsigma)\right\|_{\mathbb{L}_{\Omega, T}}^{2} \leq 2 T C_{R_{1}}^{2}|\Omega|^{2}+2\left(C_{F}^{\varepsilon}\right)^{2}\left\|H_{i}^{m}(\varsigma)\right\|_{\mathbb{L}_{\Omega, T}}^{2}<\infty .
$$

From $\sqrt{21}$ and $(22)$ we obtain

$$
\begin{aligned}
\left\|H_{i}^{m}(\varsigma)\right\|_{\mathfrak{H}^{1,0}}^{2} & \leq\left\|H_{i}^{m}(\varsigma)\right\|_{\mathbb{L}_{\Omega, T}}^{2}+\left\|\partial_{t} H_{i}^{m}(\varsigma)\right\|_{\mathbb{L}_{\Omega, T}}^{2} \\
& \leq 2\left(1+2\left(C_{F}^{\varepsilon}\right)^{2}\right) T e^{2 C_{F}^{\varepsilon} T}\left(\left\|H_{i, 0}\right\|_{L^{2}(\Omega)}^{2}+C_{R_{1}}^{2} T|\Omega|^{2}\right)+2 T C_{R_{1}}^{2}|\Omega|^{2}
\end{aligned}
$$

Taking the expectation $\mathbb{E}_{\varepsilon}$ we get

$$
\left\|H_{i}^{m}\right\|_{\mathbb{X}}^{2} \leq 2 T\left(\left(1+\left(C_{F}^{\varepsilon}\right)^{2}\right) e^{2 C_{F}^{\varepsilon} T}\left(\left\|H_{i, 0}\right\|_{\mathbb{L}_{\Sigma, \Omega}}^{2}+C_{R}^{2} T|\Omega|^{2}\right)+C_{R}^{2}|\Omega|^{2}\right),
$$

hence we obtain $H_{i}^{m} \in \mathbb{X}$, such that for almost all $\varsigma \in \Sigma_{\epsilon}$, it is $H_{i}^{m}(\varsigma) \in \mathfrak{H}^{1,0}$. Moreover, for each $x \in \Omega$ fixed, $H_{i}^{m}(t, x, \varsigma)$ uniquely solves the corresponding $m$-th equation (18).

Observe that with a similar argument (under even less restrictive conditions) it can be shown that $H_{i}^{0}(t, x, \varsigma)$ exists as a (unique) solution to the first equation in (18) (with $m=0$ ). Moreover, $H_{e}^{0}(t, x, \varsigma)$ obviously exists as a (unique) solution to the first equation in 19 . These facts allow us to start the following induction procedure:

- Start with $H_{i}^{0}, H_{e}^{0}$.

- For $m \geq 1$ assume $H_{i}^{m} \in \mathfrak{H}^{1,0}$ solves (18), which implies (due to Lemma 3.4 below) the existence of a unique $H_{e}^{m} \in \mathfrak{H}^{1,1}$ solving (19).

- Use $H_{e}^{m}$ found above to prove the existence of $H_{i}^{m+1}$ satisfying for $m \rightsquigarrow m+1$, followed by the existence of $H_{e}^{m+1}$ as solution to 19 ).

Now we can consider the equation 19 in general for $m \in \mathbb{N}$. 
Lemma 3.4. If for each $\varsigma \in \Sigma_{\varepsilon}, H_{e, 0} \in H^{1}(\Omega)$, then there exists some $T>0$ such that $H_{e}^{m}(\varsigma)$ as an element of $\mathfrak{H}^{1,1}$ uniquely solves the corresponding $m$-th equation 119 . Furthermore, $H_{e}^{m} \in \mathbb{Y}$ and it satisfies the following inequality:

$$
\begin{aligned}
\left\|H_{e}^{m}\right\|_{L^{2}\left(0, T ; H^{1}(\Omega)\right)}^{2}+\left\|\partial_{t} H_{e}^{m}\right\|_{\mathbb{L}_{\Omega, T}}^{2} & \leq q_{1}\left\|R_{2}^{m}(\varsigma)\right\|_{\mathbb{L}_{\Omega, T}}^{2}+q_{2}\left\|H_{e, 0}\right\|_{H^{1}(\Omega)}^{2}, \\
\left\|H_{e}^{m}\right\|_{\mathbb{Y}}^{2} & \leq q_{1}\left\|R_{2}^{m}\right\|_{\mathbb{L}_{\Sigma}}^{2}+q_{2}\left\|H_{e, 0}\right\|_{L^{2}\left(\Sigma_{\epsilon} ; H^{1}(\Omega)\right)}^{2},
\end{aligned}
$$

where $q_{1}:=1+\left(1+c_{\epsilon}\right) T e^{S_{2} T}, q_{2}:=q_{1}$ and

$$
L^{2}\left(\Sigma_{\epsilon} ; H^{1}(\Omega)\right):=\left\{u \in L^{2}\left(\Sigma_{\epsilon}\right) \text { such that } u(\varsigma) \in H^{1}(\Omega) \text { a.e. } \varsigma \in \Sigma_{\epsilon}\right\} \text {. }
$$

Proof. The proof is obtained by following the lines of Theorem 7.1.1 in [6]. A detailed proof involving similar arguments will be presented during the existence and uniqueness proof for (CPDM) (in particular for the solution of $9 \mathrm{~b}$ ).

Remark 3. We note here that one can actually even get higher regularity for $H_{e}^{m}$. For $\partial \Omega \in C^{2}$ and $H_{e, 0} \in W^{2, p}(\Omega)$ with $p>d+2$, since $R_{2}^{m} \in L^{\infty}\left(0, T ; L^{\infty}(\Omega)\right.$ ) (see Lemma 3.2) we can apply Theorem 9.1 (Chapter 4 of [25]) and get that for each $\varsigma \in \Sigma_{\epsilon}$,

$$
H_{e}^{m}(\varsigma) \in W(p, T, \Omega):=\left\{u \in L^{p}\left(0, T ; W^{2, p}(\Omega)\right): \partial_{t} u \in L^{p}\left(0, T ; L^{p}(\Omega)\right)\right\} .
$$

Moreover, $H_{e}^{m}$ satisfies the following inequality:

$$
\left\|H_{e}^{m}\right\|_{W(p, T, \Omega)} \leq c(|\Omega|, T, p)\left(\left\|R_{2}^{m}\right\|_{L^{\infty}(\Omega)}+\left\|H_{e, 0}\right\|_{W^{2, p}(\Omega)}\right) .
$$

Thus we get that $\left(H_{e}^{m}\right)_{m \in \mathbb{N}^{*}}$ is a bounded sequence in $W(p, T, \Omega)$. Hence there exists a subsequence $\left(H_{e}^{m_{j}}\right)_{j} \subset\left(H_{e}^{m}\right)_{m \in \mathbb{N}^{*}}$ such that

$$
\begin{gathered}
H_{e}^{m_{j}} \stackrel{j \rightarrow \infty}{\rightarrow} H_{e} \text { in } L^{p}\left(0, T ; W^{2, p}(\Omega)\right) \\
\partial_{t} H_{e}^{m_{j}} \stackrel{j \rightarrow \infty}{\longrightarrow} \partial_{t} H_{e} \text { in } L^{p}\left(0, T ; L^{p}(\Omega)\right) .
\end{gathered}
$$

Moreover, due to the Sobolev embedding $W^{2, p}(\Omega) \hookrightarrow \hookrightarrow L^{p}(\Omega) \hookrightarrow \hookrightarrow L^{2}(\Omega)$ we can apply the Lions-Aubin embedding theorem to get $W(p, T, \Omega) \hookrightarrow \hookrightarrow L^{p}\left(0, T ; L^{p}(\Omega)\right) \hookrightarrow L^{2}\left(0, T ; L^{2}(\Omega)\right)$.

Hence the subsequence $\left(H_{e}^{m_{j}}\right)_{j \in \mathbb{N}} \subset W(p, T, \Omega)$ converges strongly in $L^{p}\left(0, T ; L^{p}(\Omega)\right)$ and $L^{2}\left(0, T ; L^{2}(\Omega)\right)$. For ease of notation, in the $L^{2}\left(0, T ; L^{2}(\Omega)\right)$ convergence proof below we shall ignore the subscript $j$ and just refer the subsequence by $\left(H_{e}^{m}\right)_{m \in \mathbb{N}^{*}}$ itself.

Finally, we get that the limit $H_{e}(\varsigma) \in W(p, T, \Omega) \cap \mathfrak{H}^{1,1}$, for each $\varsigma \in \Sigma_{\epsilon}$. This in turn gives us that

$$
H_{e}(\varsigma) \in C^{1,0}([0, T] \times \bar{\Omega}) \text { and } \nabla H_{e}(\varsigma) \in C([0, T] \times \bar{\Omega}) \text {. }
$$

In particular the uniform continuity of $\nabla H_{e}(\varsigma)$ is used later in the proof of the $H^{2}(\Omega)$ regularity for the solution to (CPDM).

Next we are concerned with the existence of the solution sequences $\left(H_{i}^{m}\right)_{m \in \mathbb{N}} \subset \mathbb{X}$ and $\left(H_{e}^{m}\right)_{m \in \mathbb{N}} \subset \mathbb{Y}$.

Theorem 3.5. Let $C_{F}^{\varepsilon}$ be such that $F_{2}^{\varepsilon}\left(\chi_{t}\right)$ satisfies Lemma 3.1. Then for each $\varsigma \in \Sigma_{\varepsilon}$ and $m \in \mathbb{N}^{*}$ we have that $H_{i}^{m}(\varsigma) \in \mathfrak{H}^{1,0}$ solves uniquely the $m$-th equation specified by (18) and $H_{e}^{m}(\varsigma) \in \mathfrak{H}^{1,1}$ solves uniquely the $m$-th equation specified by 19 . Furthermore, the sequences $\left(H_{i}^{m}\right)_{m \in \mathbb{N}} \subset \mathbb{X}$ and $\left(H_{e}^{m}\right)_{m \in \mathbb{N}} \subset \mathbb{Y}$.

Proof. Follows from lemmas (3.3), (3.4), and the induction procedure. 


\subsubsection{Existence and uniqueness of the solution to (SPDM)}

Consider $\left(H_{e}^{m}\right)_{m \in \mathbb{N}} \subset \mathbb{Y}$, such that for fixed $\varsigma \in \Sigma_{\epsilon}$,

$$
H_{e}^{m}(\varsigma):=\left(H_{e}^{m}(\varsigma)\right)_{m \in \mathbb{N}} \subset \mathfrak{H}^{1,1}
$$

denotes the sequence of solutions to the corresponding $m$-th equation specified by $(19)$.

Similarly, consider $\left(H_{i}^{m}\right)_{m \in \mathbb{N}} \subset \mathbb{X}$, such that for fixed $\varsigma \in \Sigma_{\epsilon}$,

$$
H_{i}^{m}(\varsigma):=\left(H_{i}^{m}(\varsigma)\right)_{m \in \mathbb{N}} \subset \mathfrak{H}^{1,0}
$$

denotes the sequence of solutions to the corresponding $m$-th equation specified by (18).

Next we collect some estimates for the terms in the sequence $H_{e}^{m}$.

Lemma 3.6. For each fixed $\varsigma \in \Sigma_{\varepsilon}$, let $H_{e}^{m}, H_{e}^{n}$ be any two elements of the sequence $\left(H_{e}^{m}\right)_{m}$ (see (24). Then the following inequality holds:

$$
\begin{array}{r}
\left\|H_{e}^{n}(\varsigma)-H_{e}^{m}(\varsigma)\right\|_{L^{2}\left(0, T ; H^{1}(\Omega)\right)}^{2}+\left\|\partial_{t} H_{e}^{n}(\varsigma)-\partial_{t} H_{e}^{m}(\varsigma)\right\|_{\mathbb{L}_{\Omega, T}}^{2} \\
\leq Q_{H_{e}}^{1}\left\|H_{i}^{n}(\varsigma)-H_{i}^{m}(\varsigma)\right\|_{\mathbb{L}_{\Omega, T}}^{2}+Q_{H_{e}}^{2}\left\|H_{e}^{n-1}(\varsigma)-H_{e}^{m-1}(\varsigma)\right\|_{\mathbb{L}_{\Omega, T}}^{2} .
\end{array}
$$

By taking the $\mathbb{E}_{\varepsilon}$ expectation this leads to

$$
\left\|H_{e}^{n}-H_{e}^{m}\right\|_{\mathbb{Y}}^{2} \leq Q_{H_{e}}^{1}\left\|H_{i}^{n}-H_{i}^{m}\right\|_{\mathbb{L}_{\Sigma}}^{2}+Q_{H_{e}}^{2}\left\|H_{e}^{n-1}-H_{e}^{m-1}\right\|_{\mathbb{L}_{\Sigma}}^{2}
$$

where

$$
Q_{H_{e}}^{1}:=q_{1}(T) C_{H_{i}}^{2}, \quad Q_{H_{e}}^{2}:=q_{1}(T) C_{H_{e}}^{2}, \text { and } \quad q_{1}(T):=1+\left(1+\frac{2}{\epsilon}\right) T e^{S_{2} T}
$$

are constants for all fixed $0<T<\infty$.

Proof. Equation 19 applied to the difference $W_{e}^{m, n}:=H_{e}^{m}-H_{e}^{n}$ results in

$$
\partial_{t} W_{e}^{m, n}-\Delta W_{e}^{m, n}+S_{2} W_{e}^{m, n}=R_{2}^{m}-R_{2}^{n} .
$$

Thus the inequality of Lemma 3.4 can be applied to $W_{e}^{m, n}$. Consequently, with $W_{e, 0}^{m, n}=0$ we get

$$
\begin{aligned}
\left\|H_{e}^{m}(\varsigma)-H_{e}^{n}(\varsigma)\right\|_{L^{2}\left(0, T ; H^{1}(\Omega)\right)}^{2} & +\left\|\partial_{t} H_{e}^{n}(\varsigma)-\partial_{t} H_{e}^{m}(\varsigma)\right\|_{\mathbb{L}_{\Omega, T}}^{2} \\
& \leq q_{1}\left\|R_{2}^{m}-R_{2}^{n}\right\|_{\mathbb{L}_{\Omega, T}}^{2} .
\end{aligned}
$$

Applying the Lipschitz continuity of the reaction term $R_{2}$ (see Remark 2 ) we get

$$
\begin{array}{r}
\left\|H_{e}^{m}(\varsigma)-H_{e}^{n}(\varsigma)\right\|_{L^{2}\left(0, T ; H^{1}(\Omega)\right)}^{2}+\left\|\partial_{t} H_{e}^{n}(\varsigma)-\partial_{t} H_{e}^{m}(\varsigma)\right\|_{\mathbb{L}_{\Omega, T}}^{2} \\
\leq q_{1}\left(C_{H_{i}}^{2}\left\|H_{i}^{n}(\varsigma)-H_{i}^{m}(\varsigma)\right\|_{\mathbb{L}_{\Omega, T}}^{2}+C_{H_{e}}^{2}\left\|H_{e}^{n-1}(\varsigma)-H_{e}^{m-1}(\varsigma)\right\|_{\mathbb{L}_{\Omega, T}}^{2}\right) \\
=Q_{H_{e}}^{1}\left\|H_{i}^{n}(\varsigma)-H_{i}^{m}(\varsigma)\right\|_{\mathbb{L}_{\Omega, T}}^{2}+Q_{H_{e}}^{2}\left\|H_{e}^{n-1}(\varsigma)-H_{e}^{m-1}(\varsigma)\right\|_{\mathbb{L}_{\Omega, T}}^{2}
\end{array}
$$

By taking the $\mathbb{E}_{\varepsilon}$ expectation it follows that

$$
\left\|H_{e}^{n}-H_{e}^{m}\right\|_{\mathbb{Y}}^{2} \leq Q_{H_{e}}^{1}\left\|H_{i}^{n}-H_{i}^{m}\right\|_{\mathbb{L}_{\Omega, T}}^{2}+Q_{H_{e}}^{2}\left\|H_{e}^{n-1}-H_{e}^{m-1}\right\|_{\mathbb{L}_{\Omega, T}}^{2} .
$$

Lemma 3.7. Let $H_{e}^{n}, H_{e}^{m}$ be any two arbitrary terms of the sequence $\left(H_{e}^{m}\right)_{m}$. Then it holds that

$$
\begin{aligned}
\left\|H_{e}^{n}(\varsigma)-H_{e}^{m}(\varsigma)\right\|_{\mathbb{L}_{\Omega, T}}^{2} & \leq Q_{H_{e}}^{3}\left\|H_{i}^{n}(\varsigma)-H_{i}^{m}(\varsigma)\right\|_{\mathbb{L}_{\Omega, T}}^{2}+Q_{H_{e}}^{4}\left\|H_{e}^{n-1}(\varsigma)-H_{e}^{m-1}(\varsigma)\right\|_{\mathbb{L}_{\Omega, T}}^{2}, \\
\left\|H_{e}^{n}-H_{e}^{m}\right\|_{\mathbb{L}_{\Sigma}}^{2} & \leq Q_{H_{e}}^{3}\left\|H_{i}^{n}-H_{i}^{m}\right\|_{\mathbb{L}_{\Sigma}}^{2}+Q_{H_{e}}^{4}\left\|H_{e}^{n-1}-H_{e}^{m-1}\right\|_{\mathbb{L}_{\Sigma}}^{2},
\end{aligned}
$$

where

$$
Q_{H_{e}}^{3}:=T e^{T} C_{H_{i}}^{2} \text { and } \quad Q_{H_{e}}^{4}:=T e^{T} C_{H_{e}}^{2}
$$

are constants for all fixed $0<T<\infty$. 
Proof. From (19) we have that for a.e. $\varsigma \in \Sigma_{\varepsilon}$

$$
\begin{aligned}
\int_{\Omega} \partial_{t}\left(H_{e}^{n}-H_{e}^{m}\right) \phi d x & +\int_{\Omega} \nabla\left(H_{e}^{n}-H_{e}^{m}\right) \phi d x+\int_{\Omega} S_{2}\left(H_{e}^{n}-H_{e}^{m}\right) \phi d x \\
& =\int_{\Omega}\left(R_{2}^{n}-R_{2}^{m}\right) \phi d x \quad \forall \phi \in H^{1}(\Omega) .
\end{aligned}
$$

In particular, for $\phi=H_{e}^{n}-H_{e}^{m}$ we get

$$
\begin{aligned}
\frac{1}{2} \frac{d}{d t}\left\|H_{e}^{n}-H_{e}^{m}\right\|_{L^{2}(\Omega)}^{2} & +\left\|\nabla\left(H_{e}^{n}-H_{e}^{m}\right)\right\|_{L^{2}(\Omega)}^{2}+S_{2}\left\|H_{e}^{n}-H_{e}^{m}\right\|_{L^{2}(\Omega)}^{2} \\
& =\int_{\Omega}\left(R_{2}^{n}-R_{2}^{m}\right)\left(H_{e}^{n}-H_{e}^{m}\right) d x,
\end{aligned}
$$

from which

$$
\frac{d}{d t}\left\|H_{e}^{n}-H_{e}^{m}\right\|_{L^{2}(\Omega)}^{2} \leq\left\|R_{2}^{n}-R_{2}^{m}\right\|_{L^{2}(\Omega)}^{2}+\left\|H_{e}^{n}-H_{e}^{m}\right\|_{L^{2}(\Omega)}^{2}
$$

and hence by integrating with respect to $t$ and using the properties of $R_{2}$

$$
\sup _{t \in[0, T]}\left\|H_{e}^{n}-H_{e}^{m}\right\|_{L^{2}(\Omega)}^{2} \leq e^{T}\left(C_{H_{i}}^{2}\left\|H_{i}^{n}-H_{i}^{m}\right\|_{\mathbb{L}_{\Omega, T}}^{2}+C_{H_{e}}^{2}\left\|H_{e}^{n}-H_{e}^{m}\right\|_{\mathbb{L}_{\Omega, T}}^{2}\right),
$$

Thus, by integrating with respect to $t$ and taking the $\mathbb{E}_{\varepsilon}$ expectation

$$
\begin{aligned}
\left\|H_{e}^{n}-H_{e}^{m}\right\|_{\mathbb{L}_{\Omega, T}}^{2} & \leq T e^{T}\left(C_{H_{i}}^{2}\left\|H_{i}^{n}-H_{i}^{m}\right\|_{\mathbb{L}_{\Omega, T}}^{2}+C_{H_{e}}^{2}\left\|H_{e}^{n-1}-H_{e}^{m-1}\right\|_{\mathbb{L}_{\Omega, T}}^{2}\right) \\
\left\|H_{e}^{n}-H_{e}^{m}\right\|_{\mathbb{L}_{\Sigma}}^{2} & \leq T e^{T}\left(C_{H_{i}}^{2}\left\|H_{i}^{n}-H_{i}^{m}\right\|_{\mathbb{L}_{\Sigma}}^{2}+C_{H_{e}}^{2}\left\|H_{e}^{n-1}-H_{e}^{m-1}\right\|_{\mathbb{L}_{\Sigma}}^{2}\right),
\end{aligned}
$$

which proves the claim.

Lemma 3.8. Let $H_{i}^{n}, H_{i}^{m}$ be any two elements of the sequence $\left(H_{i}^{m}\right)_{m}$. Then the difference $H_{i}^{n}-H_{i}^{m}$ satisfies the following inequalities:

$$
\begin{aligned}
\left\|H_{i}^{n}(\varsigma)-H_{i}^{m}(\varsigma)\right\|_{\mathbb{L}_{\Omega, T}}^{2} & \leq Q_{H_{i}}^{3}\left\|H_{i}^{n-1}(\varsigma)-H_{i}^{m-1}(\varsigma)\right\|_{\mathbb{L}_{\Omega, T}}^{2}+Q_{H_{i}}^{4}\left\|H_{e}^{n-1}(\varsigma)-H_{e}^{m-1}(\varsigma)\right\|_{\mathbb{L}_{\Omega, T}}^{2} \\
\left\|H_{i}^{n}-H_{i}^{m}\right\|_{\mathbb{L}_{\Sigma}}^{2} & \leq Q_{H_{i}}^{3}\left\|H_{i}^{n-1}-H_{i}^{m-1}\right\|_{\mathbb{L}_{\Sigma}}^{2}+Q_{H_{i}}^{4}\left\|H_{e}^{n-1}-H_{e}^{m-1}\right\|_{\mathbb{L}_{\Sigma}}^{2}
\end{aligned}
$$

and

$$
\begin{aligned}
\left\|H_{i}^{n}(\varsigma)-H_{i}^{m}(\varsigma)\right\|_{\mathfrak{H}^{1,0},}^{2} & \leq Q_{H_{i}}^{1}\left\|H_{i}^{n-1}(\varsigma)-H_{i}^{m-1}(\varsigma)\right\|_{\mathbb{L}_{\Omega, T}}^{2}+Q_{H_{i}}^{2}\left\|H_{e}^{n-1}(\varsigma)-H_{e}^{m-1}(\varsigma)\right\|_{\mathbb{L}_{\Omega, T}}^{2}, \\
\left\|H_{i}^{n}-H_{i}^{m}\right\|_{\mathbb{X}}^{2} & \leq Q_{H_{i}}^{1}\left\|H_{i}^{n-1}-H_{i}^{m-1}\right\|_{\mathbb{L}_{\Sigma}^{\infty}}^{2}+Q_{H_{i}}^{2}\left\|H_{e}^{n-1}-H_{e}^{m-1}\right\|_{\mathbb{L}_{\Sigma}}^{2},
\end{aligned}
$$

where

$$
\begin{array}{cc}
r_{1}:=e^{4 T^{2}\left(C_{F}^{\varepsilon}\right)^{2},} & Q_{H_{i}}^{3}:=4 \operatorname{Tr}_{1} C_{H_{i}}^{2}, \quad Q_{H_{i}}^{4}:=4 T r_{1} C_{H_{e}}^{2}, \\
Q_{H_{i}}^{1}:=4\left(1+\left(C_{F}^{\varepsilon}\right)^{2}\right) Q_{H_{i}}^{3}+4 C_{H_{i}}^{2}, & Q_{H_{i}}^{2}:=4\left(1+\left(C_{F}^{\varepsilon}\right)^{2}\right) Q_{H_{i}}^{4}+4 C_{H_{e}}^{2} .
\end{array}
$$

are constants and $T>0$ is the right end of a fixed time interval $[0, T]$.

Proof. Since the elements of the sequence $\left(H_{i}^{m}(\varsigma)\right)_{m}$ are solutions to the respective $m$-th equation specified by (18), for each fixed $x \in \Omega$ and a.e. $\varsigma \in \Sigma_{\varepsilon}$ we have that

$$
\left|H_{i}^{n}(t, x, \varsigma)-H_{i}^{m}(t, x, \varsigma)\right|=\left|\int_{0}^{t}\left(R_{1}^{n-1}-R_{1}^{m-1}\right) d t+\int_{0}^{t} F_{2}^{\varepsilon}\left(\chi_{t}\right)\left(H_{i}^{n}-H_{i}^{m}\right) d t\right|,
$$

from which, by using the Lipschitz continuity of the reaction term $R_{1}$ (see Remark 2), we get

$$
\left|H_{i}^{n}-H_{i}^{m}\right| \leq \int_{0}^{t}\left(C_{H_{i}}\left|H_{i}^{n-1}-H_{i}^{m-1}\right|+C_{H_{e}}\left|H_{e}^{n-1}-H_{e}^{m-1}\right|+C_{F}^{\varepsilon}\left|H_{i}^{n}-H_{i}^{m}\right|\right) d t,
$$

from which

$$
\left|H_{i}^{n}-H_{i}^{m}\right|^{2} \leq a(T)+4 T\left(C_{F}^{\varepsilon}\right)^{2} \int_{0}^{t}\left|H_{i}^{n}-H_{i}^{m}\right|^{2} d t
$$


where

$$
a(T):=4 \int_{0}^{T} C_{H_{i}}^{2}\left|H_{i}^{n-1}-H_{i}^{m-1}\right|^{2} d t+4 \int_{0}^{T} C_{H_{e}}^{2}\left|H_{e}^{n-1}-H_{e}^{m-1}\right|^{2} d t .
$$

Applying Gronwall's inequality and integrating with respect to $x$ it follows

$$
\left\|H_{i}^{n}-H_{i}^{m}\right\|_{L^{2}(\Omega)}^{2} \leq\left(e^{4 T\left(C_{F}^{\varepsilon}\right)^{2} T}\right) \int_{\Omega} a(T) d x .
$$

This implies

$$
\left\|H_{i}^{n}-H_{i}^{m}\right\|_{L^{2}(\Omega)}^{2} \leq 4 r_{1} C_{H_{i}}^{2}\left\|H_{i}^{n-1}-H_{i}^{m-1}\right\|_{\mathbb{L}_{\Omega, T}}^{2}+4 r_{1} C_{H_{e}}^{2}\left\|H_{e}^{n-1}-H_{e}^{m-1}\right\|_{\mathbb{L}_{\Omega, T}}^{2},
$$

hence

$$
\begin{aligned}
\left\|H_{i}^{n}-H_{i}^{m}\right\|_{\mathbb{L}_{\Omega, T}}^{2} & \leq 4 r_{1} T\left(C_{H_{i}}^{2}\left\|H_{i}^{n-1}-H_{i}^{m-1}\right\|_{\mathbb{L}_{\Omega, T}}^{2}+C_{H_{e}}^{2}\left\|H_{e}^{n-1}-H_{e}^{m-1}\right\|_{L^{2}\left(0, T ; L^{2}(\Omega)\right)}^{2}\right) \\
& =Q_{H_{i}}^{3}\left\|H_{i}^{n-1}-H_{i}^{m-1}\right\|_{\mathbb{L}_{\Omega, T}}^{2}+Q_{H_{i}}^{4}\left\|H_{e}^{n-1}-H_{e}^{m-1}\right\|_{\mathbb{L}_{\Omega, T}}^{2}
\end{aligned}
$$

with $r_{1}:=\exp \left(4 T^{2}\left(C_{F}^{\varepsilon}\right)^{2}\right), Q_{H_{i}}^{3}:=4 T r_{1} C_{H_{i}}^{2}, Q_{H_{i}}^{4}:=4 T r_{1} C_{H_{e}}^{2}$.

For each fixed $x \in \Omega$ and $\varsigma \in \Sigma_{\epsilon}$ the derivative can be estimated in a similar way to yield:

$$
\begin{gathered}
\left\|\partial_{t}\left(H_{i}^{n}-H_{i}^{m}\right)\right\|_{\mathbb{L}_{\Omega, T}}^{2} \leq 4 C_{H_{i}}^{2}\left\|H_{i}^{n-1}-H_{i}^{m-1}\right\|_{\mathbb{L}_{\Omega, T}}^{2}+4 C_{H_{e}}^{2}\left\|H_{e}^{n-1}-H_{e}^{m-1}\right\|_{\mathbb{L}_{\Omega, T}}^{2} \\
+4\left(C_{F}^{\varepsilon}\right)^{2}\left\|H_{i}^{n}-H_{i}^{m}\right\|_{\mathbb{L}_{\Omega, T}}^{2}
\end{gathered}
$$

Substituting (30) in the above equation results in

$$
\leq\left(4\left(C_{F}^{\varepsilon}\right)^{2} Q_{H_{i}}^{3}+4 C_{H_{i}}^{2}\right)\left\|H_{i}^{n-1}-H_{i}^{m-1}\right\|_{\mathbb{L}_{\Omega, T}}^{2}+\left(4\left(C_{F}^{\varepsilon}\right)^{2} Q_{H_{i}}^{4}+4 C_{H_{e}}^{2}\right)\left\|H_{e}^{n-1}-H_{e}^{m-1}\right\|_{\mathbb{L}_{\Omega, T}}^{2}
$$

Altogether, we have that

$$
\begin{aligned}
\left\|H_{i}^{n}-H_{i}^{m}\right\|_{\mathfrak{H}^{1,0}}^{2} & \leq\left\|H_{i}^{n}-H_{i}^{m}\right\|_{\mathbb{L}_{\Omega, T}}^{2}+\left\|\partial_{t} H_{i}^{n}-H_{i}^{m}\right\|_{\mathbb{L}_{\Omega, T}}^{2} \\
& \leq Q_{H_{i}}^{1}\left\|H_{i}^{n-1}-H_{i}^{m-1}\right\|_{\mathbb{L}_{\Omega, T}}^{2}+Q_{H_{i}}^{2}\left\|H_{e}^{n-1}-H_{e}^{m-1}\right\|_{\mathbb{L}_{\Omega, T}}^{2}
\end{aligned}
$$

with

$$
Q_{H_{i}}^{1}:=4\left(1+\left(C_{F}^{\varepsilon}\right)^{2}\right) Q_{H_{i}}^{3}+4 C_{H_{i}}^{2} \text { and } Q_{H_{i}}^{2}:=4\left(1+\left(C_{F}^{\varepsilon}\right)^{2}\right) Q_{H_{i}}^{4}+4 C_{H_{e}}^{2} .
$$

The last two inequalities prove the claim.

The immediate consequence of Lemmas 3.7 and 3.8 is the following sufficient condition for the convergence of the sequence $\left(H_{i}^{m}, H_{e}^{m}\right)$ in $\mathbb{X} \times \mathbb{Y}$.

Lemma 3.9 (SPDM time condition). For the sequence $\left(H_{i}^{m}(\varsigma), H_{e}^{m}(\varsigma)\right)_{m}$ to be a Cauchy sequence in $\mathfrak{H}^{1,0} \times \mathfrak{H}^{1,1}$, the time $T$ has to fulfill the following condition:

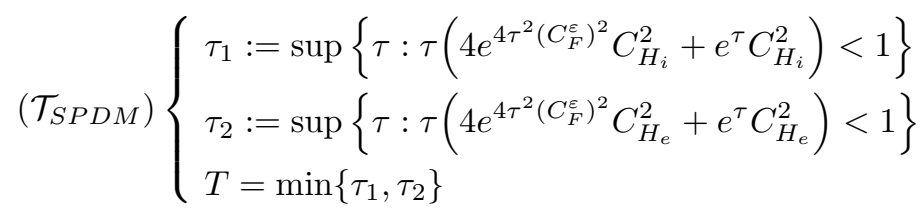

Proof. Since for a fixed $T>0$ the constants $Q_{H_{i}}^{1}, Q_{H_{i}}^{2}, Q_{H_{e}}^{1}$ and $Q_{H_{e}}^{2}$ occurring in Lemmas 3.7 and 3.8 respectively, are all bounded, we get that the sequence $\left(H_{i}^{m}, H_{e}^{m}\right)$ converges in $\mathbb{X} \times \mathbb{Y}$ if and only if $\left(H_{i}^{m}, H_{e}^{m}\right)$ converges in $\mathbb{L}_{\Sigma} \times \mathbb{L}_{\Sigma}$. Hence it is sufficient to find a condition for the latter.

$$
\begin{aligned}
\left\|H_{i}^{n}-H_{i}^{m}\right\|_{\mathbb{L}_{\Sigma}}^{2}+\left\|H_{e}^{n}-H_{e}^{m}\right\|_{\mathbb{L}_{\Sigma}} & \leq\left(Q_{H_{i}}^{3}+Q_{H_{e}}^{3}\right)\left\|H_{i}^{n-1}(\varsigma)-H_{i}^{m-1}(\varsigma)\right\|_{\mathbb{L}_{\Sigma}}^{2} \\
& +\left(Q_{H_{i}}^{4}+Q_{H_{e}}^{4}\right)\left\|H_{e}^{n-1}(\varsigma)-H_{e}^{m-1}(\varsigma)\right\|_{\mathbb{L}_{\Sigma}}^{2} .
\end{aligned}
$$

with $Q_{H_{i}}^{3}:=4 T e^{4 T^{2}\left(C_{F}^{\varepsilon}\right)^{2}} C_{H_{i}}^{2}, Q_{H_{e}}^{3}:=T e^{T} C_{H_{i}}^{2}, Q_{H_{i}}^{4}:=4 T e^{4 T^{2}\left(C_{F}^{\varepsilon}\right)^{2}} C_{H_{e}}^{2}$, and $Q_{H_{e}}^{4}:=T e^{T} C_{H_{e}}^{2}$. Consequently, we have the condition $\left(\mathcal{T}_{S P D M}\right)$ for the time $T$. 
Finally, we are in a position to formulate the existence theorem.

Theorem 3.10. For each parameter vector $\Xi$, there exists a time interval $[0, T)$ with $T>0$ satisfying the time condition $\left(\mathcal{T}_{S P D M}\right)$ (see (31)), such that (SPDM) has a unique weak solution $H_{i}(t, x, \varsigma), H_{e}(t, x, \varsigma)$ for almost every $\varsigma \in \Sigma_{\epsilon}$. Furthermore $H_{i}(\cdot, \cdot, \varsigma)$ and $H_{e}(\cdot, \cdot, \varsigma)$ are the sample paths of the processes $\left(H_{i}(t)\right)_{t \in[0, T]} \in \mathbb{X}$ and $\left(H_{e}(t)\right)_{t \in[0, T]} \in \mathbb{Y}$, and these processes are a.s. unique in $\mathbb{X}$ and $\mathbb{Y}$, respectively.

In addition, if $\Omega \subset \mathbb{R}^{d}$ with $\partial \Omega$ being $C^{2}$ and $H_{e, 0}(\varsigma) \in W^{2, p}(\Omega)$ with $p>d+2$, then (due to Remark [3) $H_{e} \in W(p, T, \Omega)$.

Proof. From Lemma 3.9 we have that $\left(H_{i}^{m}(\varsigma), H_{e}^{m}(\varsigma)\right)_{m}$ is a Cauchy sequence in $\mathfrak{H}^{1,0} \times \mathfrak{H}^{1,1}$, thus it converges to a unique element $\left(H_{i}(\varsigma), H_{e}(\varsigma)\right)$ in $\mathfrak{H}^{1,0} \times \mathfrak{H}^{1,1}$ for a.e. $\varsigma \in \Sigma_{\epsilon}$. Then by dominated convergence we obtain that $\left(H_{i}^{m}, H_{e}^{m}\right)$ converges to $\left(H_{i}, H_{e}\right)$ in $\mathbb{X} \times \mathbb{Y}$.

For uniqueness let $H_{i, 1}, H_{i, 2} \in \mathbb{X}$ be such that almost every corresponding sample path is a weak solution to 8a). Then for a fixed $\varsigma \in \Sigma_{\epsilon}$ we obtain $\left\|H_{i, 1}-H_{i, 2}\right\|_{\mathbb{L}_{\Sigma}}^{2}=0$ by employing the usual estimates and taking the $\mathbb{E}_{\varepsilon}$ expectation and due to the Lipschitz continuity of $R_{1}$. Therefore, $H_{i, 1}, H_{i, 2} \in \mathbb{X}$ are a.s. identical in $\mathbb{L}_{\Sigma}$. Now from the estimate 290 in Lemma 3.8 , the uniqueness of $H_{e}$ in $\mathbb{L}_{\Sigma}$ (shown below) and since $\mathbb{X} \subset \mathbb{L}_{\Sigma}$, we can conclude that $H_{i, 1}$ and $H_{i, 2}$ are also a.s. identical in $\mathbb{X}$.

For the uniqueness of $H_{e} \in \mathbb{Y}$, let $H_{e, 1}, H_{e, 2} \in \mathbb{X}$ be such that almost every sample path is a weak solution to (8b). Then for every fixed $\varsigma \in \Sigma_{\epsilon}$ and due to the Lipschitz continuity of $R_{2}$ we obtain in the usual way (after also taking the $\mathbb{E}_{\varepsilon}$ expectation) that $\left\|H_{e, 1}-H_{e, 2}\right\|_{\mathbb{L}_{\Sigma}}^{2}=0$. Hence $H_{e, 1}, H_{e, 2} \in \mathbb{Y}$ are a.s. identical in $\mathbb{L}_{\Sigma}$. Moreover, due to (26), the uniqueness of $H_{i}$ in $\mathbb{L}_{\Sigma}$ and the fact that $\mathbb{Y} \subset \mathbb{L}_{\Sigma}$ we can conclude that $H_{e, 1}$ and $H_{e, 2}$ are also a.s. identical in $\mathbb{Y}$.

\subsubsection{Measurability of the solution to (SPDM)}

Now we show that the processes $H_{i} \in \mathbb{X}$ and $H_{e} \in \mathbb{Y}$ are adapted to the filtration $\mathcal{A}_{t}^{\varepsilon}$ generated by the noise process $\left(F_{2}^{\varepsilon}\left(\chi_{t}\right)\right)_{t \in[0, T]}$, with $T$ satisfying the condition 31.

Lemma 3.11. Let $t \in[0, T]$ with $T>0$ satisfying (31). If $H_{i}^{m-1} \in\left(H_{i}^{m}\right)_{m}$ is an a.s. $\mathcal{A}_{t}^{\varepsilon}$ adapted process and $H_{e}^{m-1} \in\left(H_{e}\right)_{m}$ is an $\mathcal{A}_{t}^{\varepsilon}$ adapted process, then $H_{i}^{m} \in\left(H_{i}^{m}\right)_{m}$ is an a.s. $\mathcal{A}_{t}^{\varepsilon}$ adapted process.

Proof. Let $H_{i}^{m}$ be an arbitrary element of the sequence $\left(H_{i}^{m}\right)_{m}$. Due to Lemma 3.3 , for each $x \in \Omega$ and almost all $\varsigma \in \Sigma_{\epsilon}$

$$
\frac{d}{d t} H_{i}^{m}(t)=R_{1}\left(H_{i}^{m-1}, H_{e}^{m-1}\right)+H_{i}^{m}(t) F_{2}^{\varepsilon}\left(\chi_{t}\right),
$$

hence

$$
H_{i}^{m}(t)=\exp \left(\int_{0}^{t} F_{2}^{\varepsilon}\left(\chi_{s}\right) d s\right)\left(H_{i, 0}+\int_{0}^{t} e^{-\int_{s}^{t} F_{2}^{\varepsilon}\left(\chi_{r}\right) d r} R_{1}^{m-1}(s) d s\right) .
$$

Thus for every fixed $x \in \Omega$ and for almost all $\varsigma \in \Sigma_{\epsilon}, H_{i}^{m}(t)$ depends on $H_{i}^{m-1}(s), H_{e}^{m-1}(s)$, and $F_{2}^{\varepsilon}\left(\chi_{s}\right)$ for $s \in[0, t]$. So if $H_{i}^{m-1}(t)$ and $H_{e}^{m-1}(t)$ are $\mathcal{A}_{t}^{\varepsilon}$ measurable then so is $H_{i}^{m}(t)$. Since this holds for each $x \in \Omega$ and almost all $\varsigma \in \Sigma_{\epsilon}$, we find that $H_{i}^{m} \in \mathbb{X}$ is a.s. an $\mathcal{A}_{t}^{\varepsilon}$ adapted process.

In particular, we readily see that $H_{i}^{0}$ is an a.s. $\mathcal{A}_{t}^{\varepsilon}$ adapted process, due the linear inhomogeneous equation it satisfies. This indicates us to invoke the induction procedure so that the a.s. $\mathcal{A}_{t}^{\varepsilon}$ adaptability of an arbitrary process $H_{e}^{m} \in\left(H_{e}^{m}\right)_{m}$ can be obtained by assuming that $H_{i}^{m}$ and $H_{e}^{m-1}$ are a.s. $\mathcal{A}_{t}^{\varepsilon}$ adapted processes. Next lemma verifies the latter half of the previous statement.

Lemma 3.12. Let $t \in[0, T]$ with $T>0$ satisfying (31). If $H_{i}^{m} \in\left(H_{i}^{m}\right)_{m}$ is an a.s. $\mathcal{A}_{t}^{\varepsilon}$ adapted process and $H_{e}^{m-1} \in\left(H_{e}^{m}\right)_{m}$ is an $\mathcal{A}_{t}^{\varepsilon}$ adapted process, then $H_{e}^{m} \in\left(H_{e}^{m}\right)_{m}$ is an a.s. $\mathcal{A}_{t}^{\varepsilon}$ adapted process. 
Proof. By the hypothesis it follows that $H_{i}^{m}(t), H_{e}^{m-1}(t)$ are $\mathcal{A}_{t}^{\varepsilon}$ measurable, for $t \in[0, T]$ and $T$ satisfying (31). Recalling equation (19) for $m \in \mathbb{N}_{0}$ we have that

$$
\int_{\Omega} H_{e}^{m} \phi d x+\int_{\Omega} \nabla H_{e}^{m} \cdot \nabla \phi d x+S_{2} \int_{\Omega} H_{e}^{m} \phi d x=R_{2}\left(H_{i}^{m}, H_{e}^{m-1}\right), \quad \forall \phi \in H^{1}(\Omega) .
$$

For fixed $\varsigma \in \Sigma_{\epsilon}$, the function $H_{e}^{m}(\varsigma) \in H^{1}\left([0, T] ; H^{1}(\Omega)\right)$ can be written as

$$
H_{e}^{m}(t, x, \varsigma):=\sum_{k=0}^{\infty} d_{k}^{m}(t, \varsigma) w_{k}(x), \quad \sum_{k=0}^{\infty}\left|d_{k}^{m}(t, \varsigma)\right|^{2}<\infty
$$

where $\left(w_{k}\right)_{k \in \mathbb{N}_{0}}$ is a complete orthogonal basis of the Hilbert space $H^{1}(\Omega)$.

Due to the embedding $H^{1} \subset L^{2} \subset\left(H^{1}(\Omega)\right)^{*}$, the function $\left(H_{e}^{m}\right)^{\prime}(t) \in\left(H^{1}(\Omega)\right)^{*} \cap L^{2}(\Omega)$ can also be characterized by using the same (now orthonormal) basis in $L^{2}$ as

$$
\left(H_{e}^{m}\right)^{\prime}(t, x, \varsigma):=\sum_{k=0}^{\infty}\left(d_{k}^{m}\right)^{\prime}(t, \varsigma) w_{k}(x), \quad \sum_{k=0}^{\infty}\left|\left(d_{k}^{m}\right)^{\prime}(t, \varsigma)\right|^{2}<\infty .
$$

By the same argument we can represent $R_{2}\left(X^{m}, Y^{m-1}\right)$ as

$$
R_{2}^{m}(t, x, \varsigma):=\sum_{k=0}^{\infty} f_{k}^{m, m-1}(t, \varsigma) w_{k}(x), \quad \sum_{k=0}^{\infty}\left|f_{k}^{m, m-1}(t, \varsigma)\right|^{2}<\infty .
$$

Using this representation for $H_{e}^{m}(\varsigma)$, we get the following equation :

$$
\begin{array}{r}
\sum_{j=0}^{\infty}\left(d_{j}^{m}\right)^{\prime}(t) \int_{\Omega} w_{j}(x) \phi d x+\sum_{j=0}^{\infty} d_{j}^{m}(t) \int_{\Omega} \nabla w_{j}(x) \cdot \nabla \phi d x \\
+S_{2} \sum_{j=0}^{\infty} d_{j}^{m}(t) \int_{\Omega} w_{j}(x) \phi d x=\sum_{j=0}^{\infty} f_{j}^{m, m-1}(t) \int_{\Omega} w_{j}(x) \phi d x .
\end{array}
$$

In particular for $\phi=w_{k}$ we get

$$
\left(d_{k}^{m}\right)^{\prime}(t)+\sum_{j=0}^{\infty} d_{j}^{m}(t) \int_{\Omega} \nabla w_{j}(x) \cdot \nabla w_{k}(x) d x+S_{2} d_{k}^{m}(t)=f_{k}^{m, m-1}(t) .
$$

Define $a_{j, k}=\int_{\Omega} \nabla w_{j} \cdot \nabla w_{k} d x$; then we get

$$
\left(d_{k}^{m}\right)^{\prime}(t)+\sum_{j=0}^{\infty} d_{j}^{m}(t) a_{j, k}+S_{2} d_{k}^{m}(t)=f_{k}^{m, m-1}(t) .
$$

Since

$$
\left|\left(d_{k}^{m}\right)^{\prime}(t)\right|=\left|\left(\left(H_{e}^{m}\right)^{\prime}(t), w_{k}\right)\right|<\infty \quad \text { and } \quad\left|d_{k}^{m}(t)\right|=\left|\left(H_{e}^{m}(t), w_{k}\right)\right|<\infty
$$

and

$$
\left|\left(R_{2}^{m, m-1}(t), w_{k}\right)\right|<\infty,
$$

we get that

$$
\left|\sum_{j=0}^{\infty} d_{j}^{m}(t) a_{j, k}\right|=\left|f_{k}^{m, m-1}(t)-\left(d_{k}^{m}\right)^{\prime}(t)-S_{2} d_{k}^{m}(t)\right|<\infty .
$$

Thus equation (37) with infinite sum makes sense and we can represent the solution using the exponential of a matrix.

Let $\mathbf{d}^{m}=\left(d_{0}^{m}, \ldots, d_{k}^{m}, \ldots,\right)^{T}, \mathbf{f}^{m, m-1}=\left(f_{0}^{m, m-1}, \ldots, f_{k}^{m, m-1}, \ldots,\right)^{T}, k \in \mathbb{N}$, and $\mathbf{A}=\left(\left(a_{i, j}\right)_{i, j \in \mathbb{N}}\right)$, then $(37)$ can be represented in vector form as:

$$
\left(\mathbf{d}^{m}\right)^{\prime}(t)=-\left(\mathbf{A}+S_{2} \mathbf{I}\right) \mathbf{d}^{m}(t)+\mathbf{f}^{m, m-1}(t),
$$


whose solution is given as

$$
\mathbf{d}^{m}(t)=e^{-\left(\mathbf{A}+S_{2} \mathbf{I}\right) t}\left(\mathbf{d}^{m}(0)+\int_{0}^{t} e^{\left(\mathbf{A}+S_{2} \mathbf{I}\right) s}\left(\mathbf{f}^{m, m-1}(s)\right) d s\right)
$$

This implies that each $d_{k}^{m}(t)$ depends on $f_{k}^{m, m-1}(s), s \in[0, t]$. Consequently, $H_{e}^{m}(t)$ also depends on $f_{k}^{m, m-1}(s), s \in[0, t]$ for almost all $\varsigma \in \Sigma_{\epsilon}$. Because $R_{2}\left(H_{i}^{m}, H_{e}^{m-1}\right)$ is a smooth function of $H_{i}^{m}, H_{e}^{m-1}$ it is also an $\mathcal{A}_{t}^{\varepsilon}$-adapted process. Since the pathwise characterization (34) preserves this property, we can conclude that $H_{e}^{m}(t) \in \mathbb{Y}$ is an a.s. $\mathcal{A}_{t}^{\varepsilon}$ adapted process.

Combining lemmas 3.11 and 3.12 along with the induction argument, we get that the elements of the sequence $\left(H_{i}^{m}\right)_{m},\left(H_{e}^{m}\right)_{m}$ are a.s. $\mathcal{A}_{t}^{\varepsilon}$-adapted processes. Thus, the corresponding limit processes $H_{i} \in \mathbb{X}$ and $H_{e} \in \mathbb{Y}$ are also a.s. $\mathcal{A}_{t}^{\varepsilon}$ adapted.

\subsubsection{Uniform boundedness of the solution to (SPDM)}

In this section we prove the boundedness and nonnegativity of the solutions to (SPDM), which will be used in the proof for the well posedness of the macroscopic model.

Theorem 3.13. Let $\hat{T}>0$ be the maximum time value satisfying the time condition $\mathcal{T}_{S P D M}$ (see (31) below). Then for every parameter vector $\Xi$ there exists a time $\tilde{T}>0$ such that for

$$
T:=\min (\tilde{T}, \hat{T}),
$$

the solution sequences $\left(H_{i}^{m}\right)_{m}$ and $\left(H_{e}^{m}\right)_{m}$ are uniformly bounded with the following upper and lower bounds:

1. If $H_{i}^{m}(0, x, \varsigma) \geq 0 \forall x \in \Omega$ then for each $m \in \mathbb{N}$

$$
\begin{gathered}
0 \leq H_{i}^{m}(t, x, \varsigma) \leq C_{H_{i}}(T) \forall x \in \Omega, t \in[0, T), \varsigma \in \Sigma_{\epsilon} \\
C_{H_{i}}(T):=e^{C_{F}^{\varepsilon} T}\left(\sup _{x \in \Omega} H_{i, 0}(x)+C_{R_{1}} T\right) .
\end{gathered}
$$

2. If $H_{e}^{m}(0, x, \varsigma) \geq 0 \forall x \in \Omega$ then for each $m \in \mathbb{N}$

$$
\begin{gathered}
c_{H_{e}}(T) \leq H_{e}^{m}(t, x, \varsigma) \leq C_{H_{e}}(T) \forall x \in \Omega, t \in[0, T), \varsigma \in \Sigma_{\epsilon} \\
c_{H_{e}}(T):=a e^{-b_{1} T}, \quad C_{H_{e}}(T):=e^{b_{1} T},
\end{gathered}
$$

where the constants $b_{1}$ and $b_{2}$ will be specified in the proof.

Proof. Let $H_{e}^{m} \in\left(H_{e}^{m}\right)_{m}$ and $H_{i}^{m} \in\left(H_{i}^{m}\right)_{m}$. Observe that the assertions are obviously verified for $H_{i}^{0}$ and $H_{e}^{0}$. Consider the equation

$$
\partial_{t} H_{i}^{m}(t)=R_{1}^{m-1}(t)+F\left(\chi_{t}, H_{i}^{m}\right),
$$

with $F\left(\chi_{t}, H_{i}^{m}\right)=H_{i}^{m} F_{2}^{\varepsilon}\left(\chi_{t}\right)$ and whose solution is given as in $(20)$. Assume the assertions of the theorem hold for $H_{i}^{m-1}$ and $H_{e}^{m-1}$. Then they also hold for $H_{i}^{m}$, by the estimates in Lemma 3.3 and the announced choice of $C_{H_{i}}(T)$.

Now consider the equation

$$
\partial_{t} H_{e}^{m}(t, x, \varsigma)=R_{2}^{m}-S_{2} H_{e}^{m}+\Delta H_{e}^{m} .
$$

For each fixed $\varsigma \in \Sigma_{\epsilon}$, let $\tilde{H}_{e}^{m}(t, x):=H_{e}^{m}(t, x)-a e^{-b_{1} t}$. Then

$$
\begin{aligned}
\partial_{t} \tilde{H}_{e}^{m}-\Delta \tilde{H}_{e}^{m}+S_{2} \tilde{H}_{e}^{m} & =R_{2}^{m}+a e^{-b_{1} t}\left(q-S_{2}\right) \\
& \geq q_{R_{2}}+a e^{-b_{1} t}\left(b_{1}-S_{2}\right), \text { since } R_{2}^{m} \geq q_{R_{2}} \text { with } 0>q_{R_{2}} \\
& =a\left(\hat{q} e^{-\left(\hat{b}_{1}+S_{2}\right) t}-1\right), \text { with } a:=\left|q_{R_{2}}\right|, \hat{b}_{1}=b_{1}-S_{2} .
\end{aligned}
$$

For each $\hat{q}>1$ there exists $\tilde{T}>0$ such that

$$
\hat{b}_{1} e^{-\left(\hat{b}_{1}+S_{2}\right) \tilde{T}}>1 \text {. }
$$


Therefore,

$$
\partial_{t} \tilde{H}_{e}^{m}-\Delta \tilde{H}_{e}^{m}+S_{2} \tilde{H}_{e}^{m} \geq 0 \forall t \in[0, \tilde{T}) .
$$

So if $H_{e, 0} \geq a e^{-b_{1} t}$ then $\tilde{H}_{e}^{m}(0, x) \geq 0$, hence e.g., from the nonnegativity theorem in [26] we get that $\tilde{H}_{e}^{m}(t, x) \geq 0$ for all $t \in[0, \tilde{T}), x \in \Omega$. This in turn implies that for

$$
T:=\min (\hat{T}, \tilde{T})
$$

$H_{e}^{m}(t, x) \geq c_{H_{e}}(T)$ for all $t \in[0, T), x \in \Omega$, and $\varsigma \in \Sigma_{\epsilon}$, with $c_{H_{e}}(T):=a e^{-q T}$.

Now for each fixed $\varsigma \in \Sigma_{\epsilon}$, let

$$
\hat{H}_{e}^{m}(t, x):=e^{b_{2} t}-H_{e}^{m}(t, x),
$$

then

$$
\begin{aligned}
\partial_{t} \hat{H}_{e}^{m}-\Delta \hat{H}_{e}^{m}+S_{2} \hat{H}_{e}^{m} & =b_{2} e^{b_{2} t}+S_{2} e^{b_{2} t}-R_{2}^{m} \\
& \geq e^{b_{2} t}\left(S_{2}+b_{2}\right)-Q_{R_{2}},
\end{aligned}
$$

since $R_{2}^{m} \leq Q_{R_{2}}$ with $Q_{R_{2}}>0$. Thus, for $b_{2} \geq Q_{R_{2}}$ it follows that

$$
\partial_{t} \hat{H}_{e}^{m}-\Delta \hat{H}_{e}^{m}+S_{2} \hat{H}_{e}^{m} \geq 0, \forall t \in[0, T), x \in \Omega, \varsigma \in \Sigma_{\epsilon} .
$$

Hence, once again by the nonnegativity theorem in [26] we get $\hat{H}_{e}^{m}(t, x) \geq 0$ for all $t \in[0, T)$ and $x \in \Omega$. This in turn implies that $H_{e}^{m}(t, x) \leq C_{H_{e}}(T):=e^{b_{2} T}$ for all $t \in[0, T)$ and $x \in \Omega$. (Take $b_{2}>\max \left\{1+S_{2}, Q_{R_{2}}\right\}$.) Altogether we obtain that $0 \leq H_{e}^{m}(t, x, \varsigma) \leq C_{H_{e}}(T) \forall t \in[0, T), x \in \Omega$, $\varsigma \in \Sigma_{\epsilon}$.

This completes the proof for the uniform boundedness of the solutions to (SPDM). The next step is to analyze the wellposedness of CPDM. This shall be the topic of next section.

\subsection{Analysis of the cell population dynamics model (CPDM)}

The goal of this section is to prove the local existence of a unique solution to (CPDM), from which we get a new condition for the maximum time interval $[0, T)$ for the existence of a unique solution to the full stochastic multiscale model.

The following lemma is directly obtained by applying Theorem 3.13 .

Lemma 3.14. (Boundedness of $\Lambda_{1}, \Lambda_{2}$ and $D$ ) Let $T>0$ satisfy the condition (38) and let $C, N$ be non-negative and bounded. Then the proliferation (or switching) function $\Lambda_{1}\left(\overline{H_{i}}\right)$, the decay function $\Lambda_{2}\left(H_{e}\right)$ and the diffusion coefficient $D(C, N)$ are uniformly bounded:

$$
\begin{gathered}
0 \leq \Lambda_{1}\left(H_{i}\right) \leq C_{\Lambda_{1}}:=\omega_{1} c_{3} \exp \left(c_{1}\left(1+C_{H_{i}}\right)\right), \\
0 \leq \Lambda_{2}\left(H_{i}\right) \leq C_{\Lambda_{2}}:=\omega_{2} \log _{2.15}\left(1+C_{H_{e}}\right), \\
0<c_{D} \leq D(C, N) \leq \gamma
\end{gathered}
$$

for all $x \in \Omega, t \in[0, T]$, and $\varsigma \in \Sigma_{\epsilon}$.

\subsubsection{Iterative solution sequence for (CPDM)}

In order to find the pathwise weak solution to (CPDM), that is for each $\varsigma \in \Sigma_{\epsilon}$ find $N(\varsigma) \in \mathfrak{H}^{1,0}$ and $C(\varsigma) \in \mathfrak{H}^{1,2}$, we rewrite the equations $(9 \mathrm{~b})$ and $9 \mathrm{gc}$ in the following convenient form:

$$
\begin{aligned}
\partial_{t} C(t, x, \varsigma) & =R_{3}\left(H_{i}, C, N\right)+\nabla \cdot(D(C, N) \nabla C) \\
C(0, x, \varsigma) & =C_{0}(x, \varsigma) \quad \text { for each } \varsigma \in \Sigma_{\epsilon} \\
\nabla C(t, x, \varsigma) \cdot \hat{n} & =0 \quad \text { for each } \varsigma \in \Sigma_{\epsilon}, x \in \partial \Omega \\
\frac{d}{d t} N(t, x, \varsigma) & =R_{4}\left(H_{e}, C, N\right), \quad \text { for each } x \in \Omega, \varsigma \in \Sigma_{\epsilon} \\
N(0, x, \varsigma) & =N_{0}(x, \varsigma)
\end{aligned}
$$


Analogous to the analysis of (SPDM), we first construct a sequence $\left(C^{m}, N^{m}\right)_{m}$ of solutions to

$$
\begin{aligned}
\partial_{t} C^{m} & =R_{3}\left(H_{i}, C^{m-1}, N^{m}\right)+\nabla \cdot\left(D\left(C^{m-1}, N^{m}\right) \nabla C^{m}\right) \quad(m \geq 1) \\
\partial_{t} C^{0} & =R_{3}\left(H_{i}, C_{0}, N^{0}\right)+\nabla \cdot\left(D\left(C_{0}, N^{0}\right) \nabla C^{0}\right) \\
C^{m}(0, x, \varsigma) & =C_{0}(x, \varsigma) \quad \text { for each } x \in \Omega, \varsigma \in \Sigma_{\epsilon} \quad(m \geq 0) \\
\nabla C^{m} \cdot \hat{n} & =0 \quad \text { for each } \varsigma \in \Sigma_{\epsilon}, x \in \partial \Omega \quad(m \geq 0) \\
\frac{d}{d t} N^{m}(t, x, \varsigma) & =R_{4}\left(H_{e}, C^{m-1}, N^{m}\right), \quad(m \geq 1) \quad \text { for each } x \in \Omega, \varsigma \in \Sigma_{\epsilon} \\
\frac{d}{d t} N^{0}(t, x, \varsigma) & =R_{4}\left(H_{e}, C_{0}, N^{0}\right), \quad(m \geq 1) \\
N^{m}(0, x, \varsigma) & =N_{0}(x, \varsigma), \quad(m \geq 0),
\end{aligned}
$$

where we use

\section{Notation 3.}

$$
\begin{aligned}
R_{3}^{m} & :=R_{3}\left(H_{i}, C^{m-1}, N^{m}\right), \quad \forall m \geq 1, \\
R_{3}^{0} & :=R_{3}\left(H_{i}, C_{0}, N^{0}\right), \\
R_{4}^{m} & :=R_{4}\left(H_{e}, C^{m-1}, N^{m}\right), \quad \forall m \geq 0, \\
D^{m} & :=D\left(C^{m-1}, N^{m}\right), \quad \forall m \geq 1 .
\end{aligned}
$$

and then show that the sequence converges in some appropriate sense. This will occupy the rest of the section.

First we easily note that the function $R_{3}\left(H_{i}, C, N\right)$ is an element of $\mathbb{L}_{\Sigma}$ :

Lemma 3.15. Let $0<C(t, x, \varsigma) \leq C_{C}(T)$ and $0<N(t, x, \varsigma) \leq C_{N}$, where $C_{N}$ and $C_{C}(T)$ are as in Theorem 3.16. Then for an open bounded domain $\Omega$ and a finite time $T>0$

$$
\left\|R_{3}\left(H_{i}, C, N\right)\right\|_{\mathbb{L}_{\Sigma}}<C_{R_{3}}|\Omega| T,
$$

where $C_{R_{3}}:=C_{\Lambda_{1}} C_{C}\left(1+\eta_{N} C_{N}+\eta_{C} C_{C} C_{N}\right)$.

Theorem 3.16. For each $\varsigma \in \Sigma_{\epsilon}$ let $\left(H_{i}(\varsigma), H_{e}(\varsigma)\right)$ be the unique weak solution to (SPDM) and let $\Omega \subset \mathbb{R}^{d}$ with $\partial \Omega \in C^{2}$ and $C_{0} \in W^{2, p}(\Omega), N_{0} \in W^{2, p}(\Omega)$ with $p>d+2$ such that the uniform bounds $0<C_{0} \leq M_{C_{0}}$ and $0<N_{0} \leq C_{N}$ exist. Then, $\left(C^{m}(\varsigma)\right)_{m \in \mathbb{N}^{*}} \subset \mathfrak{H}^{1,2} \cap W(p, T, \Omega)$ is the sequence of unique weak solutions to the corresponding m-equations (46a) and 46b), respectively, and $\left(N^{m}(\varsigma)\right)_{m \in \mathbb{N}^{*}} \subset \mathfrak{H}^{1,0}$ is the sequence of unique solutions to the m-equations (47). Moreover, these sequences satisfy the following inequalities:

$$
\begin{aligned}
\left\|N^{m}\right\|_{\mathbb{X}}^{2} & \leq\left(1+C_{\Lambda_{2}}^{2}\right)\left\|N_{0}\right\|_{L^{2}\left(\Sigma_{\epsilon} ; L^{2}(\Omega)\right)}^{2} \quad \forall m \in \mathbb{N} \\
\left\|C^{m}\right\|_{\mathbb{Z}}^{2} & \leq r_{5}\left\|R_{3}^{m}\right\|_{\mathbb{L}_{\Sigma}}^{2}+r_{6}\left\|C_{0}\right\|_{L^{2}\left(\Sigma_{\epsilon} ; H^{1}(\Omega)\right)}^{2} \quad \forall m \in \mathbb{N}
\end{aligned}
$$

with

$$
\begin{array}{lll}
r_{5}:=q_{8}+q_{7}+1, & r_{6}:=q_{9}+\left(q_{7}+1\right) c_{D}, & q_{8}:=q_{5}+\left(q_{6}+1\right) q_{3}, \\
q_{3}=q_{4}:=T e^{\frac{T}{2}}\left(1+\frac{2}{c_{D} \epsilon}\right), & q_{9}:=\left(q_{6}+1\right) q_{4}, & r_{2}:=c_{D}+T e^{\frac{T}{2}}\left(1+\frac{2}{c_{D} \epsilon}\right), \\
r_{3}:=1+T e^{\frac{T}{2}}\left(1+\frac{2}{c_{D} \epsilon}\right), & q_{7}=q_{5}:=\left(\frac{16 C_{f}^{2}}{c_{D}^{2}}\right), & q_{6}:=\left(\max \left\{\frac{c_{D}}{4}, \frac{2 C_{d} C_{f}}{c_{D}}\right\} \frac{4}{c_{D}}\right) \frac{1}{c_{D}} .
\end{array}
$$

Furthermore, if $\hat{T}>0$ is the time satisfying the condition (38) then there exists a time $T$ with

$$
T \leq \hat{T}
$$

such that each $C^{m}(\varsigma) \in\left(C^{m}(\varsigma)\right)_{m \in \mathbb{N}^{*}}$ is uniformly bounded with

$$
\begin{gathered}
0<C^{m}(t, x, \varsigma) \leq C_{C}(T), \quad \forall m \in \mathbb{N}^{*}, t \in[0, T), x \in \Omega, \\
C_{C}(T):=K_{C_{0}}+\exp (-T)-\exp \left(-b_{3} T\right)<\underbrace{K_{C_{0}}+1}_{K_{C}}, 0<K_{C_{0}} \leq 1, b_{3}>0,
\end{gathered}
$$


and each $N^{m}(\varsigma) \in\left(N^{m}(\varsigma)\right)_{m \in \mathbb{N}^{*}}$ is uniformly bounded with

$$
0<N^{m}(t, x, \varsigma) \leq C_{N}, \forall m \in \mathbb{N}^{*}, t \in[0, T), x \in \Omega .
$$

Proof. Due to the iterative coupling of equations 46a (respectively (46b) and (47), we use as before an induction argument to show the existence of a sequence of uniqueness of their solutions. Induction start $m=0$ :

The induction start is verified by performing the following steps:

1. Show that $N^{0}(x, t, \varsigma)$ is uniformly bounded $\forall x \in \Omega, t \in[0, T], \varsigma \in \Sigma_{\epsilon}$.

2. Observe that $N^{0} \in \mathbb{X}$ is the unique solution to (47) for $m=0$.

3. Show that $C^{0}(x, t, \varsigma)$ is uniformly bounded $\forall x \in \Omega, t \in[0, T], \varsigma \in \Sigma_{\epsilon}$.

4. Use 1., 2. and 3. to show that $C^{0} \in \mathbb{Z}$ is the unique solution to 46b).

Note that once the above steps are verified then due to Lemma 3.15 an analogous proof can be applied for the induction step, i.e., assume the claim holds for $m$ and prove it for $m+1$.

For each $x \in \Omega$ and $\varsigma \in \Sigma_{\epsilon}$ we have by (47) for $m=0$ that

$$
N^{0}(t, x, \varsigma)=N_{0}(x, \varsigma) \exp \left(-\int_{0}^{t} \Lambda_{2}\left(H_{e}\right) C_{0}(s, x, \varsigma) d s\right),
$$

from which immediately follows the bound (by the maximum principle $C_{0}$ is nonnegative):

$$
0<N^{0}(t, x, \varsigma) \leq C_{N} \forall \varsigma \in \Sigma_{\epsilon}, x \in \Omega, t \in[0, T],
$$

as $0<N_{0}(x, \varsigma) \leq C_{N}$, and $\Lambda_{2}\left(H_{e}\right) \geq 0 \forall t \in[0, T], x \in \Omega, \varsigma \in \Sigma_{\epsilon}$. It is straightforward to verify that $N^{0}(\varsigma) \in \mathfrak{H}^{1,0}$ for each $\varsigma \in \Sigma_{\epsilon}$ and $N^{0} \in \mathbb{X}$. This completes steps 1 and 2 .

To ensure an upper bound for $C^{0}$, consider equation $46 \mathrm{~b}$ and use the following auxiliary function (like in [28):

$$
\varrho^{0}:=-\exp (-q t)+\exp (-t)+M_{C_{0}}-C^{0} .
$$

Then

$$
\partial_{t} \varrho^{0}-\nabla \cdot\left(D\left(C_{0}, N^{0}\right) \nabla \varrho^{0}\right)=b_{3} \exp \left(-b_{3} t\right)-\exp (-t)-R_{3}^{0} .
$$

Since $C_{0}(x) \leq M_{C_{0}} \forall x \in \Omega$ by construction $\varrho^{0}(0, x) \geq 0 \forall x \in \Omega$. Thus by choosing $q>0$ such that

$$
b_{3} \exp \left(-b_{3} t\right) \geq \exp (-t)+\left|R_{3}^{0}\right|
$$

we can apply the non-negativity theorem in [26] to get that $\varrho^{0}(t, x) \geq 0 \forall x \in \Omega, \forall t \in(0, T)$, $T<\infty$. This in turn implies that $C^{0}(t, x) \leq C_{C}(t)$ with

$$
C_{C}(t):=M_{C_{0}}+\exp (-t)-\exp \left(-b_{3} t\right) \leq \underbrace{M_{C_{0}}+1-\exp \left(-b_{3} T\right)}_{C_{C}(T)}<\underbrace{M_{C_{0}}+1}_{M_{C}} .
$$

Moreover, from the non-negativity theorem in [26] it immediately follows that $C^{0}>0$ for all $x \in \Omega$ and $t \in(0, T)$. This completes step 3 .

For a.e. $\varsigma \in \Sigma_{\epsilon}$, let

$$
C_{n}^{0}(t, x, \varsigma)=\sum_{k=0}^{n} d_{k}(t, \varsigma) w_{k}(x),
$$

where

$$
\left\{w_{k}\right\}_{k \in \mathbb{N}^{*}} \text { is an orthogonal basis of } H^{1}(\Omega)
$$

and

$$
\left\{w_{k}\right\}_{k \in \mathbb{N}^{*}} \text { is also an orthonormal basis of } L^{2}(\Omega)
$$

and $d_{k}(t, \varsigma)$ are chosen such that

$$
d_{k}(0)=\left(C_{0}, w_{k}\right) \quad \text { and } \quad\left(\frac{d}{d t} C_{n}^{0}, w_{k}\right)=\left(R_{3}^{0}, w_{k}\right)-\int_{\Omega} D\left(C_{0}, N^{0}\right) \nabla C_{n}^{0} \nabla w_{k} d x .
$$


Also let us note that, since $C_{0}, N^{0}$ are uniformly bounded, Lemma 3.15 is applicable and hence the reaction term $R_{3}^{0} \in L^{2}\left(0, T ; L^{2}(\Omega)\right)$ for all $\varsigma \in \Sigma_{\epsilon}$.

Now multiply the weak formulation (59) above with $d_{k}$ and sum. This leads to

$$
\frac{1}{2} \frac{d}{d t}\left\|C_{n}^{0}\right\|_{L^{2}(\Omega)}^{2}+c_{D}\left\|\nabla C_{n}^{0}\right\|_{L^{2}(\Omega)}^{2} \leq\left\|R_{3}^{0}\right\|_{L^{2}(\Omega)}\left\|C_{n}^{0}\right\|_{L^{2}(\Omega)},
$$

from which by using Lemma 3.15 and Gronwall's inequality we obtain the bound

$$
\left\|C_{n}^{0}\right\|_{L^{2}(\Omega)}^{2} \leq e^{\frac{T}{2}}\left(\left\|C_{0}\right\|_{L^{2}(\Omega)}^{2}+\frac{1}{2}\left\|R_{3}^{0}\right\|_{\mathbb{L}_{\Omega, T}}^{2}\right) \leq C(T,|\Omega|)\left\|C_{0}\right\|_{L^{2}(\Omega)}^{2},
$$

with $C(T,|\Omega|)=e^{T / 2}\left(1+\frac{1}{2} C_{R_{3}}^{2}|\Omega|^{2} T^{2}\right)$.

For a sufficiently small $\epsilon>0$, we also get that for each $t \in[0, T]$

$$
\frac{1}{2} \frac{d}{d t}\left\|C_{n}^{0}\right\|_{L^{2}(\Omega)}^{2} \leq \frac{1}{\epsilon}\left\|C_{n}^{0}\right\|_{L^{2}(\Omega)}^{2}, \quad \text { hence } \quad\left\|C_{n}^{0}\right\|_{L^{2}(\Omega)}^{2} \leq e^{\frac{2 T}{\epsilon}}\left\|C_{0}\right\|_{L^{2}(\Omega)}^{2}
$$

and

$$
c_{D}\left\|\nabla C_{n}^{0}\right\|_{L^{2}(\Omega)}^{2} \leq \frac{1}{\epsilon}\left\|C_{n}^{0}\right\|_{L^{2}(\Omega)}^{2}-\frac{1}{2} \frac{d}{d t}\left\|C_{n}^{0}\right\|_{L^{2}(\Omega)}^{2}<\infty .
$$

Integrating w.r.t. $t$ leads to

$$
\left\|\nabla C_{n}^{0}\right\|_{\mathbb{L}_{\Omega, T}}^{2} \leq \frac{2}{c_{D} \epsilon}\left\|C_{n}^{0}\right\|_{\mathbb{L}_{\Omega, T}}^{2} .
$$

Altogether, from (61) and (64) we get that

$$
\begin{aligned}
\left\|C_{n}^{0}\right\|_{L^{2}\left(0, T ; H^{1}(\Omega)\right)}^{2} & \leq T e^{\frac{T}{2}}\left(\left\|C_{0}\right\|_{H^{1}(\Omega)}^{2}+\left\|R_{3}^{0}\right\|_{\mathbb{L}_{\Omega, T}}^{2}\right)\left(1+\frac{2}{c_{D} \epsilon}\right) \\
& =q_{3}\left\|R_{3}^{0}\right\|_{\mathbb{\Omega}_{\Omega, T}}^{2}+q_{4}\left\|C_{0}\right\|_{H^{1}(\Omega)}^{2},
\end{aligned}
$$

where $q_{3}:=T e^{\frac{T}{2}}\left(1+\frac{2}{c_{D} \epsilon}\right)$ and $q_{4}:=q_{3}$.

Similarly, multiply the weak formulation $(59)$ by $d_{k}^{\prime}$ and sum to obtain

$$
\begin{aligned}
\left\langle\partial_{t} C_{n}^{0}, \partial_{t} C_{n}^{0}\right\rangle & =\left(R_{3}^{0}, \partial_{t} C_{n}^{0}\right)-\left(D\left(C_{0}, N^{0}\right) \nabla C_{n}^{0}, \nabla \partial_{t} C_{n}^{0}\right) \\
\left\|\partial_{t} C_{n}^{0}\right\|_{L^{2}(\Omega)}^{2}+\frac{1}{2} c_{D} \frac{d}{d t}\left\|\nabla C_{n}^{0}\right\|_{L^{2}(\Omega)}^{2} & \leq \frac{1}{2}\left\|R_{3}^{0}\right\|_{L^{2}(\Omega)}^{2}+\frac{1}{2}\left\|\partial_{t} C_{n}^{0}\right\|_{L^{2}(\Omega)}^{2} \\
\left\|\partial_{t} C_{n}^{0}\right\|_{L^{2}(\Omega)}^{2}+c_{D} \frac{d}{d t}\left\|\nabla C_{n}^{0}\right\|_{L^{2}(\Omega)}^{2} & \leq\left\|R_{3}^{0}\right\|_{L^{2}(\Omega)}^{2}
\end{aligned}
$$

from which by integration w.r.t. $t$ we get

$$
\left\|\partial_{t} C_{n}^{0}\right\|_{\mathbb{L}_{\Omega, T}}^{2}+c_{D}\left\|\nabla C_{n}^{0}\right\|_{L^{2}(\Omega)}^{2} \leq\left\|R_{3}^{0}\right\|_{\mathbb{L}_{\Omega, T}}^{2}+c_{D}\left\|C_{0}\right\|_{H^{1}(\Omega)}^{2} .
$$

Hence from 61, 65), 68) we obtain that

$$
\begin{aligned}
\max _{0 \leq t \leq T}\left\|C_{n}^{0}(t)\right\|_{L^{2}(\Omega)}^{2} & \leq e^{T / 2}\left(\left\|C_{0}\right\|_{H^{1}(\Omega)}^{2}+\frac{1}{2}\left\|R_{3}^{0}\right\|_{\mathbb{L}_{\Omega, T}}^{2}\right)<\infty \\
\left\|C_{n}^{0}\right\|_{L^{2}\left(0, T ; H^{1}(\Omega)\right)}^{2} & \leq q_{3}\left\|R_{3}^{0}\right\|_{\mathbb{L}_{\Omega, T}}^{2}+q_{4}\left\|C_{0}\right\|_{H^{1}(\Omega)}^{2}<\infty \\
\left\|\partial_{t} C_{n}^{0}\right\|_{\mathbb{L}_{\Omega, T}}^{2} & \leq\left\|R_{3}^{0}\right\|_{\mathbb{L}_{\Omega, T}}^{2}+c_{D}\left\|C_{0}\right\|_{H^{1}(\Omega)}^{2}<\infty \\
\max _{0 \leq t \leq T}\left\|\nabla C_{n}^{0}(t)\right\|_{L^{2}(\Omega)}^{2} & \leq \frac{1}{c_{D}}\left(\left\|R_{3}^{0}\right\|_{\mathbb{L}_{\Omega, T}}^{2}+c_{D}\left\|C_{0}\right\|_{H^{1}(\Omega)}^{2}\right)<\infty
\end{aligned}
$$

Hence there exist some subsequences $\left(C_{m_{k}}^{0}\right)_{k}$ and $\left(\partial_{t} C_{m_{k}}^{0}\right)_{k}$ which converge weakly to $C^{0} \in L^{2}\left(0, T ; H^{1}(\Omega)\right)$ and $\left(C^{0}\right)^{\prime} \in L^{2}\left(0, T ; L^{2}(\Omega)\right)$; it is easy to see that $\left(C^{0}\right)^{\prime}=\partial_{t} C^{0}$.

Altogether we have with the usual passage to limits in the corresponding weak formulations

$$
\begin{aligned}
\left\|C^{0}\right\|_{L^{2}\left(0, T ; H^{1}(\Omega)\right)}^{2}+\left\|\partial_{t} C^{0}\right\|_{\mathbb{L}_{\Omega, T}}^{2} & \leq\left(c_{D}+q_{4}\right)\left\|C_{0}\right\|_{H^{1}(\Omega)}^{2}+\left(1+q_{3}\right)\left\|R_{3}^{0}\right\|_{\mathbb{L}_{\Omega, T}}^{2} \\
& \leq r_{3}\left\|R_{3}^{0}\right\|_{\mathbb{L}_{\Omega, T}}^{2}+r_{2}\left\|C_{0}\right\|_{H^{1}(\Omega)}^{2}
\end{aligned}
$$


from which by taking the $\mathbb{E}_{\varepsilon}$-expectation we get

$$
\left\|C^{0}\right\|_{\mathbb{Y}}^{2} \leq r_{3}\left\|R_{3}^{0}\right\|_{\mathbb{L}_{\Sigma}}^{2}+r_{2}\left\|C_{0}\right\|_{H^{1}(\Omega)}^{2}<\infty
$$

where $r_{3}:=1+q_{3}=1+T e^{\frac{T}{2}}\left(1+\frac{2}{c_{D} \epsilon}\right)$ and $r_{2}:=c_{D}+q_{4}=c_{D}+T e^{\frac{T}{2}}\left(1+\frac{2}{c_{D} \epsilon}\right)$.

Remark 4. From the hypothesis we have that $\Omega \subset \mathbb{R}^{d}, \partial \Omega \in C^{2}$ and $C_{0}, N_{0} \in W^{2, p}(\Omega) \hookrightarrow C^{1}(\bar{\Omega})$ for $p>d+2$. Also by Remark 3 we have that $H_{e} \in W^{2, p}(\Omega)$, hence $N^{0}(t) \in W^{2, p}(\Omega)$. Thus we get that $D\left(C_{0}, N^{0}(t)\right) \in W^{2, p}(\Omega)$ for each $t \in[0, T)$ and a.e. $\varsigma \in \Sigma_{\epsilon}$. This in turn implies that

$$
D\left(C_{0}, N^{0}\right) \in C^{1}([0, T] \times \bar{\Omega}), \quad \nabla D\left(C_{0}, N^{0}\right) \in C([0, T] \times \bar{\Omega}) .
$$

Since $D\left(C_{0}, N^{0}\right) \in W(p, T, \Omega) \hookrightarrow L^{2 p}\left(0, T ; W^{1,2 p}(\Omega)\right)$ and $\nabla D^{0} \in L^{2 p}\left(0, T ; L^{2 p}(\Omega)\right)$ (Lemma 3.3, Chapter 2 of [25]) we can again apply Theorem 9.1 (Chapter 4 of [25]) to get that $C^{0} \in W(p, T, \Omega)$. We use this to claim the continuity $\nabla D\left(C^{0}, N^{1}\right)$. Thus, the induction procedure yields the uniform continuity of $\nabla D\left(C^{m-1}, N^{m}\right)$.

For the convergence of the sequence $\left(C^{m}\right)_{m \in \mathbb{N}^{*}}$ we use again the compact embedding $W(p, T, \Omega) \hookrightarrow \hookrightarrow L^{p}\left(0, T ; L^{p}(\Omega)\right) \hookrightarrow L^{2}\left(0, T ; L^{2}(\Omega)\right)$ (see Remark 3 ) and get that the subsequence $\left(C^{m_{j}}\right)_{j \in \mathbb{N}}$ converging weakly in $W(p, T, \Omega)$ also converges strongly in $L^{p}\left(0, T ; L^{p}(\Omega)\right)$ and $L^{2}\left(0, T ; L^{2}(\Omega)\right)$. However, for the ease of notation we ignore the subscript $j$ and still refer the subsequence $\left(C^{m_{j}}\right)_{j \in \mathbb{N}}$ as $\left(C^{m}\right)_{m \in \mathbb{N}^{*}}$.

By the regularity theorem (Theorem 7.1.5) in [6] we get the following estimate for the $H^{2}$ norm:

$$
\begin{aligned}
&\left\|\Delta C^{0}\right\|_{\mathbb{L}_{\Omega, T}}^{2} \leq q_{5}\left\|R_{3}^{0}\right\|_{\mathbb{L}_{\Omega, T}}^{2}+q_{6}\left\|C^{0}\right\|_{L^{2}\left(0, T ; H^{1}(\Omega)\right)}^{2}+q_{7}\left\|\partial_{t} C^{0}(\varsigma)\right\|_{\mathbb{L}_{\Omega, T}}^{2} \\
&\left\|C^{0}\right\|_{L^{2}\left(0, T ; H^{1}(\Omega)\right)}^{2}+\left\|\Delta C^{0}\right\|_{\mathbb{L}_{\Omega, T}}^{2} \leq q_{8}\left\|R_{3}^{0}\right\|_{\mathbb{L}_{\Omega, T}}^{2}+q_{9}\left\|C_{0}\right\|_{H^{1}(\Omega)}^{2}+q_{7}\left\|\partial_{t} C^{0}\right\|_{\mathbb{L}_{\Omega, T}}^{2} \\
&\left\|C^{0}\right\|_{L^{2}\left(0, T ; H^{2}(\Omega)\right)}^{2}+\left\|\partial_{t} C^{0}\right\|_{\mathbb{L}_{\Omega, T}}^{2} \leq r_{5}\left\|R_{3}^{0}\right\|_{\mathbb{L}_{\Omega, T}}^{2}+r_{6}\left\|C_{0}\right\|_{H^{1}(\Omega)}^{2}
\end{aligned}
$$

where

$$
\begin{array}{lll}
q_{7}=q_{5}:=\left(\frac{16 C_{f}^{2}}{c_{D}^{2}}\right), & q_{6}:=\left(\max \left\{\frac{c_{D}}{4}, \frac{2 C_{d} C_{f}}{c_{D}}\right\} \frac{4}{c_{D}}\right), & q_{8}:=q_{5}+\left(q_{6}+1\right) q_{3}, \\
q_{9}:=\left(q_{6}+1\right) q_{4}, & r_{5}:=q_{8}+q_{7}+1, & r_{6}:=q_{9}+\left(q_{7}+1\right) c_{D} .
\end{array}
$$

This completes the induction start $m=0$.

Induction step $m$ : Assume the claim holds for the $m$ th term in the sequence and show that this also implies the claim for the $m+1$ st term.

The proof is exactly the same as for induction start, therefore it holds that $C^{m} \in \mathbb{Z}$ and $N^{m} \in \mathbb{X}$ are the unique solution sequence to the corresponding $m$-th equations 46a) and (47), respectively.

\subsubsection{Existence and uniqueness of the solution to (CPDM)}

We consider the sequence $\left(C^{m}\right)_{m \in \mathbb{N}^{*}} \subset \mathbb{Z}$, such that for fixed $\varsigma \in \Sigma_{\epsilon}$, the sequence $\left(C^{m}(\varsigma)\right)_{m \in \mathbb{N}^{*}} \subset \mathfrak{H}^{1,2}$ contains the solutions to the corresponding $m$-th equation specified by (46a). Similarly, we take $\left(N^{m}\right)_{m \in \mathbb{N}^{*}} \subset \mathbb{X}$, such that for fixed $\varsigma \in \Sigma_{\epsilon}$, the sequence $\left(N^{m}(\varsigma)\right)_{m \in \mathbb{N}^{*}} \subset \mathfrak{H}^{1,0}$ features the solutions to the corresponding $m$-th equation specified by (47).

In order to prove the existence of a solution we need to show that the sequences $\left(C^{m}\right)_{m}$ and $\left(N^{m}\right)_{m}$ converge in $\mathbb{Z}$ and $\mathbb{X}$, respectively. To this end let us collect some inequalities. The following lemma is easily verified:

Lemma 3.17. Let $C^{n}, C^{m} \in\left(C^{m}\right)_{m}$ and $N^{n}, N^{m} \in\left(N^{m}\right)_{m}$ be some arbitrary elements of the respective solution sequences. Then the following inequalities hold:

$$
\left\|R_{3}^{m}-R_{3}^{n}\right\|_{\mathbb{L}_{\Sigma}}^{2} \leq Q_{C_{1}}\left\|C^{m-1}-C^{n-1}\right\|_{\mathbb{L}_{\Sigma}}^{2}+Q_{C_{2}}\left\|N^{m}-N^{n}\right\|_{\mathbb{L}_{\Sigma}}^{2},
$$

with $Q_{C_{1}}:=2 C_{\Lambda_{1}}^{2}\left(1+2 \eta_{C} C_{C}+\eta_{N} C_{N}\right)^{2}$ and $Q_{C_{2}}:=2 C_{\Lambda_{1}}^{2}\left(\eta_{N} C_{C}\right)^{2}$.

$$
\left\|R_{4}^{m}-R_{4}^{n}\right\|_{\mathbb{L}_{\Sigma}}^{2} \leq 2 C_{\Lambda_{2}}^{2}\left(C_{C}^{2}\left\|N^{m}-N^{n}\right\|_{\mathbb{L}_{\Sigma}}^{2}+C_{N}^{2}\left\|C^{m-1}-C^{n-1}\right\|_{\mathbb{L}_{\Sigma}}^{2}\right)
$$


Now we prove a sufficient condition for the sequence $\left(N^{m}\right)_{m}$ to converge in $\mathbb{X}$.

Lemma 3.18. Let $C^{m}(\varsigma), C^{n}(\varsigma) \in \mathfrak{H}^{1,2}$ and $N^{m}(\varsigma), N^{n}(\varsigma) \in \mathfrak{H}^{1,0}$ be arbitrary elements of the solution sequences $\left(C^{m}\right)_{m}$ and $\left(N^{m}\right)_{m}$, respectively. Then $\left(N^{m}\right)_{m}$ converges in $\mathbb{X}$ if and only if $\left(C^{m}\right)_{m}$ converges in $\mathbb{L}_{\Sigma}$. Moreover, the difference $\left\|N^{m}-N^{n}\right\|_{\mathbb{X}}$ satisfies the following inequality:

$$
\left\|N^{m}-N^{n}\right\|_{\mathbb{X}}^{2} \leq Q_{N_{1}}\left(1+Q_{N_{2}}\right)+Q_{N_{3}}\left\|C^{m-1}-C^{n-1}\right\|_{\mathbb{L}_{\Sigma}}^{2},
$$

with $Q_{N_{1}}:=T e^{2 C_{\Lambda_{2}} C_{C} T} C_{\Lambda_{2}}^{4} C_{C}^{2} C_{N}^{2}, Q_{N_{2}}:=2 C_{\Lambda_{2}}^{2} C_{C}^{2}$ and $Q_{N_{3}}:=2 C_{\Lambda_{2}}^{2} C_{N}^{2}$.

Proof. From 477) we get

$$
\left|N^{m}(t)-N^{n}(t)\right| \leq C_{\Lambda_{2}} C_{C} \int_{0}^{t}\left|N^{m}(s)-N^{n}(s)\right| d s+C_{\Lambda_{2}} C_{N} \int_{0}^{T}\left|C^{m-1}(s)-C^{n-1}(s)\right| d s,
$$

from which by Gronwall's inequality

$$
\left|N^{m}(t)-N^{n}(t)\right| \leq e^{C_{\Lambda_{2}} C_{C} T} C_{\Lambda_{2}}^{2} C_{C} C_{N} \int_{0}^{T}\left|C^{m-1}(s)-C^{n-1}(s)\right| d s .
$$

Thus,

$$
\begin{aligned}
\left\|N^{m}(t)-N^{n}(t)\right\|_{L^{2}(\Omega)}^{2} & \leq e^{2 C_{\Lambda_{2}} C_{C} T}\left(C_{\Lambda_{2}}^{4} C_{C}^{2} C_{N}^{2}\right)\left\|C^{m-1}-C^{n-1}\right\|_{\mathbb{L}_{\Omega, T}}^{2} \\
\left\|N^{m}-N^{n}\right\|_{\mathbb{L}_{\Omega, T}}^{2} & \leq Q_{N_{1}}\left\|C^{m-1}-C^{n-1}\right\|_{\mathbb{L}_{\Omega, T}}^{2}
\end{aligned}
$$

Again from (47) we have that

$$
\left.\left|\partial_{t}\left(N^{m}(t)-N^{n}(t)\right)\right| \leq C_{\Lambda_{2}} C_{C} \mid N^{m}(t)-N^{n}(t)\right)\left|+C_{\Lambda_{2}} C_{N}\right| C^{m-1}(t)-C^{n-1}(t) \mid
$$

hence

$$
\left\|\partial_{t}\left(N^{m}\right)-\partial_{t}\left(N^{n}\right)\right\|_{\mathbb{L}_{\Omega, T}}^{2} \leq 2\left(C_{\Lambda_{2}} C_{C}\right)^{2}\left\|N^{m}-N^{n}\right\|_{\mathbb{L}_{\Omega, T}}^{2}+2\left(C_{\Lambda_{2}} C_{N}\right)^{2}\left\|C^{m-1}-C^{n-1}\right\|_{\mathbb{L}_{\Omega, T}}^{2} .
$$

Thus from (76) and 777 we have that

$$
\begin{aligned}
\left\|N^{m}-N^{n}\right\|_{\mathbb{L}_{\Omega, T}}^{2}+\left\|\partial_{t} N^{m}-\partial_{t} N^{n}\right\|_{\mathbb{L}_{\Omega, T}}^{2} \leq Q_{N_{1}}(1+ & \left.2 C_{\Lambda_{2}}^{2} C_{C}^{2}\right)\left\|C^{m-1}-C^{n-1}\right\|_{\mathbb{L}_{\Omega, T}}^{2} \\
& +2 C_{\Lambda_{2}}^{2} C_{N}^{2}\left\|C^{m-1}-C^{n-1}\right\|_{\mathbb{L}_{\Omega, T}}^{2}
\end{aligned}
$$

and taking the $\mathbb{E}_{\varepsilon}$-expectation we arrive at

$$
\left\|N^{m}-N^{n}\right\|_{\mathbb{X}}^{2} \leq\left(Q_{N_{1}}\left(1+Q_{N_{2}}\right)+Q_{N_{3}}\right)\left\|C^{m-1}-C^{n-1}\right\|_{\mathbb{L}_{\Sigma}}^{2} .
$$

Similarly, using the inequality $(53)$ from Theorem 3.16 we get the following sufficient condition for the convergence of $\left(C^{m}\right)_{m} \subset \mathbb{Z}$.

Lemma 3.19. Let $C^{m}(\varsigma), C^{n}(\varsigma) \in \mathfrak{H}^{1,2}$ and $N^{m}(\varsigma), N^{n}(\varsigma) \in \mathfrak{H}^{1,0}$ be arbitrary elements of the respective solution sequences, then $\left(C^{m}\right)_{m}$ converges in $\mathbb{Z}$ if and only if $\left(C^{m}\right)_{m}$ converges in $\mathbb{L}_{\Sigma}$, because the difference $\left\|C^{m}-C^{n}\right\|$ satisfies the following inequality

$$
\left\|C^{m}-C^{n}\right\|_{\mathbb{Z}}^{2} \leq Q_{C_{11}}\left\|C^{m-1}-C^{n-1}\right\|_{\mathbb{L}_{\Sigma}}^{2}+Q_{C_{12}}\left\|C^{m-1}-C^{n-1}\right\|_{\mathbb{L}_{\Sigma}},
$$

with

$$
\begin{array}{ll}
Q_{C_{3}}:=Q_{C_{1}}+Q_{C_{2}} Q_{N_{1}}, & Q_{C_{4}}:=r C_{C} \sqrt{Q_{N_{1}}}+a_{1} \tilde{C}_{N} \\
Q_{C_{5}}:=\frac{Q_{C_{1}}}{c_{D}}+Q_{N_{1}} \frac{Q_{C_{2}}}{c_{D}}+\frac{T e^{T} Q_{C_{3}}}{c_{D}}, & Q_{C_{6}}:=\frac{T e^{T} Q_{C_{4}}}{c_{D}}+\frac{a_{1} \tilde{C}_{C}}{c_{D}}+\frac{a_{1} \tilde{C}_{N}}{c_{D}} \sqrt{Q_{N_{1}}} \\
Q_{C_{7}}:=4 Q_{C_{1}}^{2}+4 Q_{N_{1}}\left(Q_{C_{2}}^{2}+4 a_{1}^{2} \tilde{C}_{C}^{2}\right)+4 a_{1}^{2} \tilde{C}^{N}, & Q_{C_{8}}:=4 C_{D}^{2} \\
Q_{C_{9}}:=q_{5} Q_{C_{1}}^{2}+q_{7} Q_{C_{7}}+q_{7} Q_{C_{8}} Q_{C_{5}}+q_{5} Q_{C_{2}}^{2} Q_{N_{1}}, & Q_{C_{10}}:=q_{7} Q_{C_{8}} Q_{C_{6}} \\
Q_{C_{11}}:=T e^{T} Q_{C_{3}}+\left(Q_{C_{8}}+1\right) Q_{C_{5}}+Q_{C_{7}}+Q_{C_{9}}, & Q_{C_{12}}:=T e^{T} Q_{C_{4}}+Q_{C_{6}}\left(Q_{C_{8}}+1\right)+Q_{C_{10}}
\end{array}
$$

and $a_{1}$ such that

$$
8\left(\frac{1}{c_{D}}+r_{5}\right) C_{R_{3}}^{4}|\Omega|^{4} T+\left(1+r_{6}\right)\left\|C_{0}\right\|_{H^{1}(\Omega)}^{4}<a_{1}<\infty .
$$


Proof. Let $C^{m}(\varsigma), C^{n}(\varsigma) \in\left(C^{m}(\varsigma)\right)_{m}$; then by Theorem $3.16 C^{m}(\varsigma), C^{n}(\varsigma) \in \mathfrak{H}^{1,2}$. In particular, $C^{m}(t, ., \varsigma) \in H^{2}(\Omega)$ for any fixed $t \in[0, T]$ and $m \in \mathbb{N}^{*}$. Consequently, we can set $v:=C^{m}(t, ., \varsigma)-C^{n}(t, ., \varsigma)$ in $46 a$, from which we get for fixed $t \in[0, T]$ and $\varsigma \in \Sigma_{\epsilon}$ (remember Notation 3 :

$$
\begin{aligned}
&\left\langle\partial_{t}\left(C^{m}-C^{n}\right), C^{m}-C^{n}\right\rangle=\left(R_{3}^{m}-R_{3}^{n}, C^{m}-C^{n}\right)-\left(D^{m} \nabla C^{m}-D^{n} \nabla C^{n}, \nabla\left(C^{m}-C^{n}\right)\right) \\
& \frac{1}{2} \frac{d}{d t}\left\|C^{m}-C^{n}\right\|_{L^{2}(\Omega)}^{2}=\left(R_{3}^{m}-R_{3}^{n}, C^{m}-C^{n}\right)-\left(D^{m} \nabla C^{m}-D^{n} \nabla C^{n}, \nabla\left(C^{m}-C^{n}\right)\right) \\
&=\left(R_{3}^{m}-R_{3}^{n}, C^{m}-C^{n}\right)-D^{n}\left(\nabla\left(C^{m}-C^{n}\right), \nabla\left(C^{m}-C^{n}\right)\right) \\
&-\nabla C^{m}\left(D^{m}-D^{n}, \nabla\left(C^{m}-C^{n}\right)\right) \\
& \leq\left\|R_{3}^{m}-R_{3}^{n}\right\|_{L^{2}(\Omega)}\left\|C^{m}-C^{n}\right\|_{L^{2}(\Omega)}-c_{D}\left\|\nabla\left(C^{m}-C^{n}\right)\right\|_{L^{2}(\Omega)}^{2} \\
&+\left\|D^{m}-D^{n}\right\|_{L^{2}(\Omega)}\left\|\nabla C^{m}\right\|_{L^{4}(\Omega)}^{2}\left\|\nabla C^{m}-\nabla C^{n}\right\|_{L^{4}(\Omega)}^{2}
\end{aligned}
$$

Since $C^{m}, C^{n} \in H^{2}(\Omega)$ it implies that $\nabla C^{m}, \nabla C^{n} \in H^{1}(\Omega)$ and we obtain

$$
\begin{aligned}
\frac{1}{2} \frac{d}{d t}\left\|C^{m}-C^{n}\right\|_{L^{2}(\Omega)}^{2}+c_{D} \| \nabla\left(C^{m}\right. & \left.-C^{n}\right)\left\|_{L^{2}(\Omega)}^{2} \leq\right\| R_{3}^{m}-R_{3}^{n}\left\|_{L^{2}(\Omega)}\right\| C^{m}-C^{n} \|_{L^{2}(\Omega)} \\
& +\tilde{C}\left\|D^{m}-D^{n}\right\|_{L^{2}(\Omega)}\left\|\nabla C^{m}\right\|_{H^{1}(\Omega)}^{2}\left\|\nabla C^{m}-\nabla C^{n}\right\|_{H^{1}(\Omega)}^{2},
\end{aligned}
$$

Using the Lipschitz continuity of $R_{3}$ and $D$ we get

$$
\begin{aligned}
\frac{d}{d t}\left\|C^{m}-C^{n}\right\|_{L^{2}(\Omega)}^{2} \leq & \left\|R_{3}^{m}-R_{3}^{n}\right\|_{L^{2}(\Omega)}^{2} \\
& \left\|C^{m}-C^{n}\right\|_{L^{2}(\Omega)}^{2}+a_{1} \tilde{C}_{C}\left\|N^{n}-N^{m}\right\|_{L^{2}(\Omega)} \\
& +a_{1} \tilde{C}_{N}\left\|C^{n-1}-C^{m-1}\right\|_{L^{2}(\Omega)} \\
\leq & Q_{C_{1}}\left\|C^{m-1}-C^{n-1}\right\|_{L^{2}(\Omega)}^{2}+Q_{C_{2}}\left\|N^{m}-N^{n}\right\|_{L^{2}(\Omega)}^{2}+\left\|C^{m}-C^{n}\right\|_{L^{2}(\Omega)}^{2} \\
& +a_{1} \tilde{C}_{C}\left\|N^{n}-N^{m}\right\|_{L^{2}(\Omega)}+a_{1} \tilde{C}_{N}\left\|C^{n-1}-C^{m-1}\right\|_{L^{2}(\Omega)}
\end{aligned}
$$

with $a_{1}$ such that

$$
\begin{aligned}
a & :=\sup _{t \in[0, T)} 4\left\|\nabla C^{m}(t)\right\|_{H^{1}(\Omega)}^{4} \leq \sup _{t \in[0, T)} 8\left\|\nabla C^{m}(t)\right\|_{L^{2}(\Omega)}^{4}+\left\|\Delta C^{m}(t)\right\|_{L^{2}(\Omega)}^{4} \\
& \leq 8\left(\frac{1}{c_{D}}+r_{5}\right)\left\|R_{3}^{m}\right\|_{\mathbb{L}_{\Omega, T}}^{4}+\left(1+r_{6}\right)\left\|C_{0}\right\|_{H^{1}(\Omega)}^{4} \stackrel{49}{\leq} 8\left(\frac{1}{c_{D}}+r_{5}\right) C_{R_{3}}^{4}|\Omega|^{4} T+\left(1+r_{6}\right)\left\|C_{0}\right\|_{H^{1}(\Omega)}^{4}<a_{1}<\infty .
\end{aligned}
$$

Applying Gronwall's inequality and integrating with respect to time we get

$$
\begin{aligned}
\left\|C^{m}-C^{n}\right\|_{\mathbb{L}_{\Omega, T}}^{2} \leq T e^{T}\left(Q_{C_{1}}\left\|C^{m-1}-C^{n-1}\right\|_{\mathbb{L}_{\Omega, T}}^{2}+Q_{C_{2}}\left\|N^{m}-N^{n}\right\|_{\mathbb{L}_{\Omega, T}}^{2}\right. \\
\left.+a_{1} \tilde{C}_{C}\left\|N^{n}-N^{n}\right\|_{\mathbb{L}_{\Omega, T}}+a_{1} \tilde{C}_{N}\left\|C^{m-1}-C^{n-1}\right\|_{\mathbb{L}_{\Omega, T}}\right) .
\end{aligned}
$$

Using 76, we get

$$
\left\|C^{m}-C^{n}\right\|_{\mathbb{L}_{\Omega, T}}^{2} \leq T e^{T}\left(Q_{C_{3}}\left\|C^{m-1}-C^{n-1}\right\|_{\mathbb{L}_{\Omega, T}}^{2}+Q_{C_{4}}\left\|C^{m-1}-C^{n-1}\right\|_{\mathbb{L}_{\Omega, T}}\right),
$$

where $Q_{C_{3}}:=Q_{C_{1}}+Q_{C_{2}} Q_{N_{1}}, Q_{C_{4}}:=a_{1} \tilde{C}_{C} \sqrt{Q_{N_{1}}}+a_{1} \tilde{C}_{N}, \tilde{C}_{C}:=\tilde{C} C_{C}$ and $\tilde{C}_{N}:=\tilde{C} C_{N}$.

From 80 we also get

$$
\begin{gathered}
\left\|\nabla\left(C^{m}-C^{n}\right)\right\|_{\mathbb{L}_{\Omega, T}}^{2} \leq \frac{Q_{C_{1}}}{c_{D}}\left\|C^{m-1}-C^{n-1}\right\|_{\mathbb{L}_{\Omega, T}}^{2}+\frac{Q_{C_{2}}}{c_{D}}\left\|N^{m}-N^{n}\right\|_{\mathbb{L}_{\Omega, T}}^{2}+\frac{1}{c_{D}}\left\|C^{m}-C^{n}\right\|_{\mathbb{L}_{\Omega, T}}^{2} \\
+\frac{a_{1} \tilde{C}_{C}}{c_{D}}\left\|C^{m-1}-C^{n-1}\right\|_{\mathbb{L}_{\Omega, T}}+\frac{a_{1} \tilde{C}_{N}}{c_{D}}\left\|N^{m}-N^{n}\right\|_{\mathbb{L}_{\Omega, T}} .
\end{gathered}
$$

Using (76) we get

$$
\left\|\nabla\left(C^{m}-C^{n}\right)\right\|_{\mathbb{L}_{\Omega, T}}^{2} \leq Q_{C_{5}}\left\|C^{m-1}-C^{n-1}\right\|_{\mathbb{L}_{\Omega, T}}^{2}+Q_{C_{6}}\left\|C^{m-1}-C^{n-1}\right\|_{\mathbb{L}_{\Omega, T}}
$$


where $Q_{C_{5}}:=\frac{Q_{C_{1}}}{c_{D}}+Q_{N_{1}} \frac{Q_{C_{2}}}{c_{D}}+\frac{T e^{T} Q_{C_{3}}}{c_{D}}$ and $Q_{C_{6}}:=\frac{T e^{T} Q_{C_{4}}}{c_{D}}+\frac{a_{1} \tilde{C}_{C}}{c_{D}}+\frac{a_{1} \tilde{C}_{N}}{c_{D}} \sqrt{Q_{N_{1}}}$.

Now for all $v \in H^{1}(\Omega)$ with $\|v\|_{H^{1}(\Omega)} \leq 1$ we have that

$$
\begin{gathered}
\sup _{v}\left|\left\langle\partial_{t}\left(C^{m}-C^{n}\right), v\right\rangle\right| \leq\left\|R_{3}^{m}-R_{3}^{n}\right\|_{L^{2}(\Omega)}\|v\|_{L^{2}(\Omega)}+C_{D}\left\|\nabla C^{m}-\nabla C^{n}\right\|_{L^{2}(\Omega)}\|v\|_{L^{2}(\Omega)} \\
+\left\|D^{m}-D^{n}\right\|_{L^{2}(\Omega)}\left\|\nabla C^{m}\right\|_{L^{4}(\Omega)}\|v\|_{L^{4}(\Omega)} \\
\Rightarrow\left\|\partial_{t}\left(C^{m}-C^{n}\right)\right\|_{\left(H^{1}(\Omega)\right)^{*}} \leq\left\|R_{3}^{m}-R_{3}^{n}\right\|_{L^{2}(\Omega)}\|v\|_{H^{1}(\Omega)}+C_{D}\left\|\nabla C^{m}-\nabla C^{n}\right\|_{L^{2}(\Omega)}\|v\|_{H^{1}(\Omega)} \\
+\tilde{C}\left\|D^{m}-D^{n}\right\|_{L^{2}(\Omega)}\left\|\nabla C^{m}\right\|_{H^{1}(\Omega)}\|v\|_{H^{1}(\Omega)} \\
\leq\left\|R_{3}^{m}-R_{3}^{n}\right\|_{L^{2}(\Omega)}+C_{D}\left\|\nabla C^{m}-\nabla C^{n}\right\|_{L^{2}(\Omega)}+a_{1} \tilde{C}\left\|D^{m}-D^{n}\right\|_{L^{2}(\Omega)}
\end{gathered}
$$

$\stackrel{\int_{0}^{T}}{\Rightarrow}\left\|\partial_{t}\left(C^{m}-C^{n}\right)\right\|_{L^{2}\left(0, T ;\left(H^{1}(\Omega)\right)^{*}\right)} \leq\left\|R_{3}^{m}-R_{3}^{n}\right\|_{\mathbb{L}_{\Omega, T}}+C_{D}\left\|\nabla C^{m}-\nabla C^{n}\right\|_{\mathbb{L}_{\Omega, T}}+a_{1} \tilde{C}\left\|D^{m}-D^{n}\right\|_{\mathbb{L}_{\Omega, T}}$

Using the Lipschitz continuity of $R_{3}$ and $D$ we get that

$$
\begin{gathered}
\left\|\partial_{t}\left(C^{m}-C^{n}\right)\right\|_{L^{2}\left(0, T ;\left(H^{1}(\Omega)\right)^{*}\right)}^{2} \leq 4 Q_{C_{1}}^{2}\left\|C^{m-1}-C^{n-1}\right\|_{\mathbb{L}_{\Omega, T}}^{2}+4 Q_{C_{2}}^{2}\left\|N^{m}-N^{n}\right\|_{\mathbb{L}_{\Omega, T}}^{2}+4 C_{D}^{2}\left\|\nabla\left(C^{m}-C^{n}\right)\right\|_{\mathbb{L}_{\Omega, T}}^{2} \\
4 a_{1}^{2} \tilde{C}_{C}^{2}\left\|N^{m}-N^{n}\right\|_{\mathbb{L}_{\Omega, T}}^{2}+4 a_{1}^{2} \tilde{C}_{N}^{2}\left\|C^{m-1}-C^{n-1}\right\|_{\mathbb{L}_{\Omega, T}}^{2}
\end{gathered}
$$

Using (76) we get

$$
\left\|\partial_{t}\left(C^{m}-C^{n}\right)\right\|_{L^{2}\left(0, T ;\left(H^{1}(\Omega)\right)^{*}\right)}^{2} \leq Q_{C_{7}}\left\|C^{m-1}-C^{n-1}\right\|_{\mathbb{L}_{\Omega, T}}^{2}+Q_{C_{8}}\left\|\nabla\left(C^{m}-C^{n}\right)\right\|_{\mathbb{L}_{\Omega, T}}^{2}
$$

where $Q_{C_{7}}:=4 Q_{C_{1}}^{2}+4 Q_{N_{1}}\left(Q_{C_{2}}^{2}+4 a_{1}^{2} \tilde{C}_{C}^{2}\right)+4 a_{1}^{2} \tilde{C}_{N}^{2}, Q_{C_{8}}:=4 C_{D}^{2}$.

For the second weak derivative we have that

$$
\begin{aligned}
\left\|\Delta\left(C^{m}-C^{n}\right)\right\|_{\mathbb{L}_{\Omega, T}}^{2} & \leq q_{5}\left\|R_{3}^{m}-R_{3}^{n}\right\|_{\mathbb{L}_{\Omega, T}}^{2}+q_{7}\left\|\partial_{t}\left(C^{m}-C^{n}\right)\right\|_{\mathbb{L}_{\Omega, T}}^{2} \\
\leq & \left.q_{5}\left\|R_{3}^{m}-R_{3}^{n}\right\|_{\mathbb{L}_{\Omega, T}}^{2}+q_{7}\left\|\partial_{t}\left(C^{m}-C^{n}\right)\right\|_{L^{2}\left(0, T ;\left(H^{1}(\Omega)\right)^{*}\right.}^{2}\right) \\
& \stackrel{855}{\leq}\left(q_{5} Q_{C_{1}}^{2}+q_{7} Q_{C_{7}}\right)\left\|C^{m-1}-C^{n-1}\right\|_{\mathbb{L}_{\Omega, T}}^{2}+q_{5} Q_{C_{2}}^{2}\left\|N^{m}-N^{n}\right\|_{\mathbb{L}_{\Omega, T}}^{2}+q_{7} Q_{C_{8}}\left\|\nabla\left(C^{m}-C^{n}\right)\right\|_{\mathbb{L}_{\Omega, T}}^{2} \\
& \stackrel{827}{\leq}\left(q_{5} Q_{C_{1}}^{2}+q_{7} Q_{C_{7}}+q_{7} Q_{C_{8}} Q_{C_{5}}\right)\left\|C^{m-1}-C^{n-1}\right\|_{\mathbb{L}_{\Omega, T}}^{2}+q_{7} Q_{C_{8}} Q_{C_{6}}\left\|C^{m-1}-C^{n-1}\right\|_{\mathbb{L}_{\Omega, T}} \\
& \quad+q_{5} Q_{C_{2}}^{2}\left\|N^{m}-N^{n}\right\|_{\mathbb{L}_{\Omega, T}}^{2} \\
& \stackrel{766}{\leq} Q_{C_{9}}\left\|C^{m-1}-C^{n-1}\right\|_{\mathbb{L}_{\Omega, T}}^{2}+Q_{C_{10}}\left\|C^{m-1}-C^{n-1}\right\|_{\mathbb{L}_{\Omega, T}}
\end{aligned}
$$

where $Q_{C_{9}}:=\left(q_{5} Q_{C_{1}}^{2}+q_{7} Q_{C_{7}}+q_{7} Q_{C_{8}} Q_{C_{5}}+q_{5} Q_{C_{2}}^{2} Q_{N_{1}}\right)$ and $Q_{C_{10}}:=q_{7} Q_{C_{8}} Q_{C_{6}}$.

So from 81), 82, , 86) and (85) we get

$$
\left\|C^{m}-C^{n}\right\|_{\mathfrak{H}^{1,2}}^{2} \leq Q_{C_{11}}\left\|C^{m-1}-C^{n-1}\right\|_{L^{2}(\Omega)}^{2}+Q_{C_{12}}\left\|C^{m-1}-C^{n-1}\right\|_{\mathbb{L}_{\Omega, T}}
$$

where

$Q_{C_{11}}:=T e^{T} Q_{C_{3}}+\left(Q_{C_{8}}+1\right) Q_{C_{5}}+Q_{C_{7}}+Q_{C_{9}}$ and $Q_{C_{12}}:=T e^{T} Q_{C_{4}}+Q_{C_{6}}\left(Q_{C_{8}}+1\right)+Q_{C_{10}}$.

Now taking $\mathbb{E}_{\varepsilon}$-expectation we get

$$
\left\|C^{m}-C^{n}\right\|_{\mathbb{Z}}^{2} \leq Q_{C_{11}}\left\|C^{m-1}-C^{n-1}\right\|_{\mathbb{L}_{\Sigma}}^{2}+Q_{C_{12}}\left\|C^{m-1}-C^{n-1}\right\|_{\mathbb{L}_{\Sigma}}
$$

Thus if $\left(C^{m}\right)_{m}$ is Cauchy in $\mathbb{L}_{\Sigma}$ then $\left(C^{m}\right)_{m}$ is also Cauchy in $\mathbb{Z}$, since $Q_{C_{9}}$ and $Q_{C_{10}}$ are bounded for any fixed time $T$.

Thereby, it is sufficient to find a condition for the sequence $\left(C^{m}\right)_{m}$ to be Cauchy in $\mathbb{L}_{\Sigma}$. 
Theorem 3.20 (CPDM time condition and existence). Let $\hat{T}>0$ satisfy the condition $(52)$ then there exists $0<\tilde{T}<\infty$ and $T>0$ satisfying

$$
\left(\mathcal{T}_{C P D M}\right)\left\{\begin{array}{l}
\tilde{T} e^{\tilde{T}}<\frac{1}{\max \left\{Q_{C_{3}} 12\left(C_{R_{3}}^{2}+C_{C}^{2}\right), Q_{C_{4}}\right\}}, \\
T=\min (\hat{T}, \tilde{T}) .
\end{array}\right.
$$

be such that (CPDM) has an unique solution.

Proof. From (81) we see that

$$
\begin{aligned}
\left\|C^{m}-C^{n}\right\|_{\mathbb{L}_{\Omega, T}}^{2} & \leq \tilde{T} e^{\tilde{T}}\left(Q_{C_{3}}\left\|C^{m-1}-C n-1\right\|_{\mathbb{L}_{\Omega, T}}+Q_{C_{4}}\right)\left\|C^{m-1}-C^{n-1}\right\|_{\mathbb{L}_{\Omega, T}} \\
& \leq \tilde{T} e^{\tilde{T}} \max \left\{Q_{C_{3}}\left\|C^{m-1}\right\|_{\mathbb{L}_{\Omega, T}}+\left\|C^{n-1}\right\|_{\mathbb{L}_{\Omega, T}}, Q_{C_{4}}\right\}\left\|C^{m-1}-C^{n-1}\right\|_{\mathbb{L}_{\Omega, T}} .
\end{aligned}
$$

Using (69) and the uniform boundedness of $R_{3}$ (see $(49)$ ) and $C_{0}$ we get

$$
\left\|C^{m}-C^{n}\right\|_{\mathbb{L}_{\Omega, T}}^{2} \leq \tilde{T} e^{\tilde{T}} \max \left\{Q_{C_{3}} 2 e^{\tilde{T} / 2} \tilde{T}\left(C_{R_{3}}^{2}+C_{C}^{2}\right), Q_{C_{4}}\right\}\left\|C^{m-1}-C^{n-1}\right\|_{\mathbb{L}_{\Omega, T}}
$$

Thus, we have that for

$$
\tilde{T} e^{\tilde{T}}<\frac{1}{\max \left\{12 Q_{C_{3}}\left(C_{R_{3}}^{2}+C_{C}^{2}\right), Q_{C_{4}}\right\}}
$$

then $\left(C^{m}\right)_{m}$ is a Cauchy sequence in $\mathbb{L}_{\Sigma}$. So, due to completeness of $\mathbb{L}_{\Sigma}$ we get that $\left(C^{m}\right)_{m}$ converges to $C$ in $\mathbb{L}_{\Sigma}$. From Lemma 3.19 we in turn have that $\left(C^{m}\right)_{m}$ converges to $C$ in $\mathbb{Z}$. Now by invoking Lemma 3.18 we get that $\mathbb{N}^{m}$ converges to $N$ in $\mathbb{X}$.

Furthermore, we get that $\left(C^{m}\right)_{m}(\varsigma)$ and $\mathbb{N}^{m}(\varsigma)$ are Cauchy sequences in $L^{2}\left(0, T ; L^{2}(\Omega)\right)$. So $\left(C^{m}\right)_{m}(\varsigma)$ and $\mathbb{N}^{m}(\varsigma)$ converge to $C(\varsigma)$ and $\mathbb{N}(\varsigma)$ in $L^{2}\left(0, T ; L^{2}(\Omega)\right)$ respectively. In particular, for $\left(C^{m}\right)_{m}(\varsigma)$ due to 79 we get that sequence also converges in $\mathfrak{H}^{1,2}$. Thus the vector $(C(\varsigma), N(\varsigma))$ solves the macroscopic model in a weak sense for a.e. $\varsigma \in \Sigma_{\epsilon}$. Moreover, the solution is unique as proven below.

Theorem 3.21 (Uniqueness). $(C, N) \in \mathbb{Z} \times \mathbb{X}$ is the unique solution to (CPDM).

Proof. Let $N_{1}$ and $N_{2}$ be two solutions to (9c), then it holds that

$$
\int_{\Omega} \partial_{t}\left(N_{1}-N_{2}\right) v d x=\int_{\Omega} R_{4}\left(C_{1}, N_{1}\right)-R_{4}\left(C_{2}, N_{2}\right) v d s
$$

Letting $v:=N_{1}-N_{2}$ and using the Lipschitz continuity of $R_{4}$ we get

$$
\begin{aligned}
\frac{d}{d t}\left\|N_{1}-N_{2}\right\|_{L^{2}(\Omega)}^{2} & \leq 2 C_{\Lambda_{2}}\left(K_{C}\left\|N_{1}-N_{2}\right\|_{L^{2}(\Omega)}^{2}+K_{N} \epsilon\left\|C_{1}-C_{2}\right\|_{L^{2}(\Omega)}^{2}+\frac{\left\|N_{1}-N_{2}\right\|_{L^{2}(\Omega)}^{2}}{\epsilon}\right) \\
& \leq 2 C_{\Lambda_{2}}\left(K_{C}\left\|N_{1}-N_{2}\right\|_{L^{2}(\Omega)}^{2}+\frac{2 K_{N}\left\|N_{1}-N_{2}\right\|^{2}}{\epsilon}\right) \\
& =2 C_{\Lambda_{2}}\left(K_{C}+\frac{2 K_{N}}{\epsilon}\right)\left\|N_{1}-N_{2}\right\|_{L^{2}(\Omega)}^{2}
\end{aligned}
$$

This implies that

$$
\begin{gathered}
\left\|N_{1}-N_{2}\right\|_{L^{2}(\Omega)}^{2} \leq \overbrace{\left\|N_{1}(0)-N_{2}(0)\right\|_{L^{2}(\Omega)}^{2}}^{0} \exp \left(2 C_{\Lambda_{2}} \omega_{2}\left(K_{C}+\frac{2 K_{N}}{\epsilon}\right) t\right) \\
\Rightarrow\left\|N_{1}-N_{2}\right\|_{\mathbb{L}_{\Omega, T}}^{2} \leq 0 \quad \frac{\sqrt[3.18]{\overline{7}}\left\|N_{1}-N_{2}\right\|_{\mathfrak{H}^{1,0}}^{2} \leq 0 \quad \Rightarrow\left\|N_{1}-N_{2}\right\|_{\mathbb{X}}^{2} \leq 0 .}{\sqrt{92}}
\end{gathered}
$$

Hence $N_{1}, N_{2} \in \mathbb{X}$ are a.s. identical.

Now for the uniqueness of $C \in \mathbb{Y}$, let $C_{1}, C_{2} \in \mathbb{Y}$ be such that almost every sample path is a weak solution to $9 \mathrm{~b}$. Also, let $D_{i}:=D\left(C_{i}, N, Y\right)$ and $R_{3}^{i}:=R_{3}\left(C_{i}, N, X\right), i \in\{1,2\}$, where 
$X \in \mathbb{X}, N \in \mathbb{X}$ and $Y \in \mathbb{Y}$ are unique weak solutions to (8a), (9c) and (8b) respectively. Then we have that

$$
\begin{aligned}
\int_{\Omega} \partial_{t}\left(C_{1}-C_{2}\right) v d x & \left.=\int_{\Omega}\left(R_{3}^{1}-R_{3}^{2}\right) v d x-\int_{\Omega}\left(D_{1} \nabla C_{1}\right)-D_{2} \nabla C_{2}\right) \cdot \nabla v d x \\
\frac{1}{2} \frac{d}{d t}\left\|C_{1}-C_{2}\right\|_{L^{2}(\Omega)}^{2} & =\int_{\Omega}\left(R_{3}^{1}-R_{3}^{2}\right)\left(C_{1}-C_{2}\right) d x-\int_{\Omega}\left(D_{1} \nabla C_{1}-D_{2} \nabla C_{2}\right) \cdot \nabla\left(C_{1}-C_{2}\right) d x \\
\leq & \left\|R_{3}^{1}-R_{3}^{2}\right\|_{L^{2}(\Omega)}\left\|C_{1}-C_{2}\right\|_{L^{2}(\Omega)}-c_{D}\left\|\nabla C_{1}-C_{2}\right\|_{L^{2}(\Omega)}^{2} \\
& \quad\left\|D_{1}-D_{2}\right\|_{L^{2}(\Omega)}\left\|\nabla C_{1}\right\|_{H^{1}(\Omega)}^{2}\left\|\nabla C_{1}-\nabla C_{2}\right\|_{H^{1}(\Omega)}^{2} \\
& \leq\left\|R_{3}^{1}-R_{3}^{2}\right\|_{L^{2}(\Omega)}\left\|C_{1}-C_{2}\right\|_{L^{2}(\Omega)}+\left\|D_{1}-D_{2}\right\|_{L^{2}(\Omega)}\left\|\nabla C_{1}\right\|_{H^{1}(\Omega)}^{2}\left\|\nabla C_{1}-\nabla C_{2}\right\|_{H^{1}(\Omega)}^{2} \\
& \leq \frac{1}{\epsilon_{1}}\left\|C_{1}-C_{2}\right\|_{L^{2}(\Omega)}^{2}+\frac{1}{\epsilon_{2}}\left\|D_{1}-D_{2}\right\|_{L^{2}(\Omega)}^{2} \\
& +\epsilon_{1} \underbrace{\left\|R_{3}^{1}-R_{3}^{2}\right\|_{L^{2}(\Omega)}^{2}}_{\text {uniformly bounded }}+\underbrace{\left\|\nabla C_{1}\right\|_{H^{1}(\Omega)}^{4}\left\|\nabla C_{1}-\nabla C_{2}\right\|_{H^{1}(\Omega)}^{4}}_{\text {uniformly bounded }} .
\end{aligned}
$$

So for $\epsilon_{1}, \epsilon_{2}>0$ sufficiently small we get

$$
\begin{aligned}
\frac{1}{2} \frac{d}{d t}\left\|C_{1}-C_{2}\right\|_{L^{2}(\Omega)}^{2} & \leq \frac{1}{\epsilon_{1}}\left\|C_{1}-C_{2}\right\|_{L^{2}(\Omega)}^{2}+\frac{C_{N}}{\epsilon_{2}}\left\|C_{1}-C_{2}\right\|_{L^{2}(\Omega)}^{2}+\frac{C_{C}}{\epsilon_{2}} \underbrace{\left\|N_{1}-N_{2}\right\|_{L^{2}(\Omega)}^{2}}_{=0} \\
& \leq\left(\frac{1}{\epsilon_{1}}+\frac{C_{N}}{\epsilon_{2}}\right)\left\|C_{1}-C_{2}\right\|_{L^{2}(\Omega)}^{2} \\
\Rightarrow\left\|C_{1}-C_{2}\right\|_{L^{2}(\Omega)}^{2} & \leq 0 \Rightarrow\left\|C_{1}-C_{2}\right\|_{\mathbb{L}_{\Omega, T}}^{2} \leq 0 \Rightarrow\left\|C_{1}-C_{2}\right\|_{\mathbb{L}_{\Sigma}}^{2} \leq 0 .
\end{aligned}
$$

Hence $C_{1}, C_{2} \in \mathbb{Z}$ are a.s. identical in $\mathbb{L}_{\Sigma}$. They are also a.s. identical in $\mathbb{Z}$ due to Lemma 3.19 and since $\mathbb{Z} \subset \mathbb{L}_{\Sigma}$

\subsubsection{Measurability of the solution to (CPDM)}

The proof goes similarly to the measurablity proof for the solution of (SPDM).

\subsection{Local and global existence for the solution to the multiscale model}

Theorem 3.22. Local solution: From the existence and uniqueness theorem for (SPDM) (3.10) and (CPDM) (3.20) and the measurability lemmas we get that for the time interval $[0, T)$ with $T>0$ and satisfying the condition $\mathcal{T}_{\mathcal{C P D M}}$ (see $(89)$ ) there exists a unique solution

$$
W=\left(H_{i}, H_{e}, C, N\right) \in \mathbb{X} \times \mathbb{Y} \times \mathbb{Z} \times \mathbb{X}
$$

to the full stochastic multiscale system.

Theorem 3.23. Global solution: For any finite time $\mathbb{T}$ and $0<\epsilon \ll 1$, we can apply the local existence and uniqueness theorem for the intervals $[0, T),[T-\epsilon, 2 T) \ldots[\mathbb{T}-T-\epsilon, \mathbb{T})$.

Proof. In order to apply the local existence proof for the new time interval $[T-\epsilon, 2 T)$, the functions $H_{e}(\varsigma), C(\varsigma)$, and $D(C, N)$ need to be in $W(p, T, \Omega)$, so that $H_{e}(T-\epsilon, \varsigma)$ and $C(T-\epsilon, \varsigma)$, the initial conditions for the new time interval, are in $W^{2, p}(\Omega)$. This can be achieved in the following way. Repeating the argument of Remarks 3 and 4 we get that

$\left(H_{e}^{m}\right)_{m \in \mathbb{N}} \in W(p, T, \Omega)$ and $\left(C^{m}\right)_{m \in \mathbb{N}} \in W(p, T, \Omega)$ are bounded sequences. Also, due to the reflexivity of $W(p, T, \Omega)$ there exists a weakly convergent subsequence $\left(H_{e}^{m_{j}}\right)_{j \in \mathbb{N}} \subset\left(H_{e}^{m}\right)_{m \in \mathbb{N}}$ and $\left(C^{m_{j}}\right)_{j \in \mathbb{N}} \subset\left(C^{m}\right)_{m \in \mathbb{N}}$ such that

$$
H_{e}^{m_{j}} \rightarrow H_{e} \in W(p, T, \Omega) \text { and } C^{m_{j}} \rightarrow C \in W(p, T, \Omega) .
$$

Now, we claim that for a.e. $t \in(0, T)$ and a.e. $\varsigma \in \Sigma_{\epsilon}, H_{e}(t, \varsigma)$ and $C(t, \varsigma)$ solve the equations

$$
\int_{\Omega} \partial_{t} H_{e} \phi d x-\int_{\Omega} \nabla H_{e} \cdot \nabla \phi d x+\int_{\Omega} H_{e} \phi=\int_{\Omega} T\left(H_{i}, H_{e}\right) \phi d x, \quad \forall \phi \in H^{1}(\Omega),
$$


and

$$
\int_{\Omega} \partial_{t} C \phi d x-\int_{\Omega} D(C, N) \nabla C \cdot \nabla \phi d x=\int_{\Omega} R_{3}(C, N) \phi d x, \quad \forall \phi \in H^{1}(\Omega),
$$

respectively.

For $p>d+2$, the above claim can be proved using the following facts and assertions:

1. $W(p, T, \Omega) \hookrightarrow \hookrightarrow L^{p}\left(0, T ; L^{p}(\Omega)\right) \hookrightarrow L^{2}\left(0, T ; L^{2}(\Omega)\right)$,

2. $R_{2}\left(H_{i}^{m}, H_{e}^{m}\right), R_{3}\left(H_{i}, C^{m}, N^{m+1}\right) \in L^{p}\left(0, T ; L^{p}(\Omega)\right)$,

3. $D\left(C^{m}, N^{m+1}\right) \in W(p, T, \Omega)$,

4. $\nabla C^{m} \in L^{p}\left(0, T ; W^{1, p}(\Omega)\right)$ and $\partial_{t} \nabla C^{m} \in L^{2}\left(0, T ;\left(H^{1}(\Omega)\right)^{*}\right)$

5. Assertion 4. and the Lions-Aubin lemma

$\Rightarrow\left\{\phi \in L^{p}\left(0, T ; W^{1, p}(\Omega)\right) ; \phi^{\prime} \in L^{2}\left(0, T ;\left(H^{1}(\Omega)\right)^{*}\right)\right\} \hookrightarrow \hookrightarrow L^{p}\left(0, T ; L^{p}(\Omega)\right)$

Assertion 4. can be shown in the following way:

From (66) we have that

$$
\begin{aligned}
& c_{D}\left(\nabla C_{n}^{m}, \partial_{t} \nabla C_{n}^{m}\right)=\left(R_{3}^{m}, \partial_{t} C_{n}^{m}\right)-\left\langle\partial_{t} C_{n}^{m}, \partial_{t} C_{n}^{m}\right\rangle \\
& c_{D}\left\langle\partial_{t} \nabla C_{n}^{m}, \nabla C_{n}^{m}\right\rangle=\left(R_{3}^{m}, \partial_{t} C_{n}^{m}\right)-\left\langle\partial_{t} C_{n}^{m}, \partial_{t} C_{n}^{m}\right\rangle \\
& c_{D}\left\langle\partial_{t} \nabla C_{n}^{m}, \nabla C_{n}^{m}\right\rangle^{2} \leq 2\left\|R_{3}^{m}\right\|_{L^{2}(\Omega)}^{2}\left\|\partial_{t} C_{n}^{m}\right\|_{L^{2}(\Omega)}^{2}+\frac{d}{d t}\left\|C_{n}^{m}\right\|_{L^{2}(\Omega)}^{4} \\
& \stackrel{\int_{0}^{T}}{\Rightarrow} \int_{0}^{T} c_{D}\left\langle\partial_{t} \nabla C_{n}^{m}, \nabla C_{n}^{m}\right\rangle^{2} d t \leq\left\|R_{3}^{m}\right\|_{L^{2}\left(0, T ; L^{2}(\Omega)\right)}^{4}+\left\|\partial_{t} C_{n}^{m}\right\|_{L^{2}\left(0, T ; L^{2}(\Omega)\right)}^{4}+\left\|C_{n}^{m}\right\|_{L^{2}(\Omega)}^{4}+\left\|C_{0}\right\|_{L^{2}(\Omega)}^{4} \\
& \stackrel{69,700}{\leq} K\left(\left\|R_{3}^{m}\right\|_{L^{2}\left(0, T ; L^{2}(\Omega)\right)}^{4}+\left\|C_{0}\right\|_{L^{2}(\Omega)}^{4}\right)<\infty \text {. }
\end{aligned}
$$

Consider the space

$\mathfrak{W}:=\left\{\phi \in L^{2}\left(0, T ; H^{2}(\Omega)\right) ; \phi^{\prime} \in L^{2}\left(0, T ; L^{2}(\Omega)\right)\right.$ such that $\phi$ solves the equation 46a weakly $\}$.

Due to the existence theorem for 4 (46a) the space $\mathfrak{W} \neq \emptyset$. Also, since one can impose several boundary conditions to $(46 a)$ the set $\mathfrak{W}$ is not a singleton. Clearly, $\mathfrak{W} \subset \mathfrak{H}^{1,2}$.

For all $\phi \in \mathfrak{W}$ its gradient $\phi:=\nabla \phi$ is in $L^{2}\left(0, T ; H^{1}(\Omega)\right)$. So

$$
\int_{0}^{T}\left\langle\partial_{t} \nabla C_{n}^{m}, \tilde{\phi}\right\rangle^{2} d t \leq K\left(\left\|R_{3}^{m}\right\|_{L^{2}\left(0, T ; L^{2}(\Omega)\right)}^{4}+\left\|C_{0}\right\|_{L^{2}(\Omega)}^{4}\right)<\infty .
$$

Since

$$
\mathfrak{W} \subset \mathfrak{H}^{1,2} \subset L^{2}\left(0, T ; H^{1}(\Omega)\right) \Rightarrow L^{2}\left(0, T ;\left(H^{1}(\Omega)\right)^{*}\right) \subset\left(\mathfrak{H}^{1,2}\right)^{*} \subset \mathfrak{W}^{*}
$$

we get that $\partial_{t} \nabla C_{n}^{m} \in L^{2}\left(0, T ;\left(H^{1}(\Omega)\right)^{*}\right)$.

From equation 93 we have that $\left(\partial_{t} \nabla C_{n}^{m}\right)_{n \in \mathbb{N}}$ is an uniformly bounded sequence, hence there exists a subsequence $\left(\partial_{t} \nabla C_{n_{k}}^{m}\right)_{k \in \mathbb{N}} \subset\left(\partial_{t} \nabla C_{n}^{m}\right)_{n \in \mathbb{N}}$ and $v \in L^{2}\left(0, T ;\left(H^{1}(\Omega)\right)^{*}\right)$ such

$$
\partial_{t} \nabla C_{n_{k}}^{m} \stackrel{l \rightarrow \infty}{\longrightarrow} v \text { in } L^{2}\left(0, T ;\left(H^{1}(\Omega)\right)^{*}\right) \text { and }\|v\| \leq \liminf _{k \in \mathbb{N}}\left\|\partial_{t} \nabla C_{n_{k}}^{m}\right\| .
$$

It is clear that the limit $v=\partial_{t} \nabla C^{m}$. Thus assertion 4 . holds.

Using the above five assertions we get that:

$$
\begin{gathered}
C^{m_{j}} \rightarrow C \text { in } W(p, T, \Omega) \Rightarrow C^{m_{j}} \rightarrow C \text { in } L^{p}\left(0, T ; L^{p}(\Omega)\right), \\
H_{e}^{m_{j}} \rightarrow H_{e} \text { in } W(p, T, \Omega) \Rightarrow H_{e}^{m_{j}} \rightarrow H_{e} \text { in } L^{p}\left(0, T ; L^{p}(\Omega)\right), \\
\nabla C^{m_{j}} \rightarrow \nabla C^{m} \text { in } L^{p}\left(0, T ; W^{1, p}(\Omega)\right) \Rightarrow C^{m_{j}} \rightarrow C \text { in } L^{p}\left(0, T ; L^{p}(\Omega)\right) .
\end{gathered}
$$

By dominated convergence and the above Lipschitz estimates for $H_{i}$ and $N$ the claim follows. With $H_{e}, C \in W(p, T, \Omega)$ the local existence and uniqueness proof is applicable to every bounded time sub-intervals of $[0, \mathbb{T})$. 


\section{Numerical simulations}

In this section we present some simulation results for the stochastic multiscale model (abbreviated as SMSM) introduced above. We use a RODE-Taylor scheme 21] for discretizing the intracellular proton equation (1) and implicit finite difference schemes for the rest of the equations.

\subsection{Simulations with a proliferation function switching between growth and decay}

Here we present only the results obtained for SMSM by choosing a $\Lambda_{1}\left(H_{i}\right)$ function that takes both positive and negative values, since other cases are less interesting. Typically, a proliferation function is a nonnegative function influencing the rate of growth. However, as mentioned in Section 2.3, extreme values of $H_{i}$ can induce death even for cancer cells. Since $\Lambda_{1}$ is a function of $H_{i}$ we carry out simulations for the cancer proliferation function $\Lambda_{1}\left(H_{i}\right)$ which is positive for $H_{i}$ in an appropriate interval of the real line.

Figure 4 illustrates the expectation (averaged over 428 sample paths) of the solution to SMSM at different fixed time points. A sequence of increasing time points is chosen to highlight the temporal trend.

Further, in order to easily observe the effects of stochasticity we plot the time evolution graphs at fixed spatial points. To see the spatial trend we choose four equidistantly spaced spatial points. For each of these fixed spatial points, Figures 5 and 6 depict the time evolution of 15 (out of 428) sample paths. In all these figures the average of the sample paths of the solution to (SMSM) is represented by a dashed line.

Figure 5 contains the simulation results for (SMSM) with a Brownian bridge driven noise term. The proliferation function $\Lambda_{1}\left(H_{i}\right)$ is as shown in Figure 3. It is positive for $H_{i}$ approximately in the interval $[0.290785,0.40278]$ and negative for all other positive values of $H_{i}$. Also, it attains its maximum value of 1.4 when $H_{i} \approx 0.3513$. The rate constant $\omega_{1}$ (which we call proliferation sensitivity parameter) was assigned a relatively high value to represent a tumor that is sensitive to changes (positive or negative) induced by $H_{i}$. To contrast this behavior, we performed simulations for a less sensitive (in the above mentioned sense) type of tumor by downscaling the value of $\omega_{1}$ by a factor of 100. The corresponding simulation results are shown in Figure 6 . Altogether, these figures illustrate the following salient features of the (SMSM) model:

1. The (SMSM) model predicts under certain conditions time decay for the cancer cell density $C(t, x)$. This is due to the particular choice of the proliferation function $\Lambda_{1}\left(H_{i}\right)$ which switches between growth and decay. Thus, if the proton dynamics induced by the noise term results in values of $H_{i}$ consistently outside the interval of the positive part of $\Lambda_{1}$ (representing cytotoxicity due to high acidity levels), it then induces a consistent decay in cancer cell density.

2. The observation made in 1 . can be easily verified by inspecting Figure 5 , where some sample paths $C(t,$.$) (cancer cell density) decay to zero and stay there, while some approach$ zero and after a while increase (or start to increase) towards the value 1.

3. From Figure 5 we observe that though some of the sample paths of $C(t, x)$ show consistent decay, its expected behavior (averaged over 428 sample paths ) shows indeed a very slow invasive behavior. The rate of invasion is drastically slowed down due to the decay of $C$ in a certain time span. Another crucial insight that comes to light when Figure 6 is compared with Figure 5 is that a smaller proliferation sensitivity parameter $\left(\omega_{1}\right)$ actually results in higher expected density of cancer cells. This might seem counterintuitive, but it makes sense, as the reduction of $\omega_{1}$ diminishes the effect of the negative values of the switching function; thereby less sample paths undergo decay and as a result the expected density of cancer cells is relatively high.

4. The effect of a switching type of $\Lambda_{1}\left(H_{i}\right)$ function on normal cells is that their apparent extinction time is delayed significantly. 
Figure 4: Plots for expected values of cell densities and proton concentration over the spatial interval $[0,1.9]$ corresponding to different fixed time points. $H_{i}$ is represented by a vertical bar (III) curve, $H_{e}$ by a dashed (- - ) curve, $C$ by the continuous (-) curve and $N$ by a dot-dash (--.-) curve.
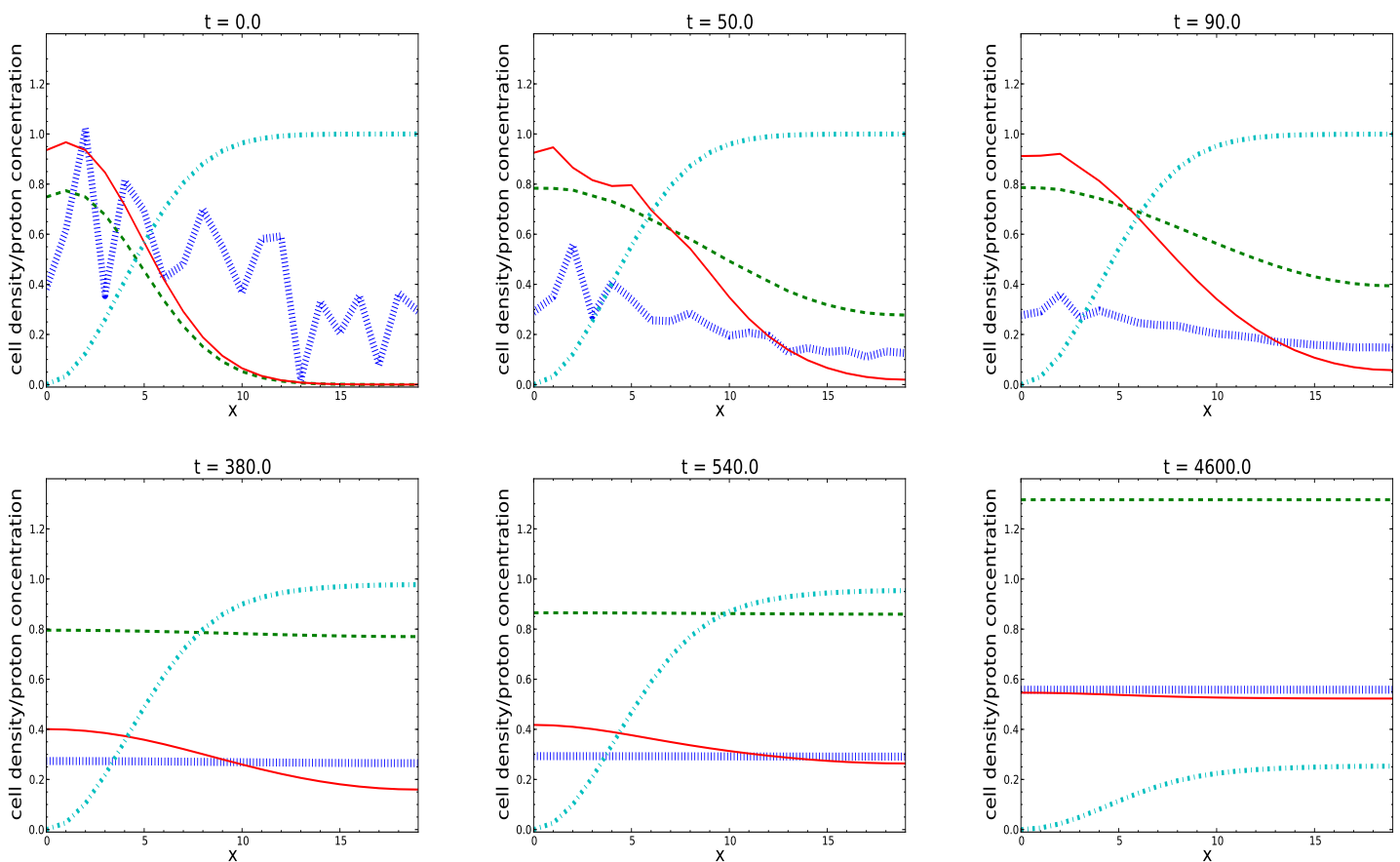

\section{Conclusion}

In this work we proposed and studied a new multiscale model for acid mediated cancer invasion, a topic which has attracted increased attention over the last decade. Since the source and decay terms in our model for the proton dynamics were constructed based on experimental data, the resulting behavior is to some extent reliable. Moreover, the model accounts for the noise and uncertainties at the microscopic level. We proved the wellposedness of the model, thus clearing the way for numerical simulations. From the results we can conclude the following:

1. For a non-negative proliferation function $\Lambda_{1}\left(H_{i}\right)$ (simulations not shown), our stochastic multiscale model predicts sustained tumor invasion. The cancer population density reaches its carrying capacity, the normal cell population density reaches extinction, and its extinction rate is intensified as the cancer cell density approaches its carrying capacity.

2. For a growth function $\Lambda_{1}\left(H_{i}\right)$ taking both positive and negative values, the model exhibits a richer dynamics, especially for the cancer population. If the noise term induces toxic $H_{i}$ values, then $\Lambda_{1}\left(H_{i}\right)$ can become negative, thus leading to a decay in cancer cell density. Hence, the model not only can predict a persistent invasive behavior of cancer cells, but also put in evidence the following interesting features:

- Quiescent and active phases of cancer cells: In the dormant phase, cancer cells are unable to proliferate and are diminishing in number, hence their density may become nearly zero and remain that way until the intracellular acidity $\left(H_{i}\right)$ is suitable for proliferation. In the active phase, cancer cells resume their replication cycle due to a conducive intracellular $\mathrm{pH}$ level. Consequently, their cell density begins to increase, which in turn enhances migration, thereby intensifying the decay of normal cells. Because this phase switching is prominent for relatively high values of $\omega_{1}$ we infer that such behavior is typical for cancer cells sensitive to change induced by $H_{i}$. Since for positively induced changes of $H_{i}$ the proliferation sensitivity parameter is a measure of tumor aggressiveness, we hypothesize that aggressive tumors are sensitive to cytotoxic values of $H_{i}$ and they diminish or surge 
quite rapidly. However, their declining trend may be a transient behavior, i.e. just an entry into a quiescent phase, and they are very well capable of resuming their active phase unless completely eradicated.

- Extinction of cancer cells: If the noise term in (SPDM) induces an intracellular dynamics for which $\Lambda_{1}\left(H_{i}\right)$ is negative for sufficiently long time, then the cancer cell population can approach extinction.

The crucial conclusions here are:

1. As cancer is an ecosystem of various cell types, different types of cancer cells exhibit different phases of behavior (for e.g., quiescent phase, active phase, extinction phase, and invasive phase) at different time moments, such that as a colony they are able to compensate individual deficits and achieve the expected invasive behavior.

2. Aggressive cancers ${ }^{4}$ are illusive in behavior, in the sense that they show signs of rapid depletion in a non-conducive environment, but revert to their true invasive nature as soon as the odds are favorable.

Altogether, the model endowed with a noise term $F\left(\chi_{t}, H_{i}\right)$, proliferation function $\Lambda_{1}\left(H_{i}\right)$ switching between phases of growth and decay and a relatively high proliferation sensitivity parameter, can achieve a robust and tight coupling of the macro- and microscales, thereby allowing for a realistic and collaborative ecosystem type dynamics of cancer cells.

Given the fact that cancer migration is a complex process influenced by its proximal environment and several random cellular mechanisms, deterministic models are too idealistic and sometimes fail to make sufficiently reliable predictions, while stochastic models are not only capable of overcoming this gap, but may also bring to light some rare and interesting features.

\section{Appendix}

\subsection{Nondimensionalisation}

Let $\tau:=10^{-7}$ (measured in $\min$ ) be a time normalizing constant and $K_{w}:=10^{-7}$ (measured in $\left.\frac{M v o l}{\text { cells }}\right)$ be the molar concentration of protons in water per cells per vol. Let the dependent variables $H_{i}$ and $H_{e}$ (both measured in $\frac{\text { Mvol }}{\text { cells }}$ ) be represented in a non-dimensional form as $\bar{H}_{i}:=\frac{H_{i}}{K_{w}}, \bar{H}_{e}:=\frac{H_{e}}{K_{w}}$. Similarly, the time variable $t$ (measured in $\min$ ) and spatial variable $x$ (measured in $d i s t^{5}$ are non-dimensionalized as $\bar{t}=\frac{t}{\tau}, \bar{x}=\frac{x}{\sqrt{D_{1} \tau}}$, where $D_{1}$ (measured in $\frac{\text { dist }^{2}}{\mathrm{~min}}$ ) is the apparent diffusion coefficient of extracellular protons.

The non-dimensional formulation can be deduced using the following rescaling relations:

$$
\left.\begin{array}{lll}
\bar{T}_{1}:=\frac{\tau}{K_{w}} T_{1}, & \bar{T}_{2}:=\frac{\tau}{K_{w}} T_{2}, & \bar{T}_{3}:=\frac{\tau}{K_{w}} T_{3}, \\
\bar{S}_{1}:=\frac{\tau}{K_{w}} S_{1}, & \bar{S}_{2}:=\tau S_{2}, & \bar{Q}:=\frac{\tau}{K_{w}} Q, \\
\bar{\Delta}:=D_{1} \tau \Delta, & \frac{\tau}{K_{w}}:=1, & \bar{t}:=\frac{t}{\tau} .
\end{array}\right\}
$$

For the cell population dynamics we have $N, C, K_{N}, K_{C} \propto \frac{\text { cells }}{v o l}, \omega_{1} \propto \frac{1}{s e c}, \gamma \propto \frac{d i s t}{\sec ^{2}}$. So we define the new unitless variables

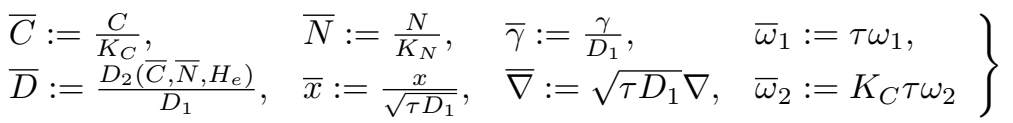

\subsection{Parameters used in the simulations}

We set $\Xi$ (the parameter vector used in the microscopic model) to value $\left(.04,10^{8.5}\right)^{T}$. This choice was made after the stability analysis of (SPDM) without the noise term (i.e., for a deterministic proton dynamics model). The aim of the stability analysis was to choose the parameters so that

\footnotetext{
${ }^{4}$ From the perspective of the proliferation sensitivity parameter $\omega_{1}$ being a measure of aggressiveness of the tumor for positively induced changes by $H_{i}$.

${ }^{5}$ dist refers to some unit of distance suitable for the macroscopic scale of tissues
} 
Table 1: Simulation parameters

\begin{tabular}{|c|c|}
\hline \multicolumn{2}{|c|}{ Noise parameters } \\
\hline$\vartheta$ & $7.3 \times 10^{-5}$ \\
\hline $\mathrm{a}$ & 1 \\
\hline $\mathrm{b}$ & 2 \\
\hline
\end{tabular}

\begin{tabular}{|c|c|}
\hline \multicolumn{2}{|c|}{ Macroscopic parameters } \\
\hline$\omega_{2}$ & .001 \\
\hline$\gamma$ & .001 \\
\hline$\eta_{C}$ & 1 \\
\hline$\eta_{N}$ & .3 \\
\hline
\end{tabular}

\begin{tabular}{|c|c|}
\hline \multicolumn{3}{|c|}{ Numerical parameters } \\
\hline $\mathrm{T}$ (Total time) & 500 \\
\hline $\mathrm{M}$ (\# Monte Carlo simulations) & 428 \\
\hline$\tau$ (Temporal step size) & .1 \\
\hline$h_{x_{1}}$ (Spatial step size along $\left.x_{1}\right)$ & .1 \\
\hline$N_{x_{1}}$ (Grid resolution along $\left.x_{1}\right)$ & 20 \\
\hline
\end{tabular}

Table 2: Parameters for sensitive and less-sensitive type of cancer driven by Brownian Bridge (BB) noise:

\begin{tabular}{|c||c|}
\hline Sensitive: & Less-sensitive: \\
\hline$\omega_{1}= \begin{cases}.5 & \text { if } \Lambda_{1} \geq 0 \\
.05 & \text { if } \Lambda_{1}<0\end{cases}$ & $\omega_{1}= \begin{cases}.005 & \text { if } \Lambda_{1} \geq 0 \\
.0005 & \text { if } \Lambda_{1}<0\end{cases}$ \\
\hline
\end{tabular}

the long term behavior of the deterministic proton model has a stable fixed point whose $H_{i}, H_{e}$ value represents a reverse $\mathrm{pH}$ gradient. Initial conditions were taken as:

$$
\begin{array}{lll}
H_{i}(0, x)=0.4 \exp \left(-\frac{\|x\|_{2}^{2}}{.3}\right)+.7 z(x), & z(x) \sim U([0,1]), & H_{e}(0, x)=0.8 \exp \left(-\frac{\|x\|_{2}^{2}}{3}\right), \\
C(0, x)=\exp \left(-\frac{\|x\|_{2}^{2}}{.3}\right), & N(0, x)=1-\exp \left(-\frac{\|x\|_{2}^{2}}{.3}\right) .
\end{array}
$$


Figure 5: Time evolution plots of SMSM with Brownian bridge noise for 4 different spatial points.

The top left subfigure corresponds to the spatial point $x=0$, the top right to the spatial point

$x=.5$, bottom left to the spatial point $x=1$, and bottom right to the spatial point $x=1.5$.
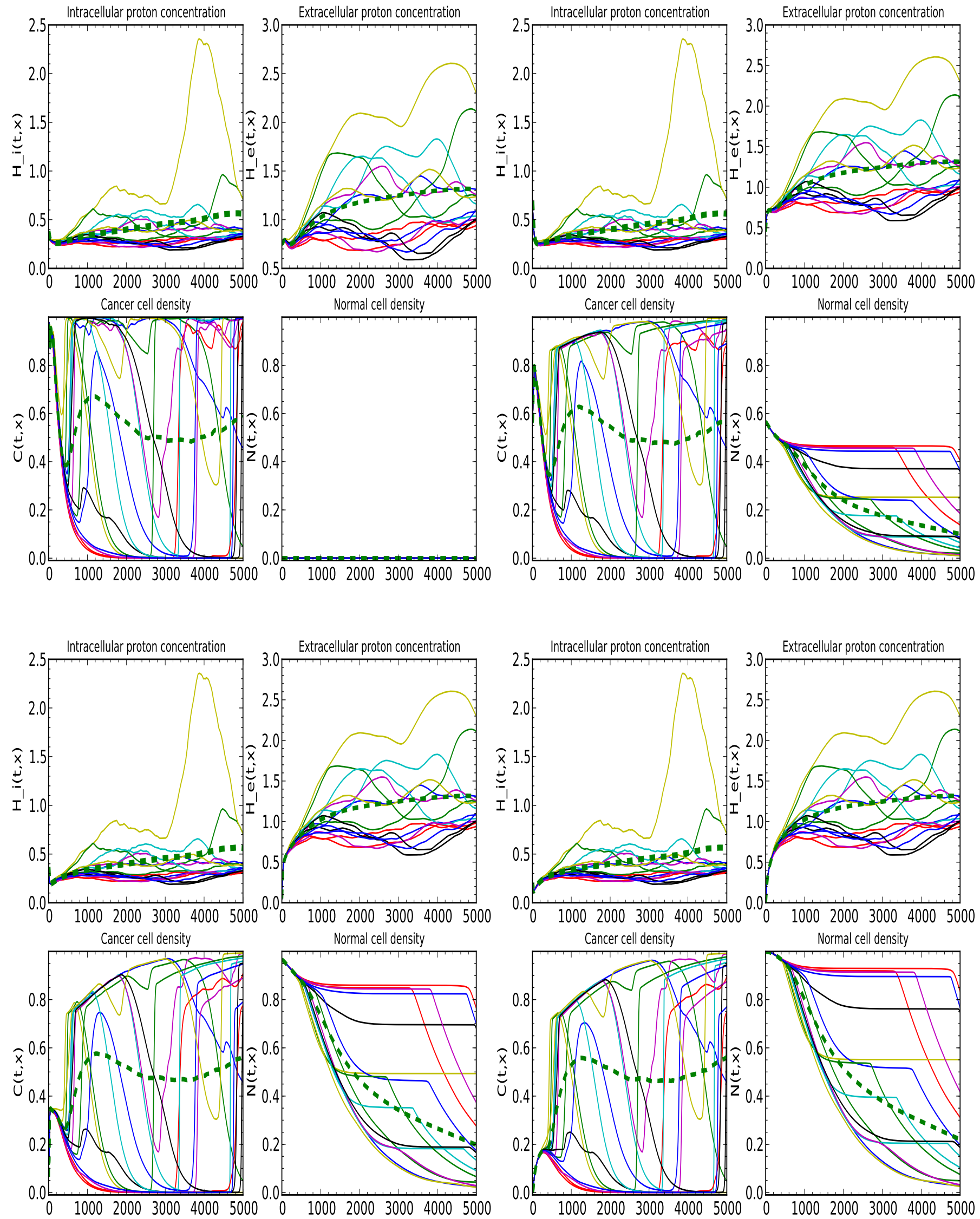
Figure 6: Time evolution plots of SMSM with Brownian bridge noise with small proliferation sensitivity parameter for 4 different spatial points. The top left subfigure corresponds to the spatial point $x=0$, the top right to the spatial point $x=.5$, bottom left to the spatial point $x=1$, and bottom right to the spatial point $x=1.5$.
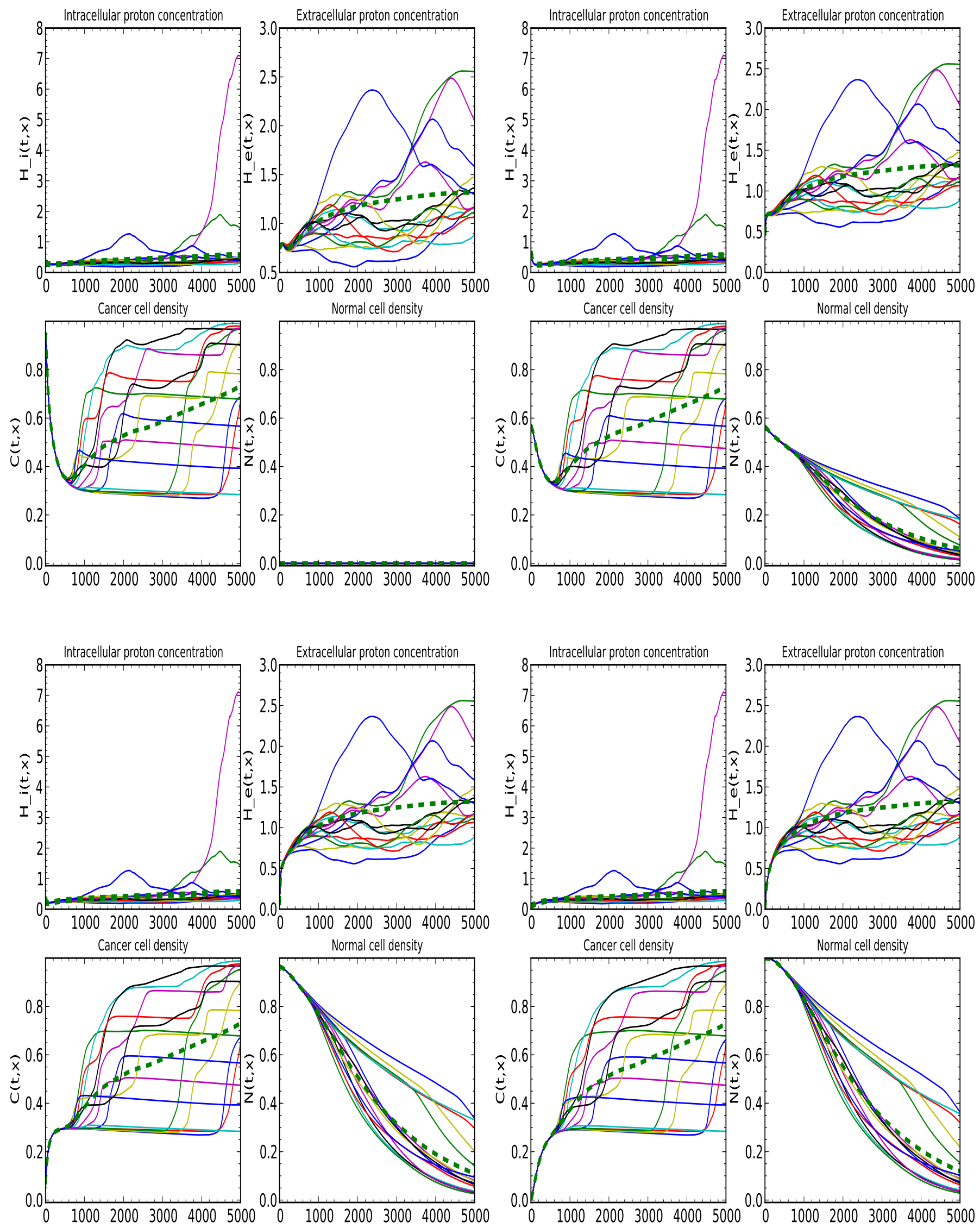


\section{References}

[1] O.R. Abakarova. The metastatic potential of tumors depends on the $\mathrm{pH}$ of host tissues. Bull. Exp. Biol. Med., 120:1227-1229, 1995.

[2] J. Almquist, P. Lang, D. Prätzel-Wolters, J.W. Deitmer, M. Jirstrand, and H.M. Becker. A kinetic model of the monocarboxylate transporter mct1 and its interaction with carbonic anhydrase II. Jounal of computer science and system biology, (3):107-116, 2010.

[3] P. Baluk, H. Hashizume, and D.M. McDonald. Cellular abnormalities of blood vessels as targets in cancer. Current Opinion in Genetics \& Development, 15(1):102-111, 2005.

[4] M.J. Boyer and I.F. Tannock. Regulation of intracellular pH in tumor cell lines: Influence of microenvironmental conditions. Cancer Research, 52(16):4441-4447, 1992.

[5] J.R. Casey, S. Grinstein, and J. Orlowski. Sensors and regulators of intracellular pH. Nature Review Molecular Cell Biology, 11(1):50 - 61, 2010.

[6] L.C. Evans. Partial Differential Equations, volume 49. AMS, 1997.

[7] A. Fasano, M. Herrero, and M. Rocha Rodrigo. Slow and fast invasion waves in a model of acid-mediated tumour growth. Math. Biosci., 220:45-56, 2009.

[8] R.A. Gatenby and E.T. Gawlinski. A reaction-diffusion model of cancer invasion. Cancer Research, 56(24):5745-5753, 1996.

[9] R.A. Gatenby and E.T. Gawlinski. The glycolytic phenotype in carcinogenesis and tumor invasion: Insights through mathematical models. Cancer Research, 63(14):3847-3854, 2003.

[10] R.A. Gatenby, E.T. Gawlinski, A.F. Gmitro, B. Kaylor, and R.J. Gillies. Acid-mediated tumor invasion: a multidisciplinary study. Cancer Research, 66(10):5216-5223, 2006.

[11] R.A. Gatenby and R.J. Gillies. Why do cancers have high aerobic glycolysis? Nature Review Cancer, 4(11):891 - 899, 2004.

[12] R.A. Gatenby and R.J. Gillies. Glycolysis in cancer: A potential target for therapy. The International Journal of Biochemistry and Cell Biology, 39(78):1358 - 1366, 2007.

[13] B. S. Govindan, W. B. Spillman, J. L. Robertson, and W. R. Huckle. Acid-mediated tumor invasion: How does vasculature affect the growth characteristics? 2004. Provided by the SAO/NASA Astrophysics Data System.

[14] D. Hanahan and R.A. Weinberg. Hallmarks of cancer: The next generation. Cell, 144(5):646 $-674,2011$.

[15] S.A. Hiremath. Stochastic multiscale modeling of acid mediated cancer invasion, September 2013. Master thesis.

[16] J.S. Horne, E.O. Garton, S.M. Krone, and J.S. Lewis. Analyzing animal movements using Brownian bridges. PubMed, Ecology, 88(9):2354-63, 2007.

[17] M. Irving, J. Maylie, L.N. Sizto, and K.W. Chandler. Intracellular diffusion in the presence of mobile buffers. application to proton movement in muscle. Biophysical journal, 57(4):717 $-721,1990$.

[18] R.K. Jain. Normalizing tumor vasculature with anti-angiogenic therapy: A new paradigm for combination therapy. Nature Medicine, 7(9):987-989, 2001.

[19] E. Jakobsson and S.W. Chiu. Stochastic theory of ion movement in channels with single-ion occupancy. application to sodium permeation of gramicidin channels. Biophys. J., 52:33-45, 1987.

[20] A. Jentzen and P.E. Kloeden. Pathwise Taylor schemes for random ordinary differential equations. BIT Numerical Mathematics, 49(1):113-140, 2009. 
[21] A. Jentzen and P.E. Kloeden. Taylor Approximations for Stochastic Partial Differential Equations. SIAM, 2011.

[22] W. Junge and S. McLaughlin. The role of fixed and mobile buffers in the kinetics of proton movement. Biochimica et Biophysica Acta (BBA) - Bioenergetics, 890(1):1 - 5, 1987.

[23] J. Kelkel and C. Surulescu. On a stochastic reaction-diffusion system modeling pattern formation on seashells. Journal of Mathematical Biology, 60(6):765-796, 2010.

[24] P.E. Kloeden and E. Platen. Numerical Solution of Stochastic Differential Equations. Springer, 1995.

[25] O. A. Ladyzhenskaya, V. A. Solonnikov, and N. N. Uraltseva. Linear and quasilinear equations of parabolic type, volume 23 of Translations of Mathematical Monographs. American Mathematical Society, Providence, R.I., 1967.

[26] D. Le and H. Smith. Strong positivity of solutions to parabolic and elliptic equations on nonsmooth domains. Journal of Mathematical Analysis and Applications, 275(1):208 - 221, 2002.

[27] A.H. Lee and I.F. Tannock. Heterogeneity of intracellular $\mathrm{pH}$ and of mechanisms that regulate intracellular pH in populations of cultured cells. Cancer Research, 58:1901-1908, 1998.

[28] C. Märkl, G. Meral, and C. Surulescu. Mathematical analysis and numerical simulations for a system modeling acid mediated tumor cell invasion. International Journal of Analysis, 2013:15, 2013

[29] N.K. Martin, E.A. Gaffney, R.A. Gatenby, and P.K. Maini. Tumor-stromal interactions in acid-mediated invasion: A mathematical model. J. Theor. Biol., 267:461-470, 2010.

[30] R. Martinez-Zaguilan, E.A. Seftor, R.E.B. Seftor, Y. Chu, R.J. Gillies, and M.J.C. Hendrix. Acidic pH enhances the invasive behavior of human melanoma cells. Clin. Exp. Metastasis, 14:176-186, 1996.

[31] G. Meral, C. Stinner, and C. Surulescu. On a multiscale model involving cell contractivity and its effects on tumor invasion. preprint, TU Kaiserslautern (2013). Submitted.

[32] G. Meral and C. Surulescu. Mathematical modeling, analysis and numerical simulations for the influence of heat shock proteins on tumor invasion. Journal of Math. Analysis and Applications, 408:597-614, 2013.

[33] E. Racker. Why do tumor cells have a high aerobic glycolysis? Journal of Cellular Physiology, 89(4):697-700, 1976.

[34] A. Roos and W.F. Boron. Intracellular pH. Physiological Reviews, 61(2):296-434, 1981.

[35] L.D. Shrode, H. Tapper, and S. Grinstein. Role of intracellular $\mathrm{pH}$ in proliferation, transformation, and apoptosis. Journal of Bioenergetics and Biomembranes, 29(4):393-399, 1997.

[36] K. Smallbone, D.J. Gavaghan, R.A. Gatenby, and P.K. Maini. The role of acidity in solid tumor growth and invasion. J. of Theor. Biol., 235:476-484, 2005.

[37] C. Stinner, C. Surulescu, and M. Winkler. Global weak solutions in a PDE-ODE system modeling multiscale cancer cell invasion. SIAM J. Math. Analysis, 46:1969-2007, 2014.

[38] C. Stock and A. Schwab. Protons make tumor cells move like clockwork. European J. Physiology, 458:981-992, 2009.

[39] C. Surulescu and N. Surulescu. A nonparametric approach to cell dispersal. Int. J. Biomath. Biostat., 1:109-128, 2010. 
[40] C. Surulescu and N. Surulescu. Modeling and simulation of some cell dispersion problems by a nonparametric method. Math. Biosc. Eng., 8:263-277, 2011.

[41] C. Surulescu and N. Surulescu. Some classes of stochastic differential equations as an alternative modeling approach to biomedical problems,. in P.E. Kloeden and C. Pötzche (eds), Random and Nonautonomous Dynamical Systems in the Life Sciences, LNM 2102, Biomathematics Series, Springer, 2013.

[42] P. Swietach, M. Zaniboni, A.K. Stewart, A. Rossini, K.W. Spitzer, and R.D. Vaughan-Jones. Modelling intracellular $\mathrm{H}^{+}$ion diffusion. Progress in Biophysics and Molecular Biology, 83(2):69 - 100, 2003.

[43] P. Vaupel, F. Kallinowski, and P. Okunieff. Blood flow, oxygen and nutrient supply, and metabolic microenvironment of human tumors: A review. Cancer Research, 49(23):6449-6465, 1989.

[44] B.A. Webb, M. Chimenti, M.P. Jacobson, and D.L. Barber. Dysregulated pH: a perfect storm for cancer progression. Nature Review Cancer, 11(9):671-7, 2011.

[45] S.D. Webb, J.A. Sherratt, and R.G. Fish. Alterations in proteolytic activity at low pH and its association with invasion: a theoretical model. Clin Exp Metastasis, 17(5):397-407, 1999.

[46] S.D. Webb, J.A. Sherratt, and R.G. Fish. Mathematical modelling of tumor acidity: Regulation of intracellular pH. Journal of Theoretical Biology, 196(2):237 - 250, 1999.

[47] M. Yilmaz, G. Christofori, and F. Lehembre. Distinct mechanisms of tumor invasion and metastasis. Trends in Molecular Medicine, 13(12):535 - 541, 2007. 